\title{
WestVirginiaUniversity
}

THE RESEARCH REPOSITORY @ WVU

Graduate Theses, Dissertations, and Problem Reports

2014

\section{Quantifying the Limits of Fingerprint Variability}

\author{
Michael Fagert \\ West Virginia University
}

Follow this and additional works at: https://researchrepository.wvu.edu/etd

\section{Recommended Citation}

Fagert, Michael, "Quantifying the Limits of Fingerprint Variability" (2014). Graduate Theses, Dissertations, and Problem Reports. 327.

https://researchrepository.wvu.edu/etd/327

This Thesis is protected by copyright and/or related rights. It has been brought to you by the The Research Repository @ WVU with permission from the rights-holder(s). You are free to use this Thesis in any way that is permitted by the copyright and related rights legislation that applies to your use. For other uses you must obtain permission from the rights-holder(s) directly, unless additional rights are indicated by a Creative Commons license in the record and/ or on the work itself. This Thesis has been accepted for inclusion in WVU Graduate Theses, Dissertations, and Problem Reports collection by an authorized administrator of The Research Repository @ WVU. For more information, please contact researchrepository@mail.wvu.edu. 


\title{
Quantifying the Limits of Fingerprint Variability
}

\author{
Michael Fagert
}

\author{
Thesis submitted \\ to the College of Eberly Arts and Sciences \\ at West Virginia University \\ in partial fulfillment of the requirements for the degree of \\ Master of Science in \\ Forensic and Investigative Science
}

\author{
Keith Morris, Ph.D., Chair \\ Jacqueline Speir, Ph.D \\ Robert Williams
}

Forensic and Investigative Science Program

\section{Morgantown, West Virginia}

2014

Keywords: fingerprints, minutiae, distortion, variability, quantification, limits, latent, template Copyright 2014 Michael Fagert 


\section{ABSTRACT \\ Quantifying the Limits of Fingerprint Variability}

Michael Fagert

Fingerprints are one of the most widely used identification features in both the biometric and forensic fields. However, the comparison and identification of fingerprints is made difficult by fingerprint variability arising from distortion. This study quantifies the limits of fingerprint variability when subject to heavy distortion, and the variability observed in repeated inked planar impressions. Fingers were video recorded performing several distortion conditions under heavy deposition pressure: left, right, up, and down translation of the finger, clockwise and counterclockwise torque of the finger, and planar impressions. Fingerprint templates, containing 'true' minutiae locations, were then created from 10 inked planar impressions for 30 separate fingers. The 30 fingers studied consisted of 10 right slant loops, 10 plain arches, and 10 plain whorls. A minimal amount of variability, .18 mm globally, was observed for minutiae in inked planar impressions. When subject to heavy distortion minutiae can be displaced by upwards of $3 \mathrm{~mm}$ and their orientation altered by as much as $30^{\circ}$. Minutiae displacements of $1 \mathrm{~mm}$ and $10^{\circ}$ changes in orientation are readily observed. The results of this study will allow fingerprint examiners to identify and understand the degree of variability that can be reasonably expected throughout the various regions of fingerprints. 


\section{Table of Contents}

1. Introduction 1

2. Previous Research 2

3. Materials $\quad 4$

4. Methods 5

Distortion Videos $\quad 6$

Inked Planar Impressions $\quad 7$

Finger Template Generation $\quad 7$

Distorted Images and Image Processing $\quad 8$

Calculating Degree of Variability 9

Distortion Maps $\quad 10$

5. Results 11

Justification of Section Determination $\quad 11$

Examiner Variability $\quad 12$

Variability of Inked Planar Impressions $\quad 12$

Characterization and Directionality of Distortion Types 16

$\begin{array}{ll}\text { Distortion and Pattern Type } & 20\end{array}$

$\begin{array}{ll}\text { Finger Number } & 29\end{array}$

Gender 31

Weight Loss $\quad 32$

Orientation $\quad 32$

Distortion Maps $\quad 35$

Test of Model 41

Practical Applicability $\quad 42$

6. Conclusion $\quad 43$

7. References $\quad 45$

8. Appendix A 46

9. Appendix B $\quad 55$

10. Appendix C 58

11. Appendix D $\quad 65$

12. Appendix E $\quad 72$ 


\section{Introduction}

Fingerprints are one of the most widely used biometric identification features, and are commonly encountered evidence at crime scenes. The comparison and identification of fingerprints can be used to identify persons, place individuals at a particular location, and link cases together. However, comparison of fingerprints is made difficult by a multitude of distortion factors producing variability in fingerprint impressions. Fingerprint variability arises from the elasticity of friction ridge skin and the inability to exactly repeat the conditions in which a fingerprint was deposited. The purpose of this study is to quantify and characterize the limits of fingerprint variability when subjected to heavy distortions.

The results of this study will address concerns raised by The National Academy of Science 2009 report, Strengthening Forensic Science in The United States: A Path Forward, on fingerprint variability and distortion. The report states:

"the impression left by a given finger will differ every time, because of inevitable variations in pressure, which change the degree of contact between each part of the ridge structure and the impression medium. None of these variabilities - of features across a population of fingers or of repeated impressions left by the same finger has been characterized, quantified, or compared"[1].

The report later states, "examiners can too easily explain a 'difference' as an 'acceptable distortion' in order to make an identification" [1]. This study will address these statements by quantifying and characterizing the variability both in repeated impressions of the same finger and across a population of fingers, and by creating distortion maps that examiners can use to explicitly describe 'differences' throughout a fingerprint arising from distortion.

Most fingerprint comparisons are facilitated with the use of automated matching systems. These systems typically employ a minutiae based matching algorithm that first extracts minutiae locations and orientations from both fingerprint images. The algorithm then aligns the two images through a registration that translates, rotates, and scales the images to create the best possible fit [2]. Minutiae are determined to match if they fall within a tolerance zone or bounding box placed around the template image minutiae. However, the elasticity of friction ridge skin produces non-linear distortions which makes a global registration impractical for aligning and matching two fingerprint images [3]. Registration and matching must be considered at both the local and global levels.

To quantify the limits of variability, the locations of minutiae in distorted fingerprint images will be compared to those of minutiae in template 'images'. Template images will be constructed from multiple inked planar impressions of fingers. The templates will consist of the average minutiae locations of each minutiae present in all inked impressions for the finger. Planar impressions can be recorded under controlled conditions and contain minimal distortion from deposition pressure, rolling, lateral movements, and rotation of the finger. The main distortion present in planar impressions arises from the 3D to 2D recording of the fingerprint [3]. This type of distortion can be ignored as it is inevitable in the recording of any fingerprint. 
Distorted images will be isolated from video recordings of fingers performing lateral and rotational movements on a fixed piece of glass. The following movements will be performed under heavy deposition pressure: left, right, up, and down translation, clockwise and counterclockwise torque, and planar glass impressions. These movements seek to simulate actions such as forcing open a window or intentional altering livescan recordings. For the purposes of this study, translation and torque will refer to the movement of the finger itself and not the features of the fingerprint. Each movement will be performed up to, but not past the point of finger slippage. The locations of the minutiae in the distorted images will then be compared to those in their respective templates.

\section{Previous Research}

Most of the research on fingerprint distortion and variability has been dedicated to making automated matching systems more robust to non-linear distortions and better suited to align fingerprint images. This is accomplished by adjusting bounding boxes, developing new matching algorithms, attempting to model distortion, or attempting to remove distortion in fingerprints $[2,3,4,5,6,7]$. The desired result of these studies and algorithms is to lower both the false acceptance and false rejection rates of the matching system.

One of the earlier studies on modeling fingerprint distortion was conducted in 2001 by Cappelli et al. Cappelli [3] studied distortions arising in on-line sensors and noted three distinct fingerprint regions: close contact region, transitional region, and external region (Figure 1).

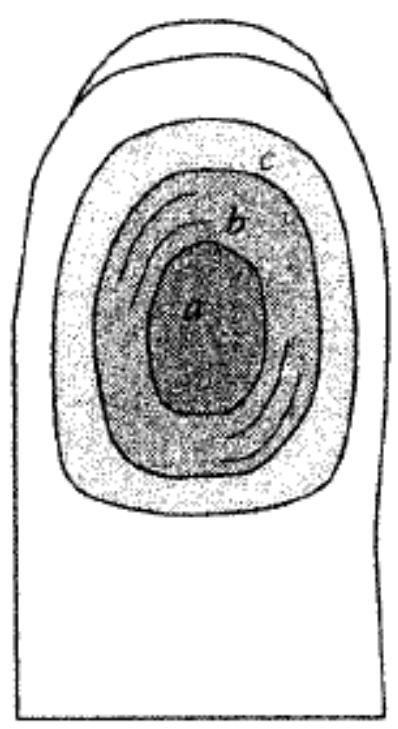

Figure 1. The close contact region (a), transitional region (b), and external region (c) of a finger in contact with an on-line sensor.

High pressure in the close contact region prevents slippage and minutiae remain static, a looser contact in the transitional region results in non-linear distortions, and light contact in the external region results in a rigid, linear distortion [4]. As the pressure between the finger and surface decreases, the amount of possible distortion increases. This defines a radial property for distortion and fingerprint variability. The rigid distortion in the external region is modeled by a 
full affine transformation (using translation and rotation of features without scalar transformation).

To account for the varying amount distortion in the transitional region, a fractional amount of the affine transformation is applied throughout the region. A small amount of the transformation is applied to the areas close to the center of the print, while a large amount of the transformation is applied to the edges of the transitional region. An increasing amount of affine transformation is applied to mimic the radial property of distortion. As a result, the distorted and template prints are registered to fit more closely at both the local and global levels.

The method proposed in [3] produced improved matching capabilities when tested against a test database, but affine transformations are still a rigid transformation. A thin-plate spline approach was proposed by Bazen and Gerez [2] which elastically models distortion throughout a fingerprint. Thin-plate splines are virtual metal plates that can normalize two images based on the bending energy of the plate at specific landmark points. In the proposed method, the splines are fixed above the fingerprint images at a set of minutiae (landmark points). The amount of bending energy at each point is determined by how much the minutiae has been displaced in relation to the template image; the larger the displacement the larger the bending energy.

Based upon the bending energy at the landmark points the thin-plate splines interpolate the amount of bending energy throughout the fingerprint, and elastically transforms the distorted image to resemble the template [2]. The end result of this method is a better fit of both ridges and minutiae throughout the entire fingerprint. A better global fit lowers the possibility of false rejections, and allows the matching system to employ smaller bounding boxes to prevent false acceptances.

As stated previously, automated systems deem two minutiae to match if the location of each falls within a set bounding box. Most systems employ bounding boxes of a fixed radius or dimensions throughout the fingerprint. The size of the bounding boxes can be increased to make the system more robust to non-linear distortions, but this can have a direct increase in the false acceptance rate of minutiae pairs. Recently, some studies have implemented polar coordinate based bounding boxes into their minutiae algorithms.

According to Jain [5] and He [6], a polar coordinate system is better suited to account for the radial property of distortion. Jain implements a bounding box with a fixed radius and radial angle range throughout the finger. While these values remain fixed, a larger area will be covered by bounding boxes further from the center of the finger. He employs a variable sized bounding box system to further the robustness of their matching algorithm. The two systems can be seen in Figure 2. The variable size bounding systems uses a larger radial angle for minutiae closer to the center of the finger. The reasoning for a larger radial angle is that a small deformation close to the center of the finger will produce a larger radial angle change than a displacement of equal magnitude far from the center of the finger. 

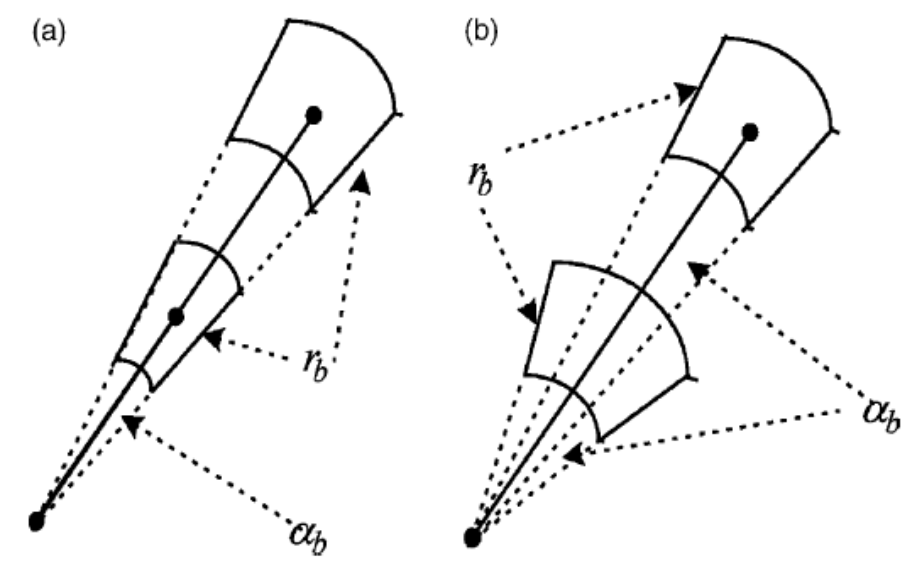

Figure 2. Fixed bounding boxes applied by Jain (a), and variable sized boxes applied by He (b)

Further studies seek to remove distortion by normalizing a distorted image in relation to a known template image. The goal of these studies is to generate a better fit between both the minutiae and the ridges of the distorted and template images much like the thin-plate spline models. Studies attempting to remove distortion typically operate on the idea that there is consistent ridge spacing throughout a fingerprint. Senior and Bolle [7] have had success normalizing two prints by determining the average ridge spacing in a distorted print and applying it throughout the entire image.

While no studies are known to quantitatively describe distortion in fingerprints, a study was found that qualitatively describes fingerprint deformations [8]. Maceo studied two index fingers: a loop and a whorl. Each finger was placed on a piece of glass with various deposition pressures and videotaped performing different movements. The fingers were moved left, right, proximally, distally, and torqued counterclockwise and clockwise. The distance moved by each finger before slippage occurred was measured for each of the movements.

Maceo noted that areas with a large amount of parallel flowing ridges had a greater potential for flexibility. Furrows on the leading edge of the finger were observed to expand while the furrows on the trailing edge of the finger compressed. This means that when the finger was moved distally, the furrows above the core area expanded and the furrows below the core area compressed. Both fingers were also able to move the most under heavy deposition pressure before slipping. The fingers moved nearly $3 \mathrm{~mm}$ under distal movements, and approximately 1 $\mathrm{mm}$ for the other movements. The two fingers could be rotated almost 30 degrees before slipping under clockwise and counterclockwise torque. While informative and providing some characterization of distortions, the study involved a limited sample size and provided little detail about how much the minutiae themselves were affected by each movement.

\section{Materials}

- Black fingerprint ink (Evident ${ }^{\circledR}$ Crime Scene Products)

- Ink roller (Evident ${ }^{\circledR}$ Crime Scene Products)

- Ink Plate 
- White cardstock paper

- Nikon $^{\circledR} \mathrm{D} 7100^{\mathrm{TM}}$ camera

- Triple Axis Bubble Level: Flash Shoe Mount (Polaroid)

- Nikon $^{\circledR}$ AF Micro Nikkor $60 \mathrm{~mm}$ f/2.8D Lens

- SanDisk Ultra ${ }^{\circledR}$ CompactFlash $^{\circledR}$ (8GB)

- Quadra-Pod ${ }^{\mathrm{TM}}$ (or tripod)

- KimTech Science ${ }^{\circledR}$ Kim Wipes ${ }^{\circledR}$

- LabPro PowerLite (Dolan-Jenner Industries)

- 100 W Soft Incandescent Light Bulb with Clamp

- Plane Polarizing Sheet

- $\quad$ 1TB Seagate ${ }^{\circledR}$ Expansion Drive

- ImageJ

- CLAHE plugin

- Flatbed Scanner

- NIST 6 inch ruler

- Nikon $^{\circledR}$ ViewNX2 ${ }^{\mathrm{TM}}$

- 8" x 10" Glass, 1/8" thick (6)

- 42" two-by-four (4)

- 15.5 " x 36 " oak plywood, $1 / 2$ " thick

- 2x4 Basics shelf link kit

- Glass clips (6)

- $3 / 4 "$ Screws (6)

- Cordless drill

\section{Methods}

A total of 30 fingers from 27 subjects were analyzed in this study. The fingers consisted of 10 right slant loops, 10 plain whorls, and 10 plain arches. The subjects were comprised of 16 males and 11 females between the ages of 18 and 30. A single finger was used from each subject except in the case of arches. Only 7 individuals with plain arch pattern types were readily available so three fingers were use from a male and two fingers from a female subject. The 30 fingers studied consisted of 21 index fingers, 5 thumbs, 2 middle fingers, and 2 ring fingers. 
Each finger was video recorded performing distortional movements, photographed, and multiple inked impressions were recorded.

\section{Distortion Videos}

A basic apparatus was constructed for the purpose of collecting distortion videos. A $2 \times 4$ Basics shelf links kit was used to construct a table with four $2 \times 4 \mathrm{~s}$ and a 15.5" 336 " piece of oak plywood. A 6" 8 " hole was cut in the center of the plywood and an 8 " x 10 " piece of glass was fixed above the hole with 6 glass clips. The apparatus can be seen in Figure 3. A Nikon ${ }^{\circledR}$
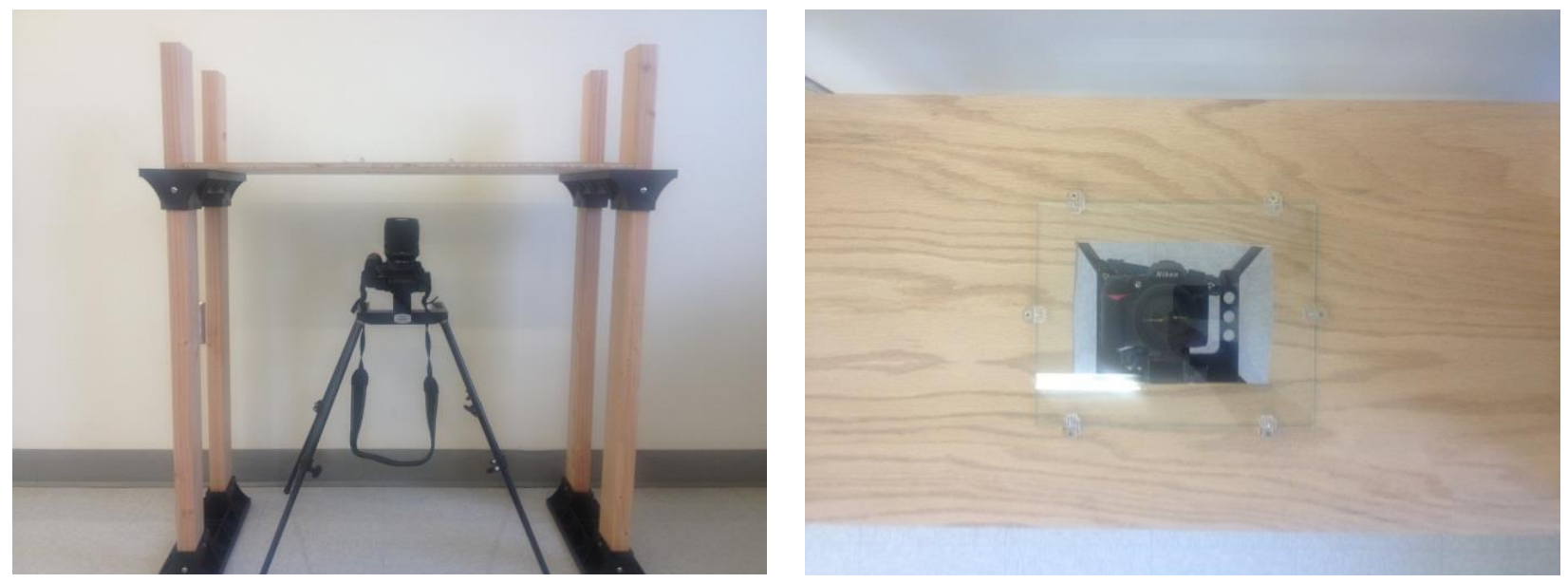

Figure 3. Shelving apparatus constructed for distortion videos with piece of glass fixed over a hole cut in the center of the plywood shelf piece.

D7100 ${ }^{\mathrm{TM}}$ camera, equipped with a Nikkor ${ }^{\circledR} 60 \mathrm{~mm} \mathrm{f} / 2.8 \mathrm{D}$ lens, was mounted on a Quadra-Pod ${ }^{\mathrm{TM}}$ beneath the piece of glass to recorded the videos. A Labpro PowerLite and 100W soft incandescent light bulb were used to obliquely light the finger as the videos were recorded. A plane polarizing sheet was placed in front of the incandescent light bulb, providing the best lighting conditions.

Distortion videos were recorded first so that no ink was present on the finger, and the skin would not be irritated from scrubbing ink off the finger. The following distortional movements were recorded for each individual: right, left, up, and down translation of the finger, clockwise and counter-clockwise torque of the finger, and planar glass impressions. Before recording the videos, the various movements were explained verbally and demonstrated for the subject. Emphasis was placed upon not allowing the finger to slide across the glass and not to roll the finger. Subjects were then allowed to practice each movement on the glass.

The subjects were then asked to wipe any dirt or debris off their finger and the glass was wiped clean with a Kim Wipe ${ }^{\circledR}$. Once the finger and glass were cleaned, the subject placed their finger down firmly on the glass above the camera. A NIST six inch ruler was placed facing downward beside the finger, and the subject performed each distortional movement 7 times. If the subject's finger slipped or was rolled during the video process, the video was re-recorded. 


\section{Inked Planar Impressions}

Each finger was then photographed before recording inked planar impressions. This was done for two reasons: to observe the natural appearance of the friction skin and pattern with no distortion, and to be sure ending ridges were recorded as ending ridges and bifurcations as bifurcations in the inked impressions. A small amount of Evident ${ }^{\Theta}$ black fingerprint ink was deposited on a metal ink plate and dispersed with a standard ink roller.

The subject's finger was then rolled in the ink to ensure complete inking of the finger, and a planar impression was placed on a piece of white cardstock. The finger was lightly reinked and another planar impression was placed onto the cardstock. This process was repeated until at least 10 clear and complete planar impressions were recorded. The inked impressions were scanned onto an external hard drive at 1000 ppi. The same NIST ruler used for the distortion videos was included in the scanned image of the inked planars to set an accurate scale in the scanned images.

\section{Finger Template Generation}

The scanned image of the inked planar impressions for each finger was opened in ImageJ. A line, $10 \mathrm{~mm}$ in length, was drawn on the NIST ruler with the straight line tool. Under the Analyze tab, this length was indicated and the scale for the image was set to $\mathrm{mm}$. The 10 best inked impressions for each finger were then isolated.

The minutiae present in all of the 10 planar impressions were then marked with the multipoint selector tool. Ending ridges were marked in the center of the ridge at its ending point. Bifurcations were marked in the middle of the area where the initial ridge divides into two separate ridges. An example of the minutiae marking for inked impressions is shown in Figure 4 below. To ensure consistent marking of each minutiae, the minutiae were marked in the first impression, and this image was used to as a guide to mark the remaining nine images. Under the Analysis tab, the Measure feature was used to extract the $\mathrm{x}$ and $\mathrm{y}$ coordinates of all the minutiae marked in the images. These coordinates were uploaded into an Excel spreadsheet.

ImageJ automatically assigns an origin point for all images in the top left corner of the image. A minutiae or feature was selected from the core area of each finger to serve as the new origin of the image. This location was selected based on the close contact region proposed by [3] where little to no distortion occurs in the finger. The $\mathrm{x}$ and $\mathrm{y}$ coordinates of this feature were subtracted from those of the entire minutiae set to zero them in respect to the new origin. Because the origin set by ImageJ is in the top left corner of the image, all y coordinates will initially be negative. To account for this, all y coordinates are multiplied by negative 1 before they are zeroed. 


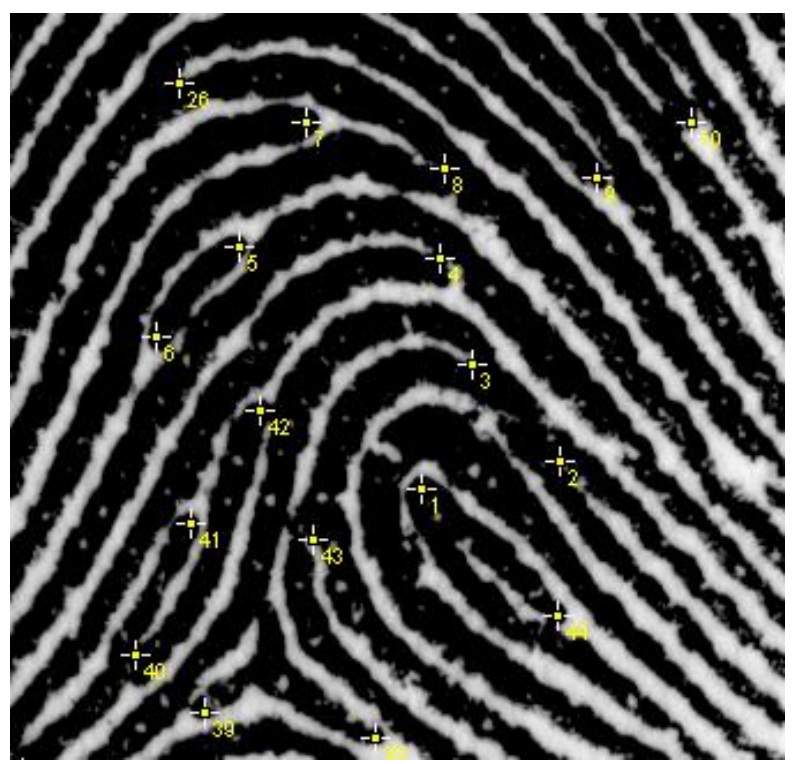

Figure 4. A section of an inked planar impression representing how minutiae were marked. Marker 1 represents the feature selected to be the origin of the fingerprint image.

The average $\mathrm{x}$ and $\mathrm{y}$ coordinates for each minutiae were then calculated. The average coordinates represent the 'true' position of the minutiae's expected location in the fingerprint. The locations of each minutiae were also represented in polar coordinates for versatility in data processing and visualization. For radial angles, zero degrees was set directly to the right of the origin feature and rotates counter-clockwise to 360 degrees. The compilation of true minutiae locations is used as the template for each finger. This template can then be used to determine the amount of variability that can be observed in a fingerprint.

\section{Distorted Images and Image Processing}

Images from the distortion videos were isolated on the camera itself [9]. The video was played back on the camera screen and paused at the peak of each distortional movement. The frame represented at the pause point was cut and saved from the video. This was performed for each movement and all repetitions of each movement. The image frames were then uploaded onto a computer and converted to TIFF files with Nikon ${ }^{\circledR}$ ViewNX2 ${ }^{\mathrm{TM}}$ software.

Each set of distortion images were opened in ImageJ, and the scale was set in the same manner as the inked planars. Each image was then split into its red, green, and blue color channels with the split channels function under the Image:Color tab. Contrast limited adaptive histogram equalization (CLAHE) was then performed on each color channel. A maximum slope of 3.00 and box size of 31 were found to be the best CLAHE settings for optimum image and contrast enhancement. CLAHE sets a limit to the number of pixels allowed for each intensity value. Pixels above this limit are redistributed equally to all intensities present in the image. The image improvements after CLAHE processing are shown in Figure 5. The distorted fingerprint images were flipped horizontally in relation to the inked planar impressions because they are videotaped from below. A horizontal flip of the distorted images was performed in ImageJ to orientate them as they would appear as latent prints, and to understand how distortions would affect latent or inked prints. 
The minutiae present in the 5 clearest distorted images were marked with the multi-point selector tool in the same manner as the inked planars. Once again, the $\mathrm{x}$ and $\mathrm{y}$ coordinates were extracted with the measure tool and loaded into an excel spread sheet. The minutiae coordinates were zeroed by subtracting the origin feature coordinates from those of each minutiae.

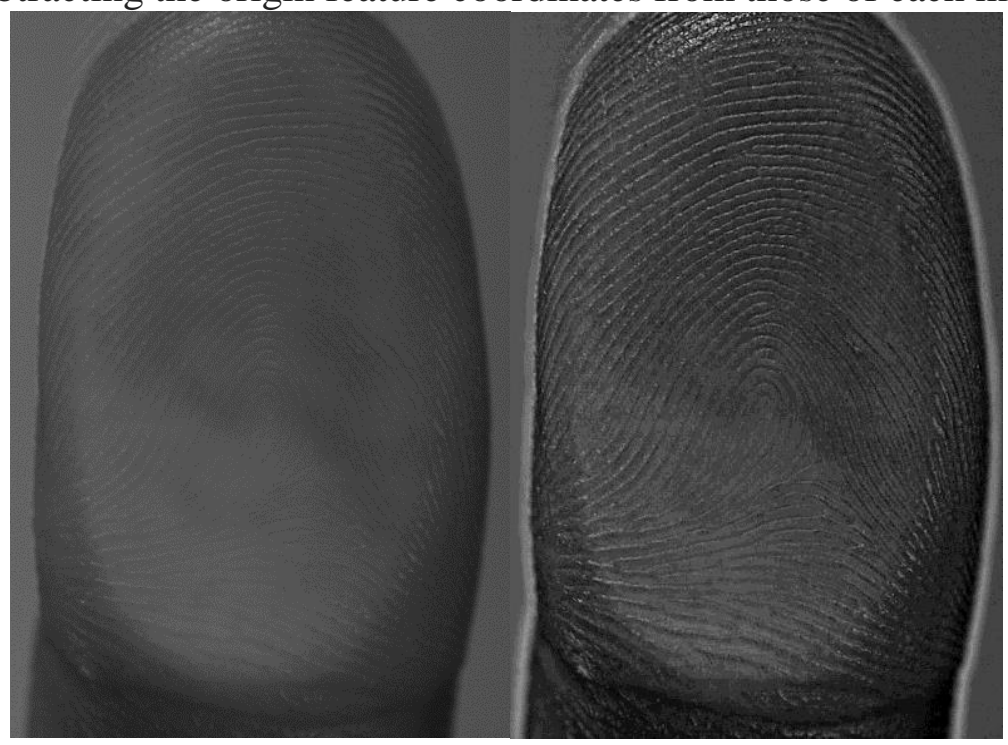

Figure 5. The fingerprint image on the left is the image isolated from the distortion video with no enhancement, and the image on the right is the image after enhancement with CLAHE.

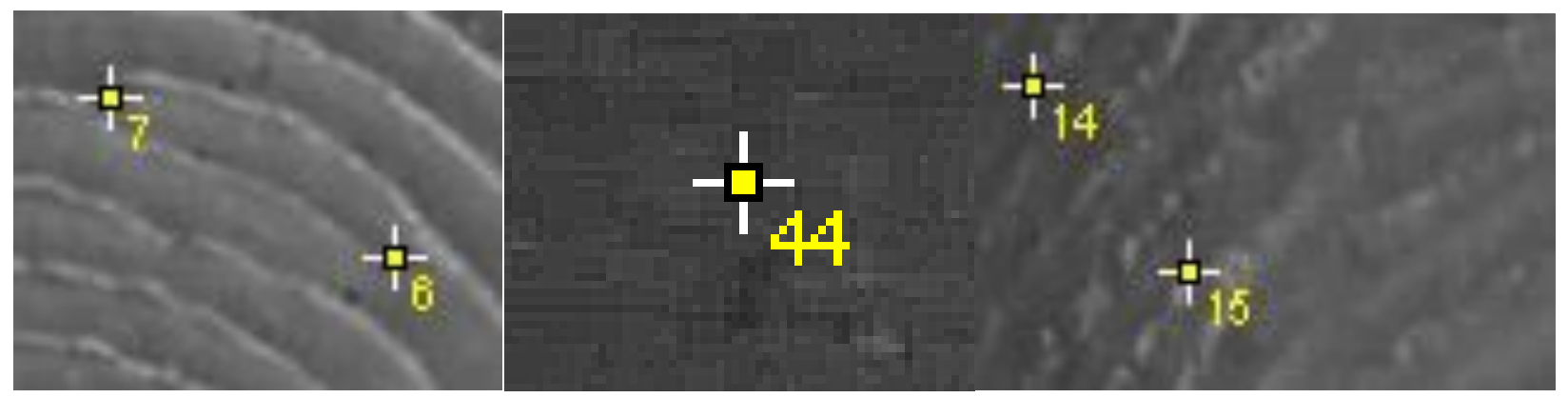

Figure 6. The image on the left shows a bifurcation and ending ridge of "Good" quality. The image on the right contains two minutiae falling outside the contact region; the edge of the contact region can be seen just to the right of minutiae 15. The image in the middle contains a minutiae of "Blurry" quality that cannot be accurately marked.

Due to the heavy distortion conditions and movements, some of the minutiae became too blurry to mark accurately or fell outside of the contacting finger region. The quality of minutiae was indicated in the Excel spreadsheet. An example of each minutiae quality can be seen in Figure 6. Only minutiae of quality "Good" were analyzed for this experiment as accurate locations cannot be extracted from "Blurry" minutiae, and minutiae falling "Outside Contact" would not be present in latent fingerprints or livescan recordings.

\section{Calculating Degree of Variability}

The minutiae coordinates from the minutiae template were subtracted from those of the distorted images to determine the $\mathrm{x}$ and $\mathrm{y}$ displacement resulting from the various distortional 
movements. The Euclidean distance between the distorted minutiae location and template location was calculated using the change in $\mathrm{x}$ and $\mathrm{y}$ coordinates. The Euclidean distance is calculated as follows:

$$
\mathrm{d}=\sqrt{\left(x_{d}-x_{t}\right)^{2}+\left(y_{d}-y_{t}\right)^{2}}
$$

where $\left(x_{d}-x_{t}\right)$ is the change in $\mathrm{x}$ location, $\left(y_{d}-y_{t}\right)$ is the change in $\mathrm{y}$ location, and $\mathrm{d}$ is the Euclidean distance.

The variability in minutiae orientation was investigated for a subset of the minutiae studied. Four minutiae were selected from each finger; minutiae exhibiting a displacement of 0.1 $\mathrm{mm}$ or less were targeted. These minutiae were selected to represent areas throughout the entire contacting finger region. The angle tool in ImageJ was used to determine the orientation of each minutiae. The orientation for bifurcations was traced along the path of the initial ridge, splitting the two new ridges. The orientation for ending ridges was traced along the path of the ridge in the direction of its termination. An example of each orientation can be seen in Figure 7.

The orientation for the minutiae was measured in each of the ten inked planar images and averaged to determine the 'true' orientation. The orientation was then measured in each of the distortion images and recorded. To determine the change in orientation of each minutiae, the true orientation was subtracted from that of the distorted minutiae.

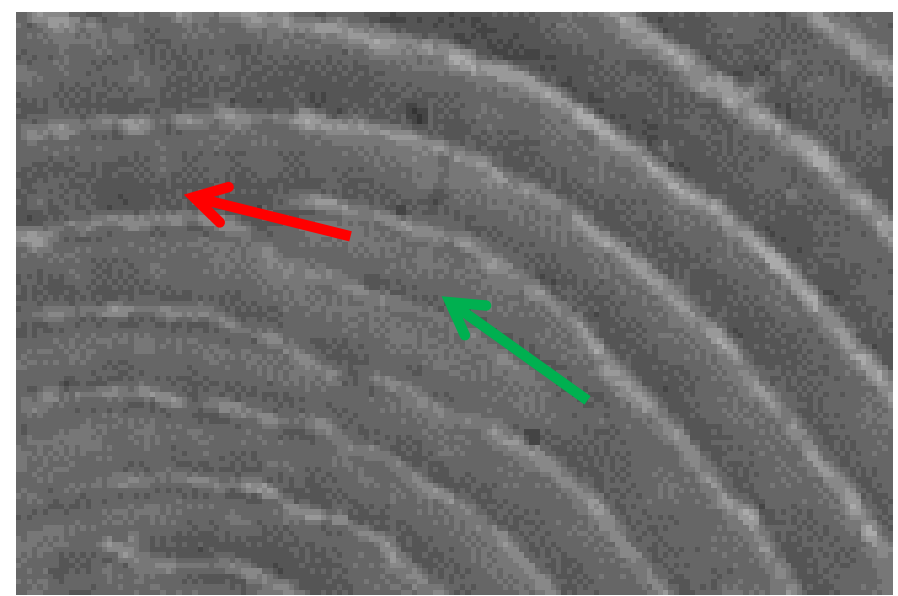

Figure 7. The red arrow depicts how the orientation was traced for ending ridges, and the green arrow represents how the orientation was traced for bifurcations.

\section{Distortion Map}

The finger was broken down into polar coordinate sections to assess the effect of distortion throughout fingerprints. The fingerprint area was divided into $1 \mathrm{~mm}$ radial bins and 60 degree angular bins. The radial angle bins were rotated 30 degrees from 0 to create the following radial angle sections: $330-30^{\circ}, 30-90^{\circ}, 90-150^{\circ}, 150-210^{\circ}, 210-270^{\circ}$, and $270-330^{\circ}$ (Figure 8 ). The radius of each region remained at $1 \mathrm{~mm}$ until an outer radial bound of $10 \mathrm{~mm}$ was reached. At this point all minutiae of radius greater than $10 \mathrm{~mm}$ were grouped into the same radial section. 
Minutiae of radius greater than $10 \mathrm{~mm}$ most often fall in the tip region, with few fingers having a contacting region of radius greater than $10 \mathrm{~mm}$ to the left and right. The lower bounds of the radial and angular bins are inclusive while the upper bound is excluded. For example, the radial bin from 2-3 mm contains all of the minutiae greater than or equal to $2 \mathrm{~mm}$ but less than $3 \mathrm{~mm}$.

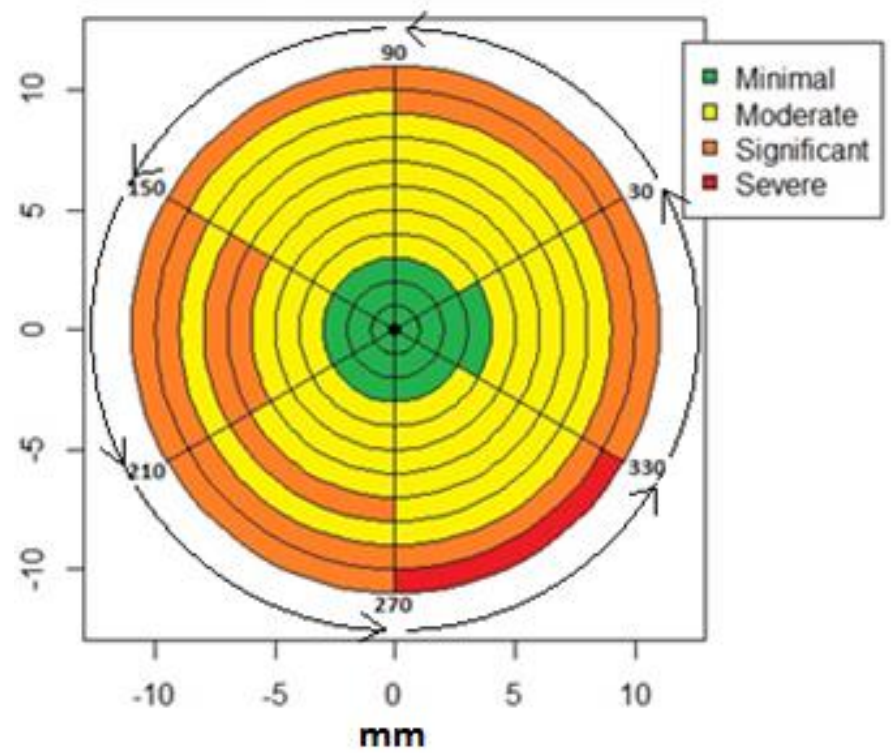

Figure 8. Example of polar sections used to create distortion maps seen in the results section. Angular sections rotate counter clockwise. The lower bounds of each radial and angular section are inclusive.

The radial and angular bins will be referenced by their lower bound for the purposes of the results and discussion. For example, the radial bin of 3 to $4 \mathrm{~mm}$ will be referred to as Radius 3 , and angular bin 330-30 degrees will be referred to as Angle 330. Each section of the distortion map was then color coded to represent the degree of variability observable across the fingerprint.

\section{Results}

\section{Justification of Section Determination}

Polar sections were initially set to $1 \mathrm{~mm}$ radial and $30^{\circ}$ radial angle sections. This amounted to 132 sections. An R script was then devised to separate the complete dataset into each $1 \mathrm{~mm}$ radial bin, and to calculate the mean Euclidean distance and standard deviation in each. The distribution of Euclidean distances of the displaced minutiae was then observed for each of the radial bins, and 50 of the 132 sections were randomly selected to test against the radial section data. The randomly selected sections means appeared to be separated by the set polar system.

Each of the $1 \mathrm{~mm}$ radial bins appeared to have different Euclidean means. Analysis of variance (ANOVA) was conducted in $\mathrm{R}$ to compare the Euclidean means at each radius interval (Appedix D). ANOVA is well suited for comparing more than two groups because it reduces the chances of false rejection that arises when conducting multiple t-tests [10]. ANOVA simultaneously compares multiple groups by comparing both the within group variance and 
between group variance through a least squares method [11]. First, each radius was tested for a normal distribution with a Q-Q plot (Appendix C). The data for each of the radii was determined to be sufficiently normal for proper ANOVA analysis.

The ANOVA test indicated a difference in means, but it does not specify between which radii. To determine which Radius means are different, a 95\% Confidence Tukey test was run to compare means. A Tukey test is a commonly associated post hoc test with ANOVA. It specifically determines which group means are significantly different through a series of pairwise comparisons of each group in the ANOVA. The difference of two means is divided by the standard error of the means to give a test statistic ( $p$-value) describing the similarity of the two means [10]. The adjusted p-values for each pairwise comparison in $\mathrm{R}$ indicate that all of the Radius Euclidean means are significantly different at the 95\% confidence level (Appendix D). Since the means for each radial bin were determined to be different, the radial intervals were set at $1 \mathrm{~mm}$.

The radial angle bins were then tested to decide if $30^{\circ}$ bins properly separated the data. The angular bins began at 0 and rotated counterclockwise to 360. There appeared to be a great deal of similarity in the mean Euclidean distances of neighboring angle sections throughout the finger. ANOVA analysis of the Euclidean means at each radial angle indicated a difference in means. The Tukey test results (Appendix D) indicate that not all of the angle bin means are significantly different from each other. Large adjusted p-values indicate two means are statistically the same at $95 \%$ confidence and the low values represent means that are different. The adjusted p-values showed neighboring sections Angle 330 and 0 to have indistinguishable means. Angle 0 could also not be distinguished from Angle 180 and 120.

In an attempt to prevent neighboring sections from containing the same means, the sections were increased to $60^{\circ}$ starting at 0 . Once again ANOVA analysis indicated a difference in some section means. A Tukey test showed angle 0 and angle 300 means to be indistinguishable as well as some diagonal section means (Appendix D). The radial angle bins were adjusted one more time by rotating the $60^{\circ}$ bins clockwise $30^{\circ}$. Here the bins began with $330-30^{\circ}$ and rotated counter clockwise. After ANOVA and Tukey analysis, no neighboring sections shared indistinguishable means at the $95 \%$ confidence level (Appendix D). Diagonal sections were sometimes indistinguishable from each other. It was determined that this was the best radial angle section to separate the data because no neighboring sections were the same. These angular sections also captured the varying ridge flow orientations throughout the finger.

The Euclidean distance values are not expected to be normally distributed in the angle sections as there is expected to be a large concentration of values closer to zero (slight skew). However, ANOVA can still properly handle some non-normal data as long as it is not bimodal or contain an irregular distribution [10]. Density plots were created for each of the angle section and can be found in Appendix C. They were found to satisfy the requirements for ANOVA.

The angle sections were named for the angle of the lower bound for each section. Angle 330 represents the span from 330 to 30, Angle 30 represents the span from 30 to 90, and so on. The set dimensions for the polar sections are of $1 \mathrm{~mm}$ in radius and $60^{\circ}$ radial angle. This creates a total of 66 sections comprising the contacting area of the finger. 


\section{Examiner Variability}

The degree of variability arising from the manual marking of minutiae was examined. The origin and 10 minutiae from an inked planar impression were marked 10 separate times. After the minutiae were marked for the first time this image was saved and used to mark the minutiae for the next 9 markings. This simulates the way in which inked and distorted images were marked throughout the study. The minutiae locations varied by an average of $0.018 \mathrm{~mm}$ or approximately 0.75 pixels. A maximum variation of $0.046 \mathrm{~mm}$ (approx. 1.8 pixels) was observed for the set of marked minutiae. This degree of variability is negligible in comparison to the variability arising from the recording of multiple planar impressions and the effects of the various distortion conditions discussed below.

\section{Variability of Inked Planar Impressions}

The variability of minutiae locations in inked planar impressions was analyzed with the mean Euclidean distance between minutiae and their true template locations. A clear radial property was noted for the amount of variability observed. The variability in possible minutiae location increased as the radius of the minutiae increased. The mean Euclidean values for each of the radial bins are presented in Table 1. The mean Euclidean distance is always greater than that of the preceding radii. The global Euclidean mean was $0.18 \mathrm{~mm}$ with a standard deviation of $0.17 \mathrm{~mm}$. The mean Euclidean distance of minutiae in each of the angular sections remains relatively constant, falling between 0.15 and $0.19 \mathrm{~mm}$. This was expected as there is limited directional distortion in the recording of inked planar impressions. Less than 5 percent of the total minutiae observed in any of the 10 repeated impressions exhibited displacement of more than $0.50 \mathrm{~mm}$.

\begin{tabular}{cccc}
\multicolumn{4}{c}{ Variability of Minutiae Location in Inked Planar } \\
Impressions \\
\hline Radius & $\begin{array}{c}\text { Mean Euclidean } \\
(\mathrm{mm})\end{array}$ & s.d. & Observations \\
\hline 0.001 & 0.043 & 0.029 & 380 \\
1 & 0.067 & 0.051 & 940 \\
2 & 0.090 & 0.063 & 1030 \\
3 & 0.12 & 0.10 & 1600 \\
4 & 0.15 & 0.11 & 1820 \\
5 & 0.18 & 0.15 & 2020 \\
6 & 0.21 & 0.19 & 1740 \\
7 & 0.23 & 0.19 & 1490 \\
8 & 0.25 & 0.19 & 1110 \\
9 & 0.28 & 0.23 & 810 \\
10 & 0.30 & 0.21 & 970 \\
\hline
\end{tabular}

Table 1. Mean Euclidean distance of minutiae in the radial bins of inked planar impressions. Mean values are reported with their standard deviations. 
The variability was further broken down into its $\mathrm{x}$ and $\mathrm{y}$ components through the $\mathrm{x}$ and $\mathrm{y}$ standard deviations of the minutiae coordinates. Both values appear to increase at approximately the same rate until a radius of $7 \mathrm{~mm}$ is reached (Figure 9). At Radius 7 the y standard deviation appears to level off at approximately $0.17 \mathrm{~mm}$, while the $\mathrm{x}$ standard deviation continues to increase.

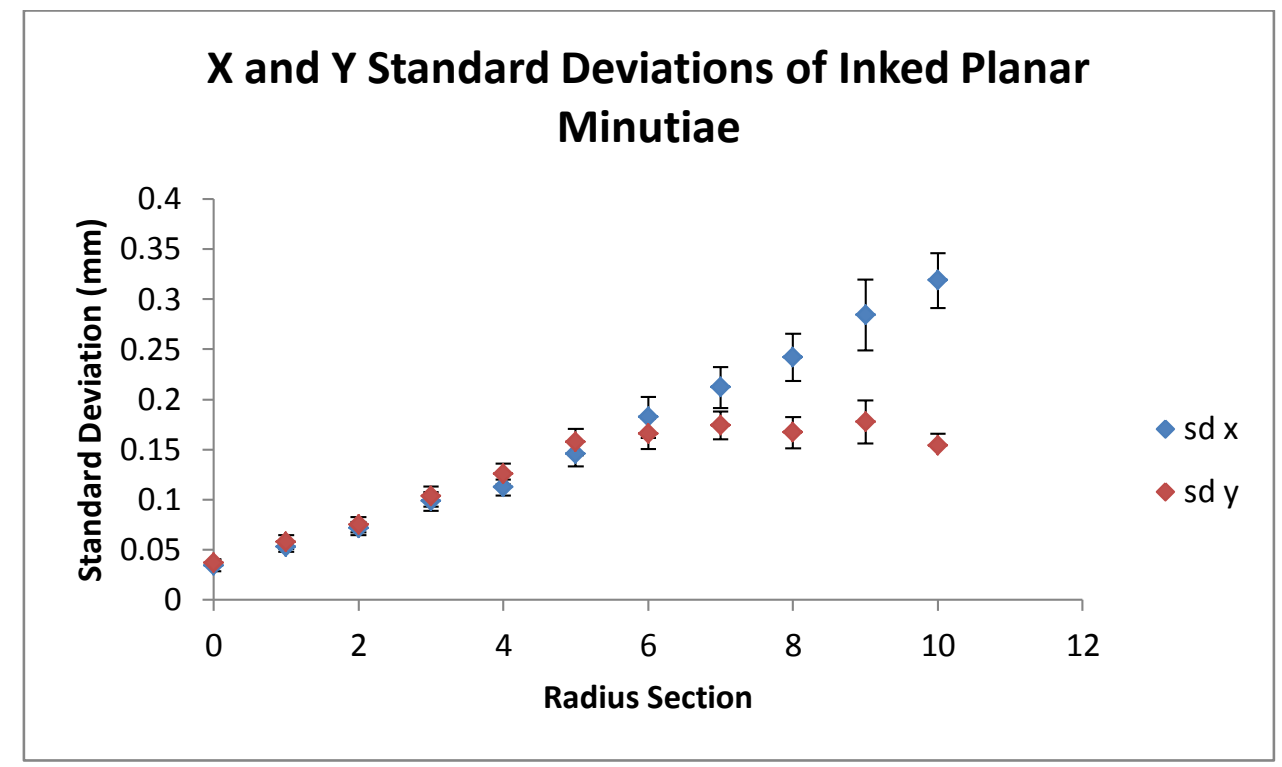

Figure 9. The $x$ and y standard deviations of minutiae in inked planar impressions in each radial bin. The values are represented with a $95 \%$ confidence interval.

The trends observed for the standard deviations suggest minutiae less than $7 \mathrm{~mm}$ from the origin would lie in a more circular confidence zone, while minutiae greater than $7 \mathrm{~mm}$ in radius would have an elliptical confidence zone. This trend is observed in Figure 10 below. The area encompassed by the variability bounds is smaller and more circular in shape near the origin, and much larger and more elliptical towards the edge of the finger.

The larger variability of minutiae locations further from the core increases the chances of confidence regions to overlap for minutiae in close proximity to each other. This dilemma is illustrated in Figure 11. A zoomed in view of the two confidence regions shows an overlapping area. An automated matching system could have difficulty in properly assigning a minutiae falling in this overlapping area. The true locations of each minutiae are not included in the overlapping region of the confidence regions. 
Finger 1: Right Slant Loop

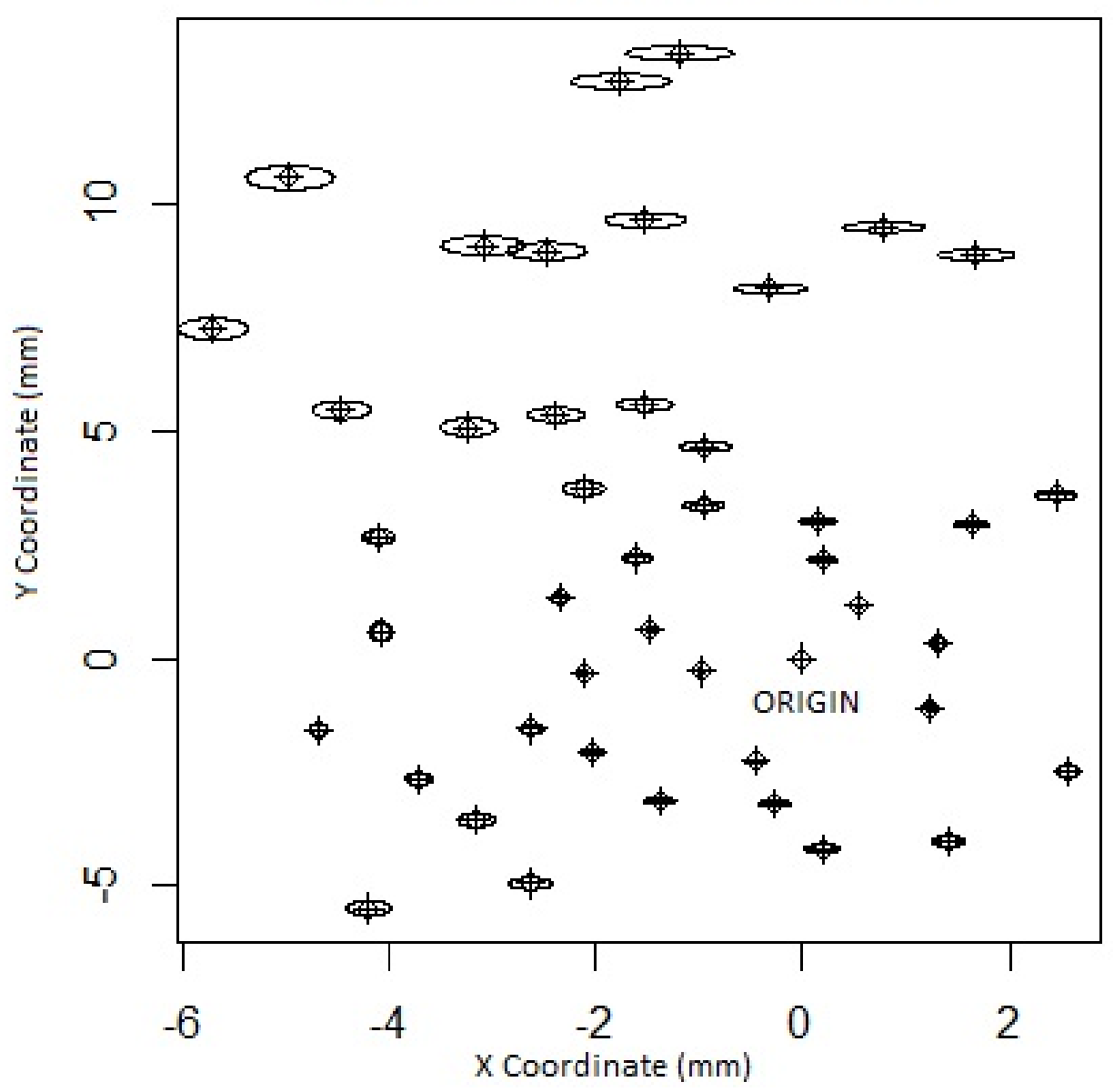

Figure 10. The cross hair points represent the true location of the minutiae in the template image for Finger 1 . The elliptical shapes represent the $95 \%$ confidence region of the minutiae true locations. The minor axis is twice the $y$ coordinate standard deviation and the major axis is twice the $\mathrm{x}$ coordinate standard deviation.

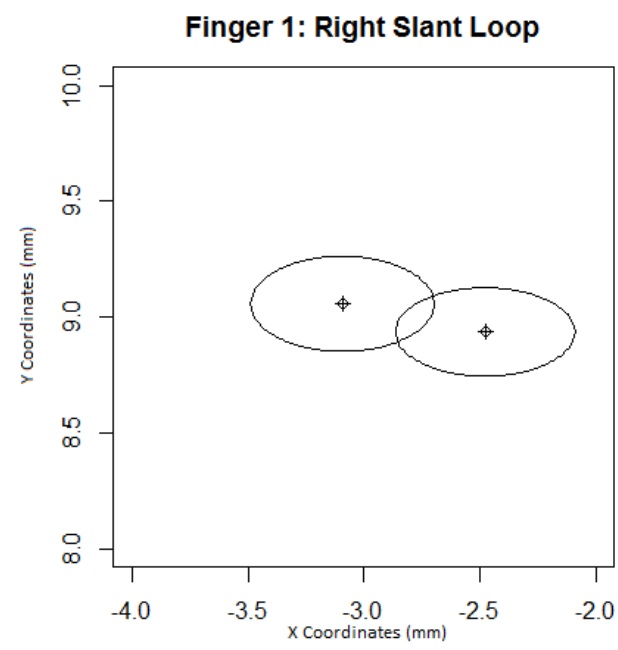

Figure 11. Overlapping variability ellipses of two inked planar minutiae. 


\section{Characterization and Directionality of Distortion Types}

It is important to note that all characteristics of each distortion are as they would appear in latent fingerprints. The distorted images were horizontally flipped to match the inked planars as this is how latents would be compared to inked impressions. Some distinct trends were noticed for the distortion conditions studied. The radial property observed in the inked planar impressions is even more noticeable with the various distortion conditions. The decrease in finger pressure permits greater variability in minutiae locations far from the origin. Minutiae close to the origin are displaced a smaller distance by the distortion movements, and a smaller overall variability in location is observed. As the minutiae move further from the origin, the variability in minutiae location increases and the distance displaced from their true locations increases as well. This trend can be observed in Figure 12.
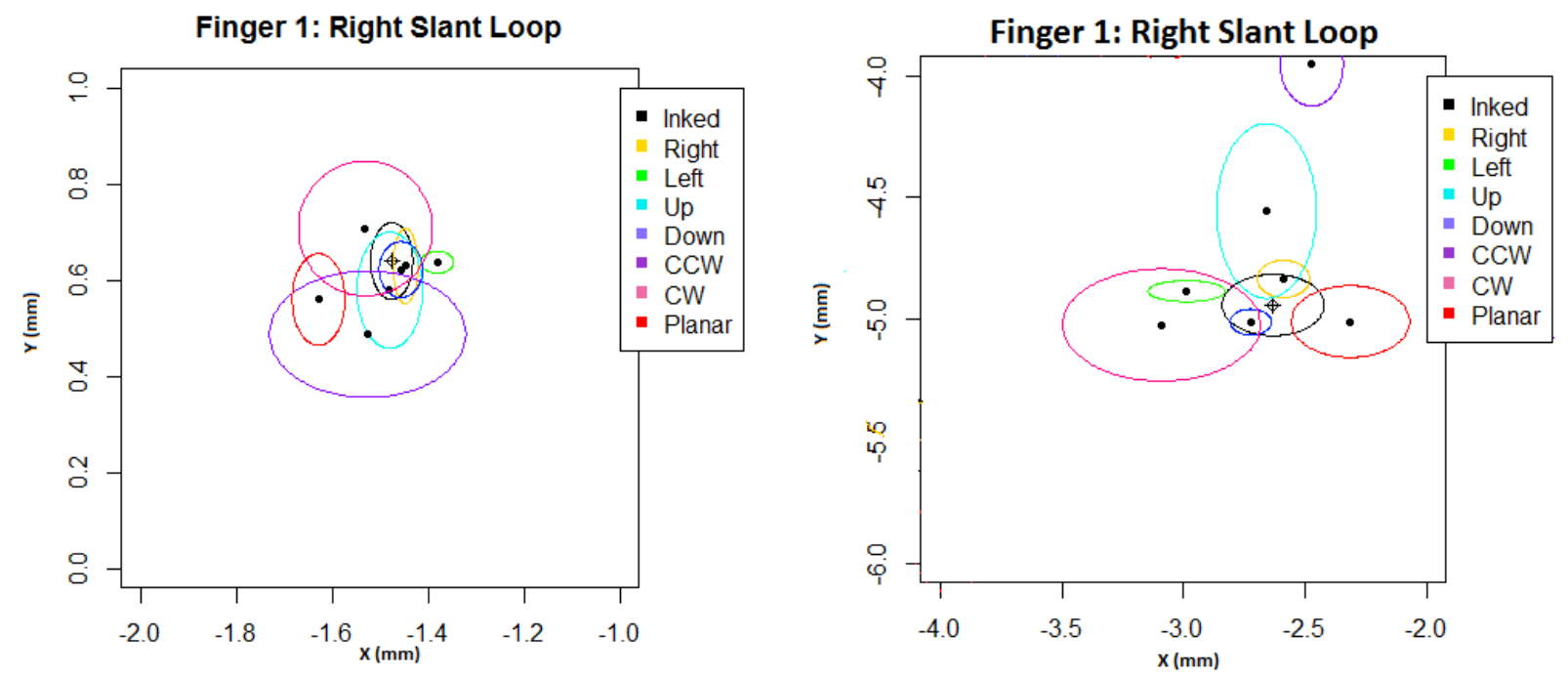

Figure 12. The image on the left contains a minutiae located approximately $1.5 \mathrm{~mm}$ from the origin and the image on the right contains a minutiae located nearly $6 \mathrm{~mm}$ from the origin of the same finger. The black points represent the mean location of the minutiae under each distortion condition with an elliptical variance bounds defined by twice the $\mathrm{x}$ and $\mathrm{y}$ standard deviations.

The distorted minutiae locations in the left image of Figure 12 fall close to or within the black variability boundary of the template minutiae location. A maximum variability of approximately $0.4 \mathrm{~mm}$ is observed with counter-clockwise torque (CCW). Minutiae locations in the right image often fall outside the black variability boundary of the true location, and exhibit larger variability in the distorted locations. A maximum variability bound of nearly $1 \mathrm{~mm}$ is observed with clockwise torque $(\mathrm{CW})$ and a maximum displacement of $1 \mathrm{~mm}$ with counterclockwise torque $(\mathrm{CCW})$. Heavy distortion can become a problem for minutiae located close in proximity as the variability increases.

Figure 13 depicts three minutiae in the bottom part of finger 30 . The various distortion ellipses show the large variability in minutiae locations for the three minutiae. The variability regions of each minutiae overlaps a significant portion of the area occupied by the minutiae above it. Minutiae have the potential to inhabit the area anywhere within or between their 
respective variability ellipses. This can make automated minutiae matching difficult for any system without an accurate registration. Of particular interest are the up translation variability ellipses. These ellipses have been isolated in the image on the right. One can see that the up translation ellipses are actually closer to the minutiae above their respective minutiae. A minutiae matching system would likely match minutiae 1 with 2 and minutiae 2 with 3 , and fail to match minutiae 3 without a proper registration of the two prints.
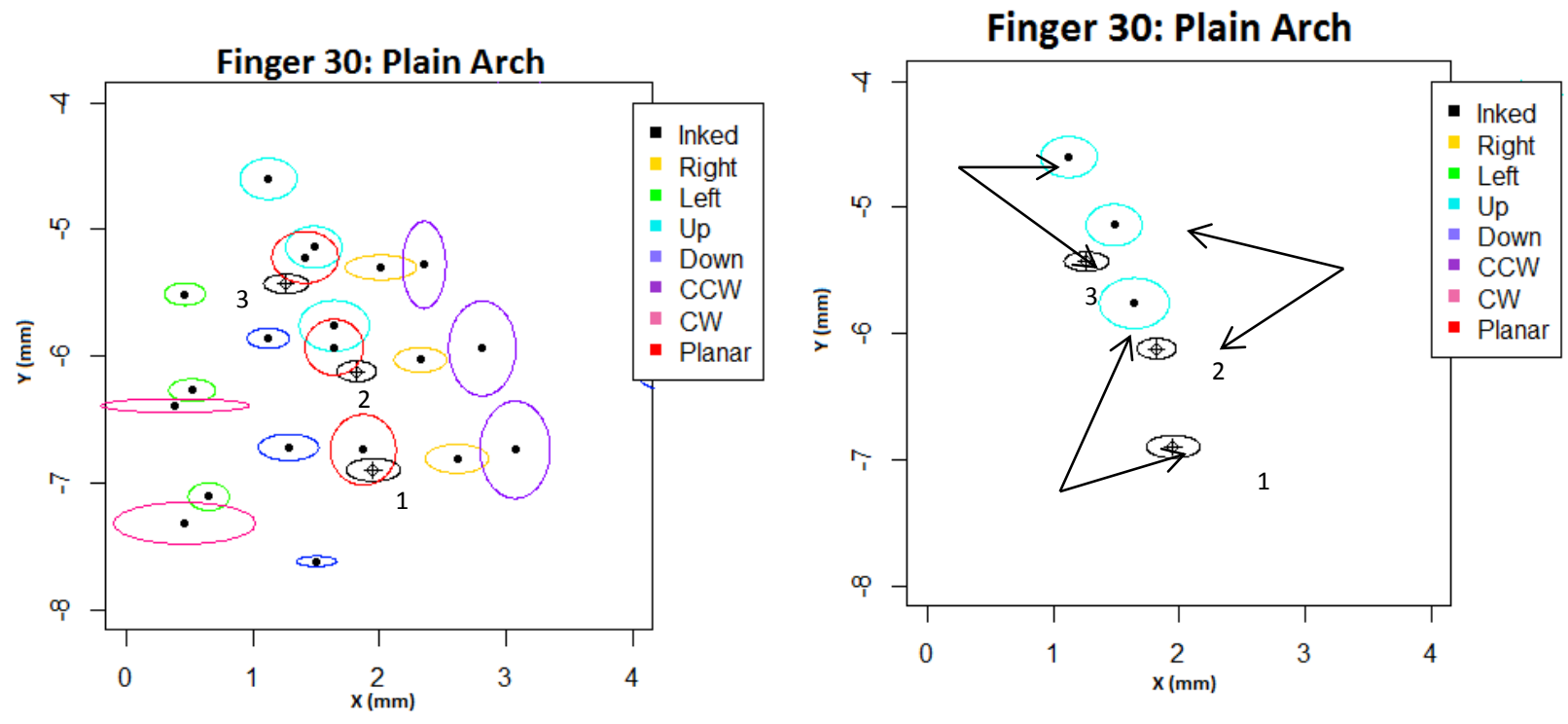

Figure 13. The image on the left contains three minutiae of finger 30 and the large range occupied by the minutiae after various distortions. The image on the right contains the same three minutiae with only up translation distortion. Arrows have been draw to show which up distorted ellipse is associated with which inked planar ellipse.

While minutiae of greater radius are displaced by larger distances, minutiae of shorter radius have the potential to exhibit much larger changes in their radial angle location. Figure 14 depicts how the potential change in radial angle of minutiae location decreases as radius increases. This trend suggests a variable size bounding box system as proposed by $\mathrm{He}[6]$ to be an effective system for minutiae matching. A bounding box of smaller radius and larger radial angle could be implemented for minutiae close to the core while a box of larger radius and smaller radial angle can be implemented for minutiae further from the core. These bounding boxes would capture the differences in variability observed throughout the fingerprint while encompassing the same area. 


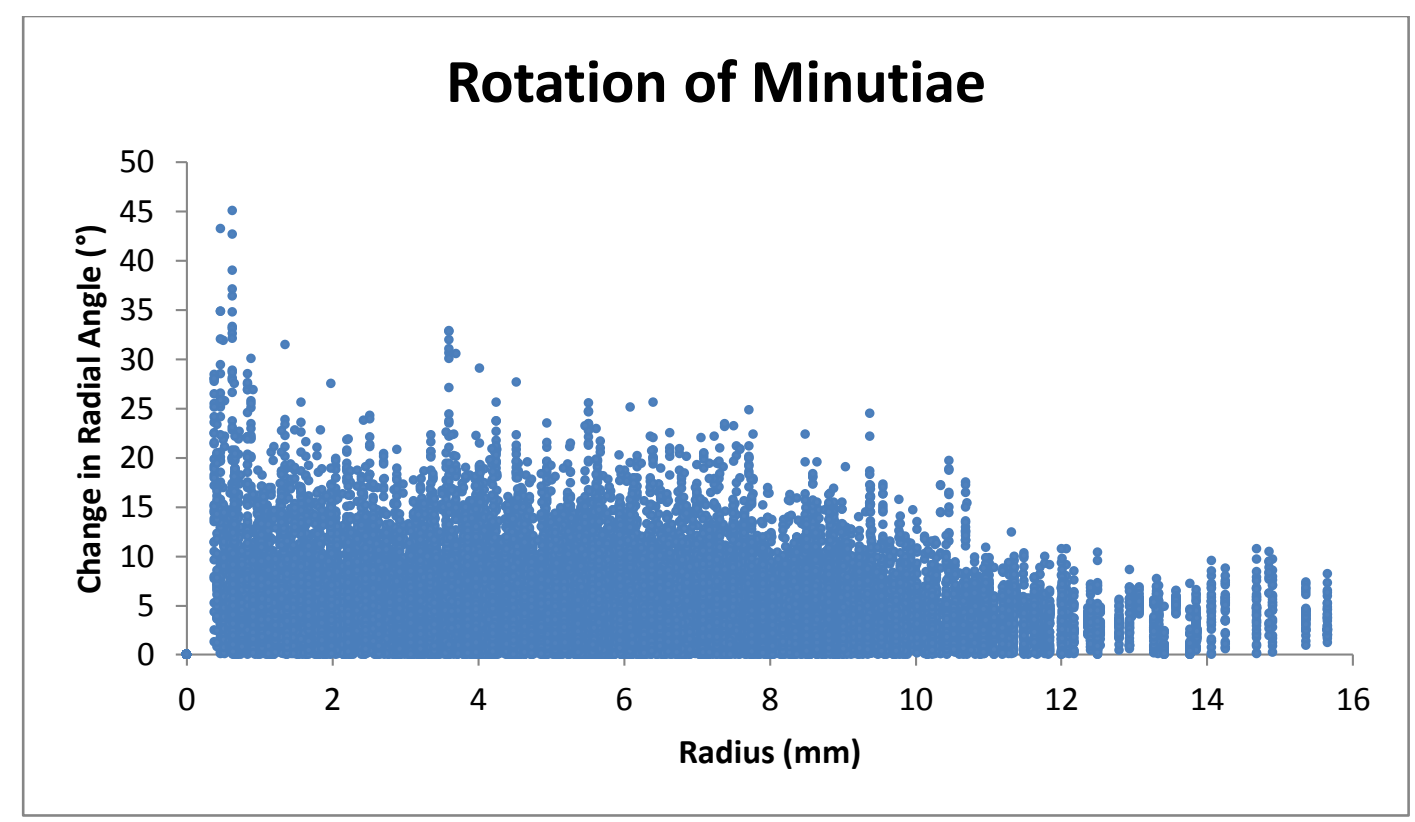

Figure 14. Minutiae of smaller radius have the potential to be rotated much more about the center of the finger than minutiae of large radii.

The minutiae locations under planar glass distortion (placement of finger on glass under heavy deposition pressure with no lateral or torque movements) are typically closest to the true minutiae locations. The planar glass distortion typically produces the least variability in minutiae locations, and the variability ellipse often overlaps with that of the true inked planar ellipse Left and right translation, up and down translation, and the torque distortions exhibit mirrored effects throughout the contacting finger region.

A left translation of the finger causes a general left displacement of minutiae in respect to the true location, and a right translation causes a general right displacement of minutiae. In the lower parts of the finger where the ridges remain flat, there is more of a directly horizontal displacement of the minutiae with a minor vertical component. In the regions above and to the left and right of the core, the minutiae displacement contains a more prevalent vertical component. This can be attributed to the arching ridges that enter on one side of the finger, rise towards the center, and descend and flow out the opposite side of the finger.

Minutiae in the upper arching area of these ridges experience an increased upward component in their position variability. Minutiae falling in the regions where the ridges flow down and out of either side of the finger experience a downward component in addition to the principle horizontal displacement. This effect is more pronounced in tall loop patterns as the arching of the ridges is steeper. The various regions are highlighted in Figure 15. 


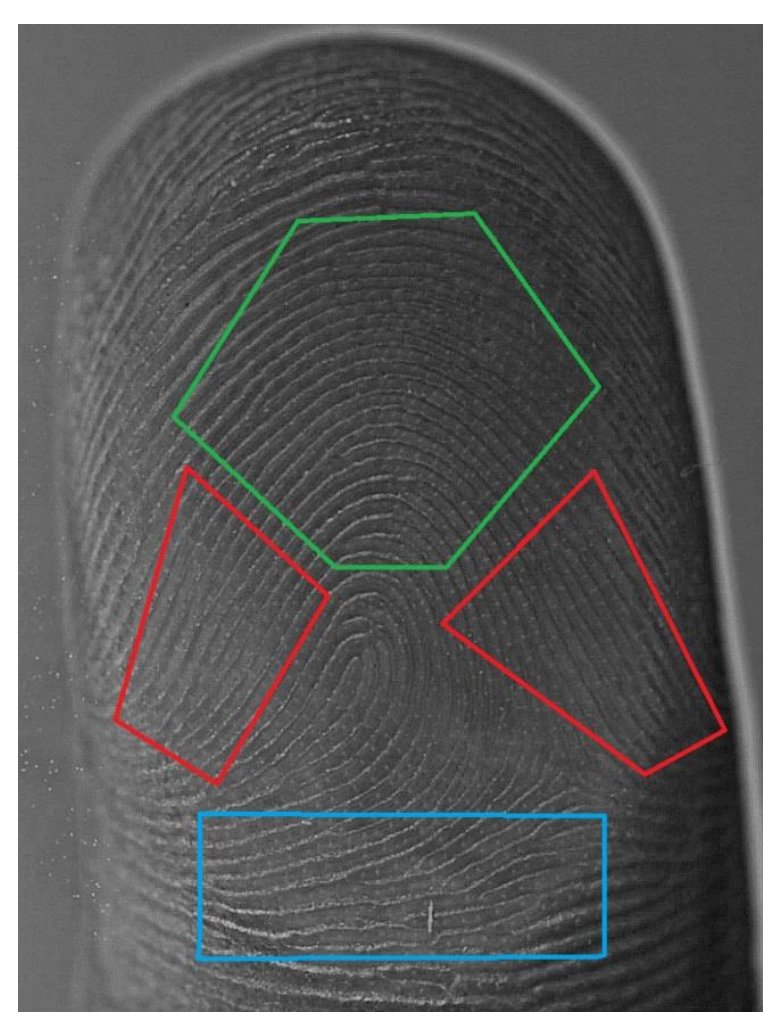

Figure 15. The blue region indicates the area where variability is mostly horizontal for left and right translations of the finger. The red regions indicate areas where an increased downward component arises and the green region indicates the area where an upward component arises.

Up and down translation of the finger produce an upward and downward displacement of minutiae respectively. In the parallel flowing ridges below the core, this displacement is mainly in the vertical direction with a small amount of horizontal displacement. The amount of horizontal component increases for minutiae in the arching ridges of the finger. Minutiae on the right half of the arching ridges have an increased rightward displacement in addition to the primary vertical component. Minutiae on the left half of the arching ridges experience an increased leftward displacement in addition to the primary vertical displacement (Figure 16).

Clockwise and counter-clockwise torque distortions produce distinct patterns in their displacement of minutiae. Counter-clockwise torque displaces minutiae up and to the right in the blue area of Figures 16. Moving counter-clockwise through the finger, minutiae are gradually displaced more vertically and begin to move to the left in the upper regions of the finger. As minutiae reach the upper left region (green area of Figure 16) they begin to exhibit a more prominently left and slightly downward displacement. Clockwise torque affects minutiae location oppositely to counter-clockwise torque. Minutiae in the lower regions of the finger are displaced to the left and down. Moving clockwise through the print the minutiae are displaced more vertically until reaching the upper portion of the finger. Minutiae in the upper right region (red area of Figure 16) begin to exhibit a more prominently right and slightly downward displacement. This can be best visualized by picturing minutiae as they lie on a circle and their displacement occurring tangent to their position on the circle. 


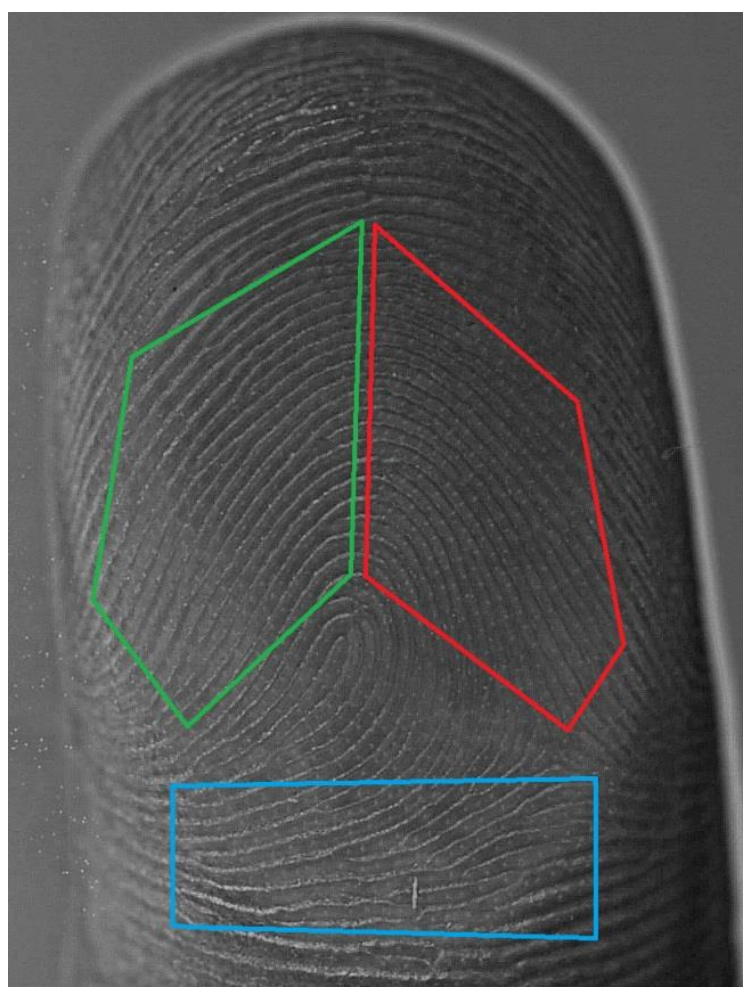

Figure 16. Minutiae in the blue region experience a primarily vertical displacement from up and down translations. An increased rightward component is experienced in the red region and an increased leftward component is experienced in the green region.

The effects of the various distortions behave consistently across the various pattern types with some slight variations in intensity. An important factor in the consideration of how minutiae are displaced is the overall ridge flow in the various regions. Loops and plain whorls tend to have more prominently arching/curving ridges, while plain arches tend to have more subtle arching ridges. Complete variance ellipse images for Fingers 1, 14, and 30 can be found in Appendix B.

\section{Distortion and Pattern Type}

The quantitative effects of all 7 distortional conditions were observed for the three pattern types. The mean Euclidean distance was calculated for each of the radial and angular bins as a means to quantify the range of variability in these sections. A total of 45,487 "Good" minutiae displacement observations were made for the 7 distortion conditions. Right slant loops, plain arches, and plain whorls all exhibited increasing Euclidean distance values as the radius of the minutiae increased. The variability of the three pattern types appear to increase at similar rates until a radius of $4 \mathrm{~mm}$ is reached; after $4 \mathrm{~mm}$, right slant loops exhibit lower mean values than both arches and whorls (Figure 17). Loop minutiae are displaced by up to $0.20 \mathrm{~mm}$ less than arches and up to $0.33 \mathrm{~mm}$ less than whorls at these intervals.

Two factor ANOVA (or analysis of covariance/ANCOVA) and Tukey test indicate that the right slant means are significantly different than those of plain arches and whorls for all radii greater than $4 \mathrm{~mm}$ with $95 \%$ confidence. The one exception is the mean value of right slant loops cannot be distinguished from that of plain whorls at $6 \mathrm{~mm}$. 


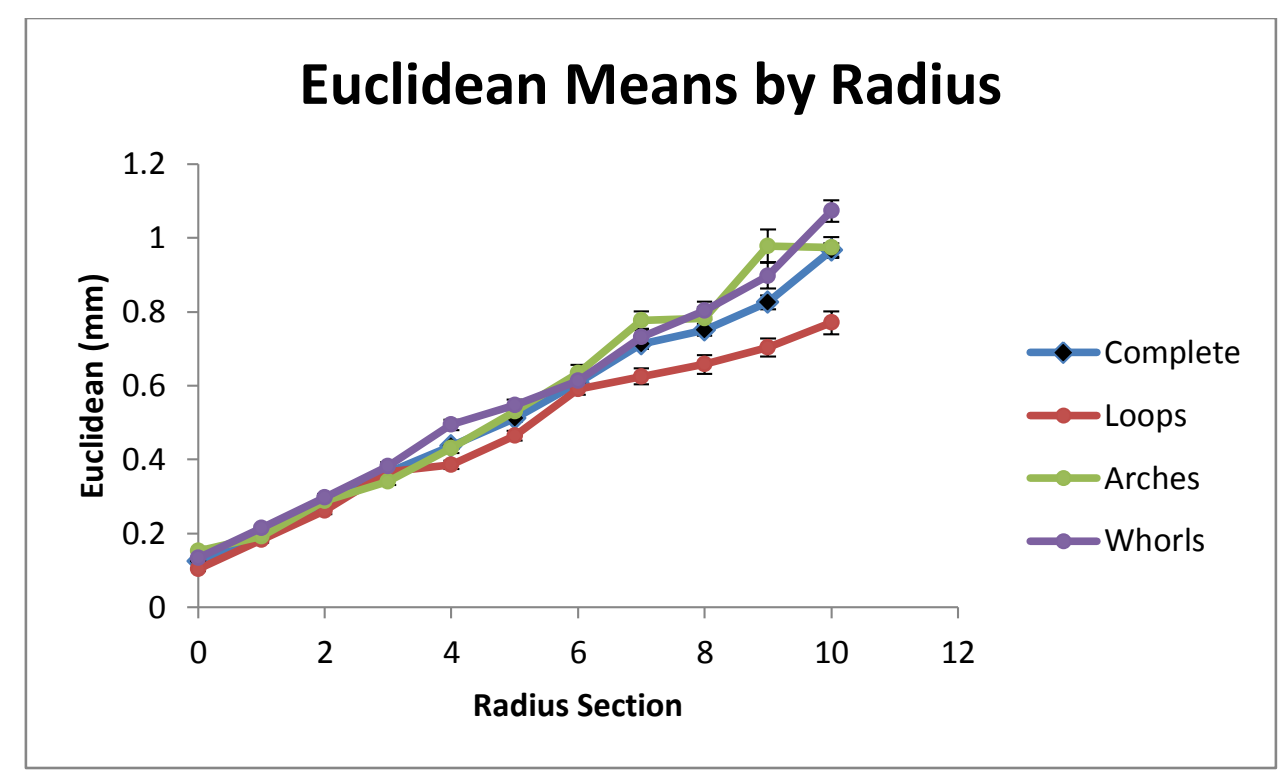

Figure 17. Mean Euclidean distances of minutiae displaced by the collective set of distortion conditions. Mean values are represented with a $95 \%$ confidence interval. The number of minutiae observations per Radius can be seen in Table 3.

The mean Euclidean values for each of the pattern types, as well as the entire set of fingers, are presented in Table 2. The global means for the complete dataset, right slant loops, plain arches, and plain whorls are $0.53 \mathrm{~mm}, 0.47 \mathrm{~mm}, 0.55 \mathrm{~mm}$, and $0.58 \mathrm{~mm}$ respectively. These global means give a general indication of the greater variability seen in plain arches and whorls. The maximum distortion values and number of observations by radius for each pattern are presented in Table 3. Most of the extreme distortion values are observed in the lower parallel flowing ridges of the fingers. This can be attributed to what appears to be a greater flexibility of the skin in the lower region when viewing the distortion videos.

\begin{tabular}{ccccccccc}
\multicolumn{8}{c}{ Mean Euclidean Distance of Distorted Minutiae by Radius } \\
\hline Radius & $\begin{array}{c}\text { Complete } \\
(\mathrm{mm})\end{array}$ & s.d. & $\begin{array}{c}\text { Loops } \\
(\mathrm{mm})\end{array}$ & s.d. & $\begin{array}{c}\text { Arches } \\
(\mathrm{mm})\end{array}$ & s.d & $\begin{array}{c}\text { Whorls } \\
(\mathrm{mm})\end{array}$ & s.d. \\
\hline 0.001 & 0.12 & 0.084 & 0.10 & 0.059 & 0.15 & 0.098 & 0.13 & 0.092 \\
1 & 0.20 & 0.12 & 0.18 & 0.12 & 0.19 & 0.11 & 0.21 & 0.13 \\
2 & 0.28 & 0.17 & 0.26 & 0.17 & 0.29 & 0.16 & 0.30 & 0.17 \\
3 & 0.36 & 0.25 & 0.37 & 0.27 & 0.34 & 0.20 & 0.38 & 0.26 \\
4 & 0.44 & 0.29 & 0.39 & 0.27 & 0.43 & 0.26 & 0.49 & 0.32 \\
5 & 0.51 & 0.35 & 0.46 & 0.34 & 0.53 & 0.29 & 0.55 & 0.38 \\
6 & 0.61 & 0.39 & 0.59 & 0.38 & 0.63 & 0.39 & 0.61 & 0.39 \\
7 & 0.71 & 0.46 & 0.63 & 0.40 & 0.78 & 0.46 & 0.73 & 0.48 \\
8 & 0.75 & 0.47 & 0.66 & 0.43 & 0.78 & 0.45 & 0.80 & 0.50 \\
9 & 0.83 & 0.50 & 0.70 & 0.43 & 0.98 & 0.50 & 0.90 & 0.54 \\
10 & 0.97 & 0.50 & 0.77 & 0.43 & 0.97 & 0.46 & 1.1 & 0.53 \\
\hline
\end{tabular}

Table 2. Mean Euclidean distance values of minutiae displaced by the collective set of distortion conditions. Mean values presented with their standard deviations. 
Maximum Euclidean Distance of Distorted Minutiae

\begin{tabular}{ccccccc}
\hline Radius & Loops $(\mathrm{mm})$ & Observations & Arches $(\mathrm{mm})$ & Observations & Whorls $(\mathrm{mm})$ & Observations \\
\hline 0.001 & 0.26 & 551 & 0.48 & 279 & 0.49 & 457 \\
1 & 0.71 & 1028 & 0.72 & 987 & 1.5 & 1220 \\
2 & 1.1 & 1429 & 0.89 & 819 & 1.1 & 1314 \\
3 & 2.1 & 1835 & 2.0 & 1634 & 1.7 & 2009 \\
4 & 1.7 & 2317 & 2.4 & 1544 & 2.0 & 2187 \\
5 & 2.4 & 2549 & 2.4 & 1561 & 2.59 & 2515 \\
6 & 2.8 & 2250 & 3.0 & 1131 & 2.5 & 2125 \\
7 & 2.7 & 1380 & 3.4 & 1279 & 3.0 & 1974 \\
8 & 3.0 & 1142 & 3.3 & 817 & 2.8 & 1555 \\
9 & 2.7 & 1170 & 3.9 & 496 & 3.3 & 911 \\
10 & 2.6 & 725 & 2.8 & 1085 & 3.6 & 1282 \\
\hline
\end{tabular}

Table 3. Maximum Euclidean distances for distorted minutiae observed under all distortion conditions for the three pattern types as well as the complete dataset..

A total of 15 minutiae recordings had a Euclidean distance of $3 \mathrm{~mm}$ or more. Of these 15 , thirteen were located in the lower parallel flowing ridges and along the very edge of the contacting region. The distortion videos show some of these minutiae to move slightly in and out of the contacting area throughout the various movements. This temporary freedom of movement, coupled with the increased flexibility of the lower ridge skin is likely responsible for the large displacement of minutiae location. Maximum displacements of approximately 2-2.5 mm are observed for minutiae located outside of the lower parallel flowing ridges and maintaining constant contact. Approximately 1 percent of the minutiae observed experienced a displacement of larger than $2 \mathrm{~mm}$ in any of the distortion replicates.

There are only minimal differences in the mean Euclidean values for minutiae in the lower finger region $\left(210-330^{\circ}\right)$ when compared to those in the upper finger region $\left(30-150^{\circ}\right)$. This can be seen in Table 4. While the means of the two regions are closely similar, the minutiae in the lower region exhibit noticeably larger extremes and standard deviations up to 50\% larger than the upper region. The difference in variability is more noticeable when observing each angular section in these radii. Angle 210 and 270 consistently exhibit larger Euclidean means than those of Angle 30 and 90. At Radius 6-10, the lower angle sections consistently exhibit mean values of $0.7 \mathrm{~mm}$ to more than $1 \mathrm{~mm}$, while the upper angle sections typically exhibit mean values from 0.3 to $1 \mathrm{~mm}$. These trends can be observed in the polar distortion maps in the Distortion Map section. 
Mean Euclidean Distance of Minutiae in the Lower and Upper Regions of Fingerprints

\begin{tabular}{ccccccc}
\hline Radius & Lower & s.d. & Observations & Upper & s.d. & Observations \\
\hline 0.001 & 0.12 & 0.075 & 472 & 0.14 & 0.098 & 450 \\
1 & 0.23 & 0.14 & 995 & 0.18 & 0.10 & 1342 \\
2 & 0.27 & 0.17 & 1321 & 0.27 & 0.15 & 1155 \\
3 & 0.37 & 0.26 & 2610 & 0.33 & 0.20 & 1464 \\
4 & 0.46 & 0.32 & 2643 & 0.39 & 0.25 & 1713 \\
5 & 0.51 & 0.36 & 3316 & 0.48 & 0.29 & 2057 \\
6 & 0.65 & 0.43 & 2583 & 0.54 & 0.32 & 2126 \\
7 & 0.73 & 0.50 & 2272 & 0.64 & 0.37 & 1710 \\
8 & 0.75 & 0.52 & 1973 & 0.74 & 0.39 & 1431 \\
9 & 0.86 & 0.58 & 1003 & 0.80 & 0.42 & 1484 \\
10 & 0.98 & 0.63 & 737 & 0.96 & 0.45 & 2355 \\
\hline
\end{tabular}

Table 4. Mean Euclidean value of minutiae found in the lower (210-330 ) region of the finger and upper (30$150^{\circ}$ ) region.

The seven distortion conditions appear to behave similarly for each pattern type when observed by radial bins. For right slant loops and plain whorls there appears to be increased variability with up translation, counter-clockwise torque, and clockwise torque movements. These distortions appear to create noticeably larger displacement of minutiae starting at Radius 3 with the difference increasing as the radius increases (Figures 18-19). These three distortions create the largest displacement of minutiae in plain arches as well, with clockwise torque imparting the most significant displacement of the three (Figure 20).

As expected the planar glass impressions had the smallest effect on minutiae locations. While an up translation of the finger produced some of the largest displacement in each pattern, a down translation of finger consistently produced displacement along the magnitude of planar glass impressions. Left and right translation of the finger caused moderate displacement, falling more towards the middle of the distortion set. The torque movements and up translation create upwards of $0.5 \mathrm{~mm}$ more variability than down or planar glass impressions at the different radii, and between $0.2-0.40 \mathrm{~mm}$ more variability than left and right translations.

Complete lists of the mean and maximum Euclidean values for each distortion can be found in Appendix A. Right slant loops routinely exhibit the smallest Euclidean means at almost every radius for each distortion with maximum values being split between plain arches and plain whorls. 


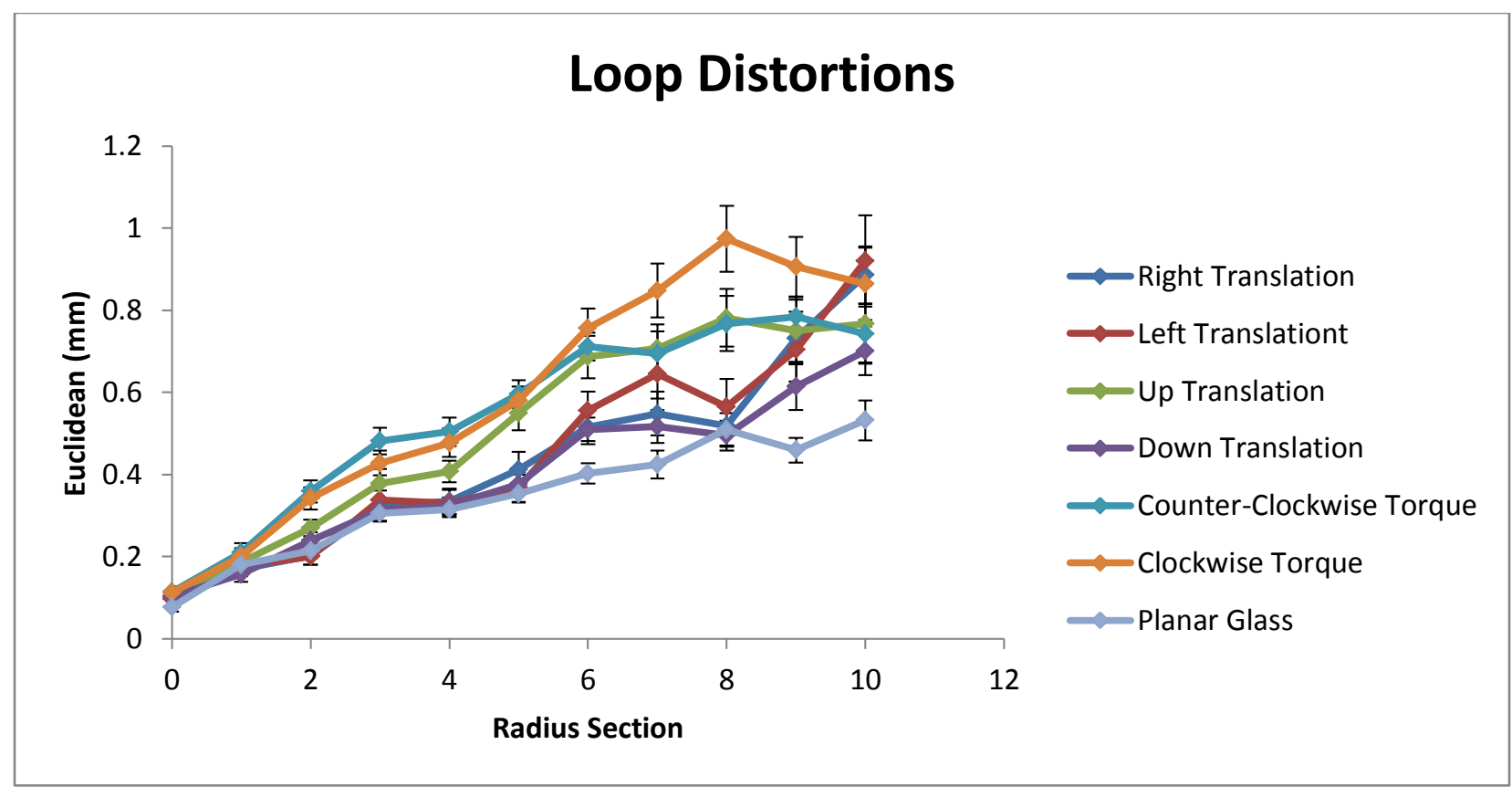

Figure 18. Mean Euclidean displacement of right slant loop minutiae for each distortion type. Mean values are presented with 95\% confidence. The fewest observations for right slant loops occurred in Radius 001, ranging between 75 and 80 minutiae observations for each distortion. Maximum observations ranged between 319 and 381 minutiae observations in Radius 4 and 5 for each distortion. The full list of observations by radial section can be found in Appendix A.

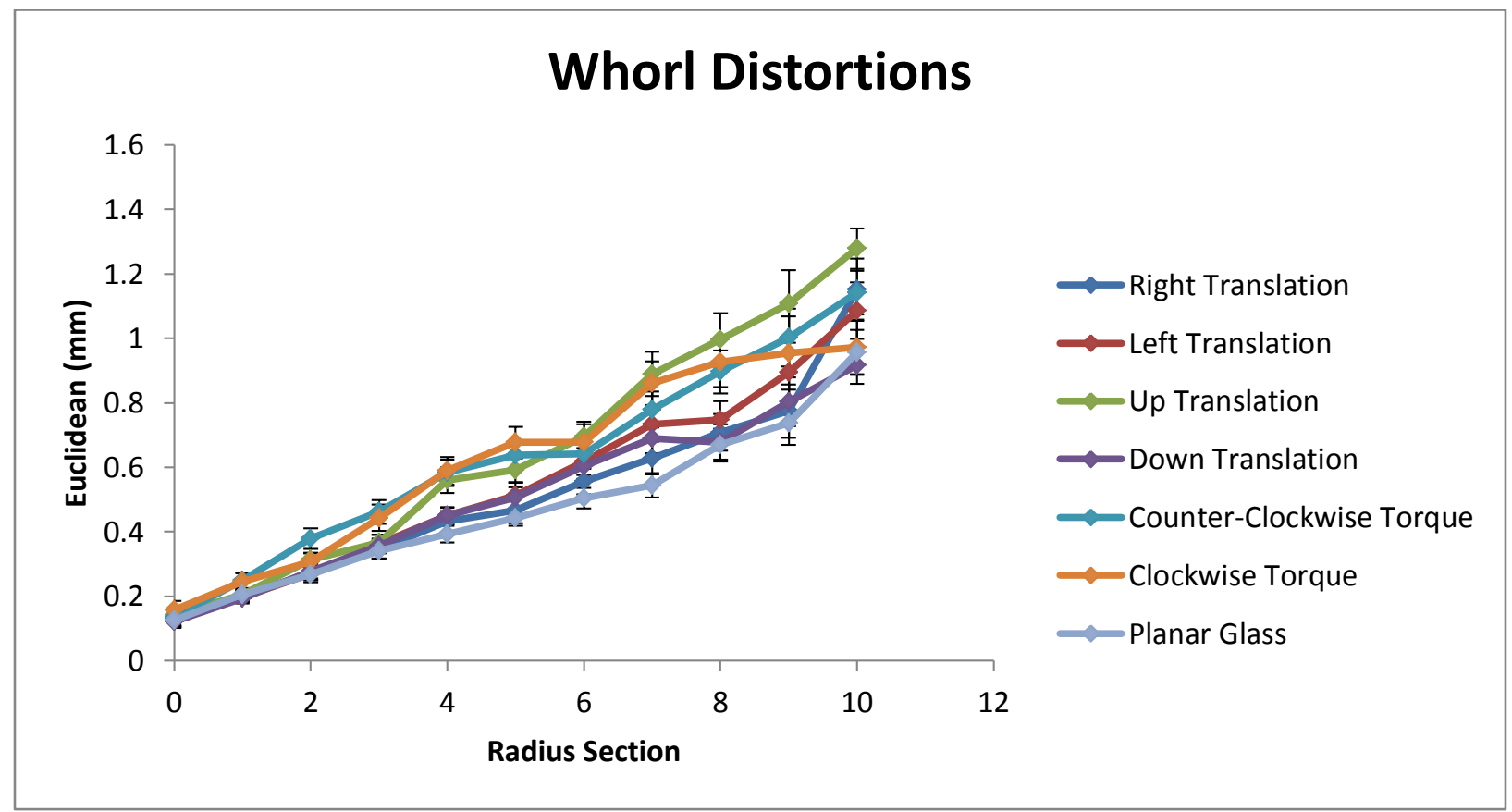

Figure 19. Mean Euclidean displacement of plain whorl minutiae for each distortion type. Mean values are presented with 95\% confidence. The fewest observations for plain whorls occurred in Radius 001, ranging between 39 and 40 minutiae observations for each distortion. Maximum observations ranged between 221 and 238 minutiae observations in Radius 3 for each distortion. The full list of observations by radial section can be found in Appendix A. 


\section{Arch Distortions}

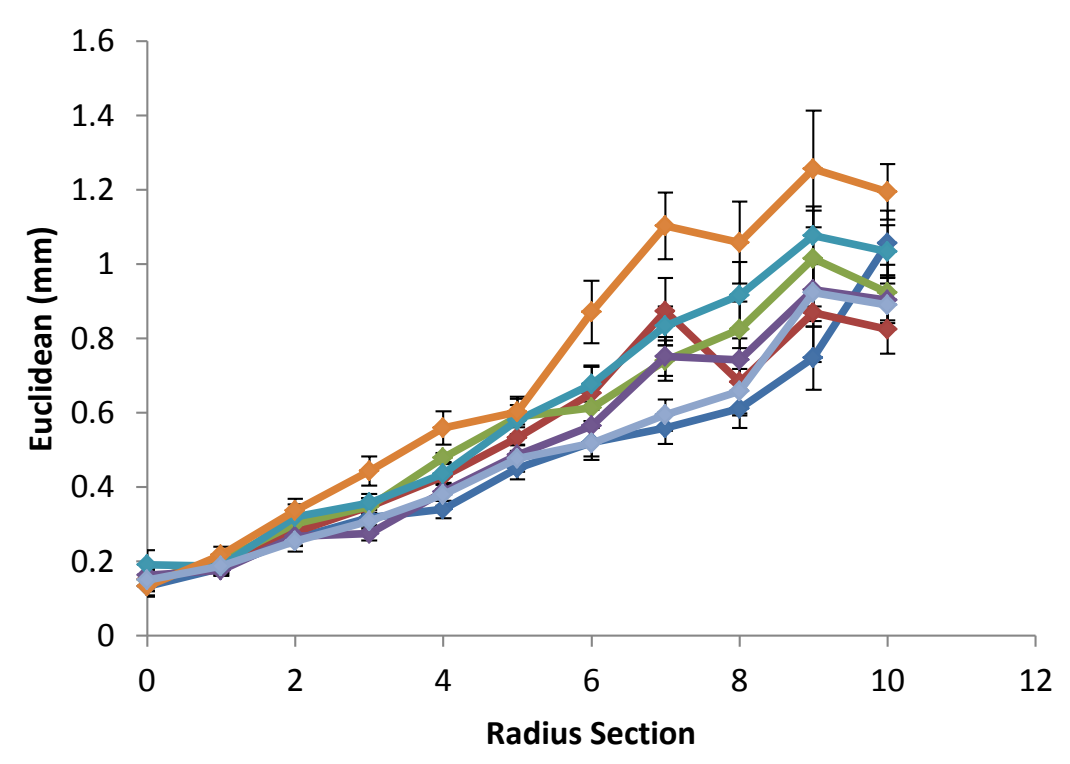

$\smile$ Right Translation

$\leftrightarrow$ Left Translation

$\leadsto$ Up Translation

$\leadsto$ Down Translation

$\leadsto$ Counter-Clockwise Torque

$\leadsto$ Clockwise Torque

$\leadsto$ Planar Glass

Figure 20. Mean Euclidean displacement of plain arch minutiae for each distortion type. Mean values are presented with 95\% confidence. The fewest observations for plain arches occurred in Radius 001, ranging between 62 and 67 minutiae observations for each distortion. Maximum observations ranged between 330 and 373 minutiae observations in Radius 5 for each distortion. The full list of observations by radial section can be found in Appendix

A.

While plain whorls typically exhibit larger displacement from each distortion type, the difference between the effects of each distortion is smaller at the different radii. This is likely due to the circular nature of ridges in plain whorls. This provides a relatively uniform ridge flow regardless of the directionality of the distortion applied. The ridge flow in plain arches and right slant loops creates different ridge orientations throughout the fingerprint. Right and left translations act directly on the ridges flowing down and out of the finger, and up and down translations act more directly on the arching ridges and lower parallel ridges of fingerprints.

The effects of each distortion on the different pattern types become much more obvious when observed by angular sections. The overall ridge flow in the various sections produce distinct trends for the different pattern types. For all three pattern types an overall decrease in variability occurs in Angle 150. Plain whorls also exhibit an overall decrease in Angle 330. These angular sections are typically occupied by the deltas of right slant loops and plain whorls. The variety of ridge orientations and ridge densities in these regions are likely responsible for these decreases in variability.

For plain arches, the amount of displacement is significantly lower in Angle 150 for all 7 distortions than any other section (Figure 21). It is not clear why variability is so much smaller in plain arches as they do not contain a delta formation. Perhaps there is an underlying reason based on the structure of the distal portion of the finger itself or there could be a kind of pseudo delta formation from the termination of ridges in this area, rather than just the orientation of ridges. The fewest number of minutiae observations occur in Angle 150 (51-92 for each distortion), but 
there should be a large enough sample size to give a realistic estimate of variability in this regions. A mean displacement of 0.27 to 0.40 is observed Angle 150 for all 7 distortions.

Clockwise and counter-clockwise torque create the most displacement in each section with up translation often second or third most. Clockwise torque produces the most variability in particular, displacing minutiae by nearly $0.4 \mathrm{~mm}$ more at some radii. Planar glass and down translation rarely produce more than $0.5 \mathrm{~mm}$ of displacement, and left and right translations produce a moderate amount of displacement (0.3 to $0.6 \mathrm{~mm})$.

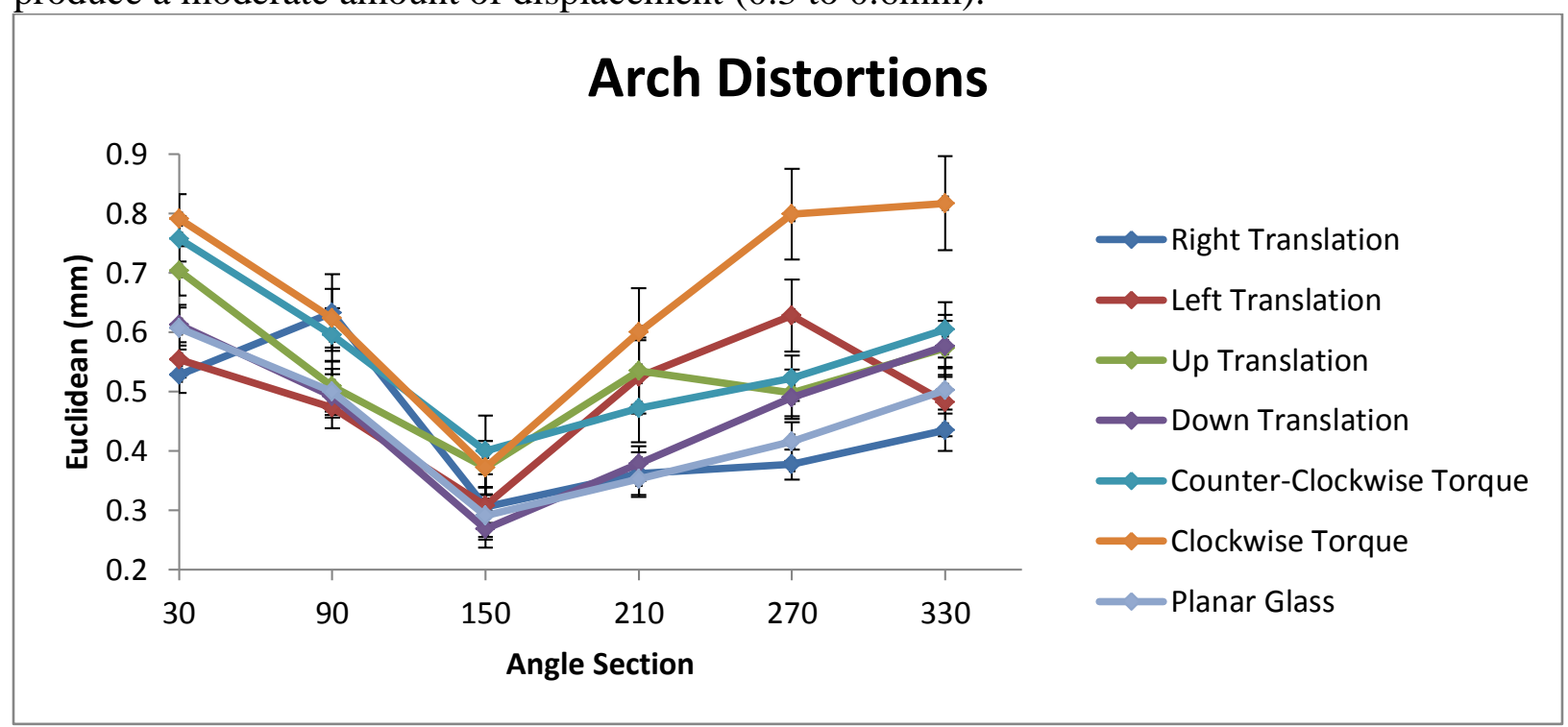

Figure 21. Mean Euclidean distance of plain arch displaced minutiae by angle section for each distortion conditions. Values presented with $95 \%$ confidence intervals. The fewest observations for plain arches occurred in Angle 150, ranging between 51 and 92 minutiae observations for each distortion. Maximum observations ranged between 435 and 480 minutiae observations in Angle 30 for each distortion. The full list of observations by angular section can be found in Appendix A.

Right slant loops display noticeably different trends in the areas affected by each distortion. Clockwise torque, counter-clockwise torque, and up translations produce $0.20-0.30$ $\mathrm{mm}$ more distortion in the lower portion of the fingerprint $\left(150-330^{\circ}\right)$ than they do in the upper $\left(30-150^{\circ}\right)$ regions. Left and right translation causes noticeably larger displacement of minutiae in the upper regions of the print than they do in the lower region (Figure 22). Differences of nearly $0.20 \mathrm{~mm}$ are observed. The displacement is also larger than that of clockwise torque, counter clockwise torque, and up translation in the upper regions. Down translation causes a relatively similar amount of displacement throughout the entire finger of magnitude similar to planar glass, and never reaching more than $0.45 \mathrm{~mm}$. There is also a relatively lower amount of displacement observed in Angle 150 (with the exception of up translation), an area typically inhabited by the delta of right slant loops. 


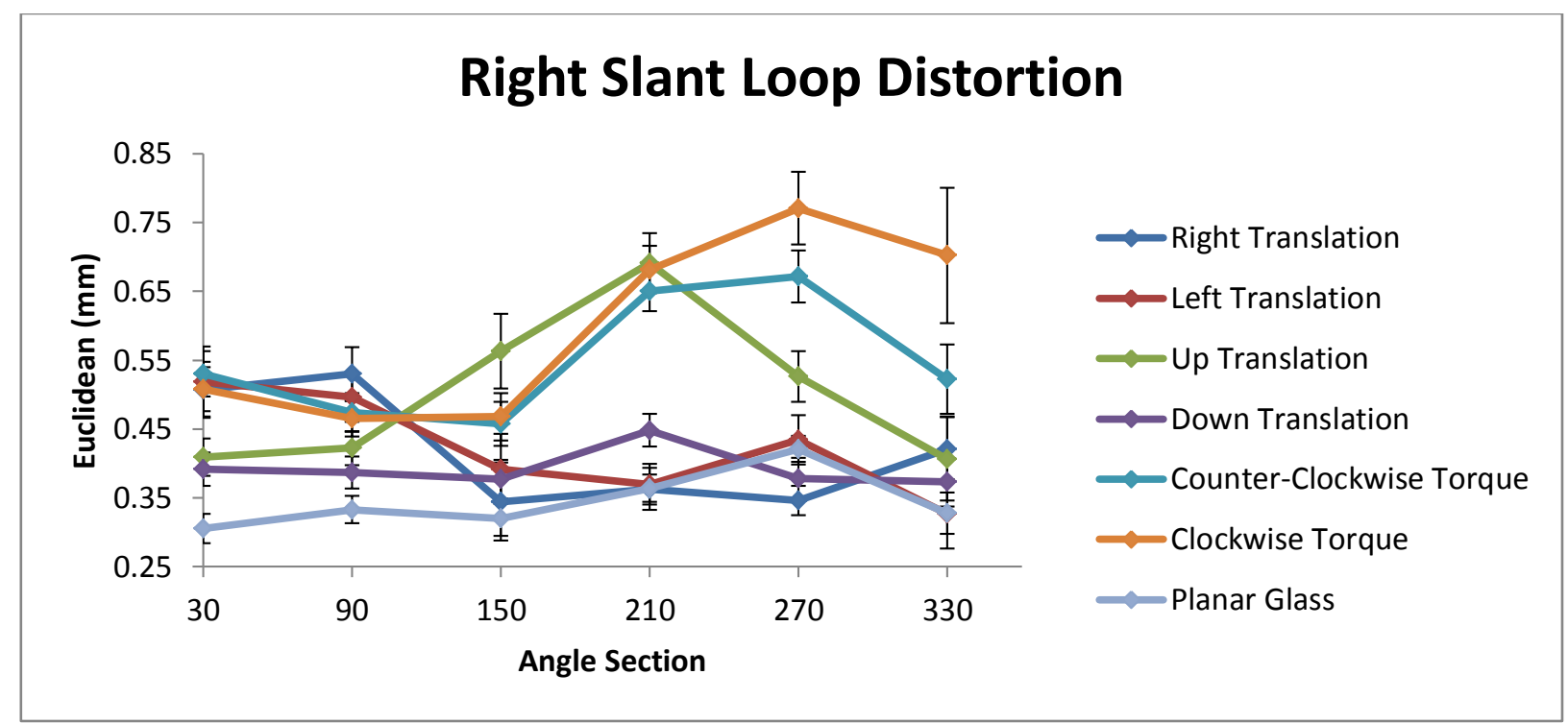

Figure 22. Mean Euclidean distance of plain arch displaced minutiae by angle section for each distortion condition. Values presented with 95\% confidence intervals. The fewest observations for right slant loops occurred in Angle 330, ranging between 96 and 161 minutiae observations for each distortion. Maximum observations ranged between 490 and 535 minutiae observations in Angle 90 for each distortion. The full list of observations by angular section can be found in Appendix A.

Less obvious trends are observed for the different distortion conditions in plain whorl areas. Left and right translation still exhibit larger displacement in the upper regions and up translation and torque movements still exhibit larger displacement in the lower regions.

However, there is a more uniform amount of variability observed across the entire print for some distortions (Figure 23). The smallest displacement caused by each distortion occurs in Angle 330; all distortion having means less than $0.50 \mathrm{~mm}$. Up translation and clockwise torque also show noticeably larger displacements at Angle 150 than the other distortions.

Table 5 presents the largest displacement of minutiae observed in each angle section for the three pattern types. The associated distortion is often in agreement with the overall trends observed, and is included in parentheses for each observation. The maximum Euclidean distance observed under each distortion for the three pattern types are provided in Table 6 . The largest displacement for each pattern type always results from one of the torque movements. Full values for each section by distortion are provided in Appendix A. 


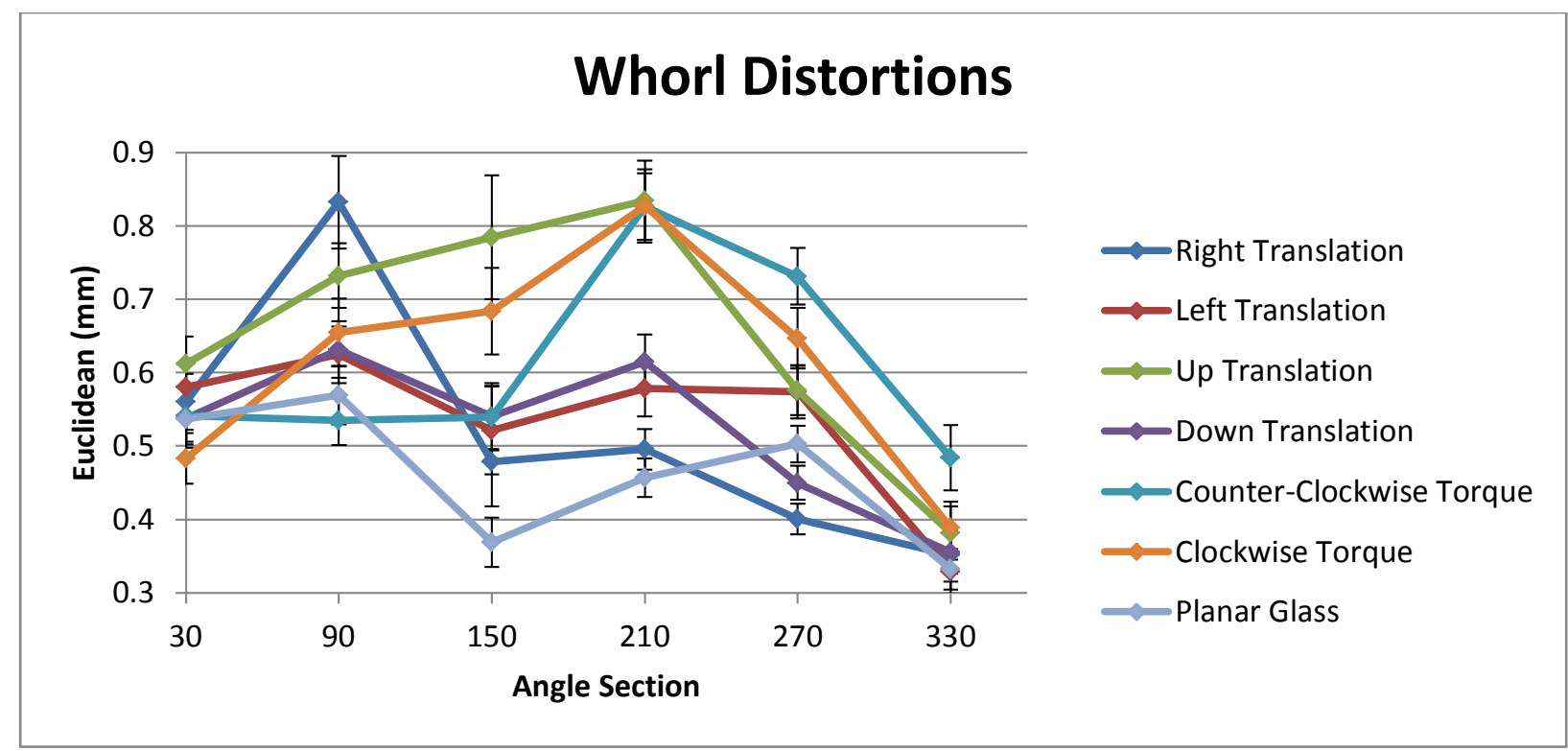

Figure 23. Mean Euclidean distance of plain whorl displaced minutiae by angle section for each distortion condition. Values presented with $95 \%$ confidence intervals. The fewest observations for plain whorls occurred in Angle 150, ranging between 169 and 226 minutiae observations for each distortion. Maximum observations ranged between 645 and 704 minutiae observations in Angle 270 for each distortion. The full list of observations by angular section can be found in Appendix A.

The trends observed suggest that distortion movements acting relatively perpendicular to the ridge flow of the different areas produces the largest displacement of minutiae. This is readily observed in the distortion maps presented in the Distortion Map section. Right and left translations routinely show the most severe distortions in the sections falling between $30-150^{\circ}$. Clockwise and counter-clockwise torque show the most severe distortion in the parallel ridges falling between 210 and $330^{\circ}$ in right slant loops and whorls. Intense values extend up into Angle 150 and 330 of plain arches as there is a more horizontal flow of ridges in these regions for arches. Up translation follows the same trends as torque movements, with some severe distortion in the extreme upper regions between $30-150^{\circ}$

Planar glass impression produces limited distortion throughout the fingerprints, rarely displacing minutiae more than $0.70 \mathrm{~mm}$. Down translation produced relatively low distortion throughout the fingerprint. Maximum displacement of minutiae is typically observed in the extreme upper or lower regions of the fingerprint. The large difference in displacement of minutiae by down translation in relation to the other movements can possibly be attributed to the overall construct of the finger. For left, right, up, and torque distortions movement is directed about the sides of the distal phalanges only. When translated downward, the movement is directed along the length of all three phalanges of the finger. This may limit the actual distance the finger can move before slipping in addition to what appears to be limited flexibility of the skin in the upper region of the fingerprint area. 


\begin{tabular}{cccc}
\multicolumn{4}{c}{ Maximum Euclidean Distance by Angle Section } \\
\hline Angle & Loops (mm) & Arches (mm) & Whorls (mm) \\
\hline 30 & 2.6 (Left) & $2.4(\mathrm{CW})$ & $1.8(\mathrm{CCW})$ \\
90 & 2.0 (Left) & $2.8($ Down) & $3.3(\mathrm{CW})$ \\
150 & 2.4 (Right) & $1.4(\mathrm{CCW})$ & $2.7(\mathrm{Up})$ \\
210 & $2.3(\mathrm{CCW})$ & $3.4(\mathrm{CW})$ & $3.3(\mathrm{CW})$ \\
270 & $3.0(\mathrm{CW})$ & $3.9(\mathrm{CW})$ & $3.6(\mathrm{CW})$ \\
330 & $2.7(\mathrm{CW})$ & $2.7(\mathrm{CW})$ & 2.6 (Right) \\
\hline
\end{tabular}

Table 5. Maximum Euclidean distances for each angle section. $\mathrm{CW}$ and $\mathrm{CCW}$ represent clockwise and counterclockwise torque. All others refer to translation of the finger

Maximum Euclidean Distance By Distortion

\begin{tabular}{lccc}
\hline \multicolumn{1}{c}{ Distortion } & Loop $(\mathrm{mm})$ & Arches $(\mathrm{mm})$ & Whorls $(\mathrm{mm})$ \\
\hline Translation Left & 2.4 & 2.6 & 3.2 \\
Translation Right & 2.6 & 2.7 & 3.4 \\
Translation Up & 2.4 & 2.2 & 2.9 \\
Translation Down & 2.0 & 2.8 & 2.1 \\
Torque Counter- & 2.3 & 2.5 & 2.8 \\
Clockwise & & & \\
Torque Clockwise & 3.0 & 3.9 & 3.6 \\
Planar Glass & 1.3 & 2.4 & 2.0 \\
\hline
\end{tabular}

Table 6. Maximum Euclidean distances observed for minutiae displaced by each distortion condition.

Finger Type

A range of fingers on the hand were used because the accessible population limited the ability to target a specific finger on the hand. Five Thumbs, 21 index fingers, 2 middle fingers, and 2 ring fingers were studied. The limits of variability arising from distortion were analyzed for each of the finger types. Index fingers exhibit a global Euclidean mean of $.51 \mathrm{~mm}$, thumbs a mean of $0.62 \mathrm{~mm}$, middle fingers a mean of $0.52 \mathrm{~mm}$, and ring fingers a mean of $0.31 \mathrm{~mm}$. ANCOVA and a 95\% confidence Tukey test indicate that index and middle fingers are indistinguishable from each other. All other fingers can be distinguished from each other. The mean values for each finger by radius are shown in Table 7.

The index, thumbs, and middle fingers exhibit similar amounts of variability; all three have similar Euclidean means up to a radius of $8 \mathrm{~mm}$ (Figure 24). At $9 \mathrm{~mm}$, middle finger minutiae experience on average $0.13 \mathrm{~mm}$ less displacement than index and thumbs, and at 10 $\mathrm{mm}$ middle finger minutiae experience more than $0.30 \mathrm{~mm}$ less displacement on average. It is not clear if the middle finger differences at Radius 9 and 10 are true differences or if it is due to a small sample size of 2 middle fingers in the study. The index and middle fingers differ by a maximum of $0.050 \mathrm{~mm}$ at all other radial bins resulting in indistinguishable means. 
Mean Euclidean Distance of Distorted Minutiae by Finger Type

\begin{tabular}{ccccccccc}
\hline Radius & $\begin{array}{c}\text { Index } \\
(\mathrm{mm})\end{array}$ & s.d. & $\begin{array}{c}\text { Thumb } \\
(\mathrm{mm})\end{array}$ & s.d. & $\begin{array}{c}\text { Middle } \\
(\mathrm{mm})\end{array}$ & s.d & $\begin{array}{c}\text { Ring } \\
(\mathrm{mm})\end{array}$ & s.d. \\
\hline 0.001 & 0.13 & 0.089 & 0.13 & 0.068 & 0.099 & 0.067 & ----- & ----- \\
1 & 0.20 & 0.12 & 0.22 & 0.15 & 0.17 & 0.091 & 0.15 & 0.017 \\
2 & 0.27 & 0.17 & 0.31 & 0.19 & 0.32 & 0.16 & 0.22 & 0.022 \\
3 & 0.35 & 0.25 & 0.40 & 0.26 & 0.38 & 0.22 & 0.27 & 0.026 \\
4 & 0.44 & 0.30 & 0.47 & 0.27 & 0.41 & 0.26 & 0.30 & 0.023 \\
5 & 0.51 & 0.35 & 0.52 & 0.34 & 0.58 & 0.35 & 0.38 & 0.029 \\
6 & 0.60 & 0.41 & 0.65 & 0.36 & 0.60 & 0.30 & 0.49 & 0.10 \\
7 & 0.70 & 0.47 & 0.74 & 0.40 & 0.74 & 0.47 & ---- & ----- \\
8 & 0.75 & 0.48 & 0.76 & 0.44 & 0.78 & 0.56 & ----- & ----- \\
9 & 0.82 & 0.53 & 0.85 & 0.43 & 0.69 & 0.32 & ----- & ----- \\
10 & 0.95 & 0.55 & 1.05 & 0.46 & 0.68 & 0.22 & ----- & ----- \\
\hline
\end{tabular}

Table 7. Mean Euclidean values of each finger type at each radius section. Values are reported with their standard deviations. ----- indicates no minutiae were present in the radius section. A total of 9,785 minutiae observations were made for thumbs, 31,036 for index fingers, 3,869 for middle fingers, and 867 for ring fingers. The observations are broken down by radial section in Appendix A.

Minutiae were not present in all of the radial bins for the ring fingers studied, but the sections containing minutiae displayed noticeably smaller Euclidean means. ANCOVA and a 95\% Tukey test indicate that ring finger means are indistinguishable from the other fingers at Radius 1,2,3, and 6. The ring finger means are distinguishable from the other fingers at Radius 4 and 5 and just thumbs at Radius 3 . The Euclidean mean of ring finger minutiae differed by as much as $0.20 \mathrm{~mm}$ at these radii. More specifically, ring finger means were distinguishable at Angle 90 and indistinguishable at all other Angle sections for Radius 4. In Radius 5, the ring finger means were only distinguishable at Angles 90 and 270.

As with middle fingers, only 2 ring fingers were used in this study. These small sample sizes make it difficult to conclude if there are considerable differences in the range of variability across fingers of the hand. The results of this study suggest there to be little to no difference in the variability of index, thumb, and middle fingers up to Radius 8 , and the variability observed in ring fingers will be significantly less than the other three. Clearly, more replicates of each finger need to be studied for definitive conclusions to be drawn. 


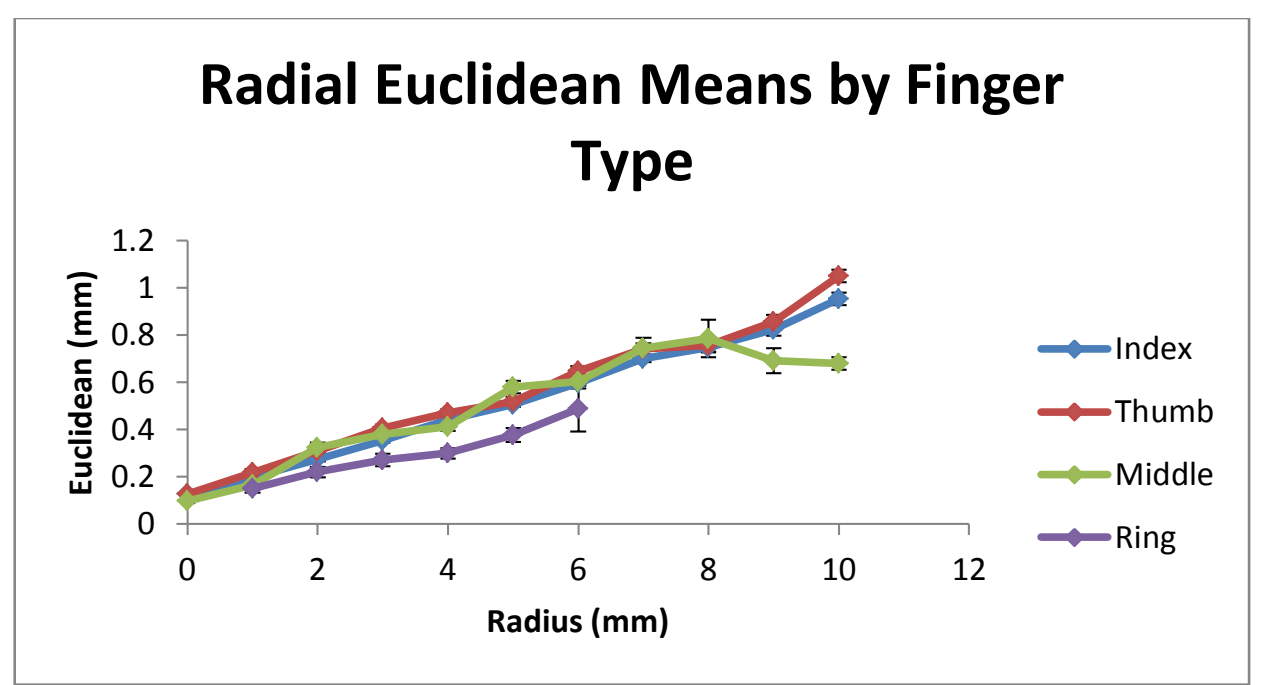

Figure 24. Euclidean means of distorted minutiae for the finger types studied. Mean values are represented with 95\% confidence. The number of observation at each Radius can be found in Appendix A.

Gender

Of the 27 individuals studied 16 were male and 11 were female. Males exhibit a global Euclidean mean of $0.54 \mathrm{~mm}$ and Females exhibit a global mean of $0.53 \mathrm{~mm}$. This initially suggest limited to no difference between the two. The variability observed in each radial bin is closely similar as well. Some slight differences are observable from Radius 4 to Radius 7 (Figure 25). ANCOVA and a 95\% Tukey test indicate significant differences between males and females at Radius 4, 5, 6, 7, and 10.

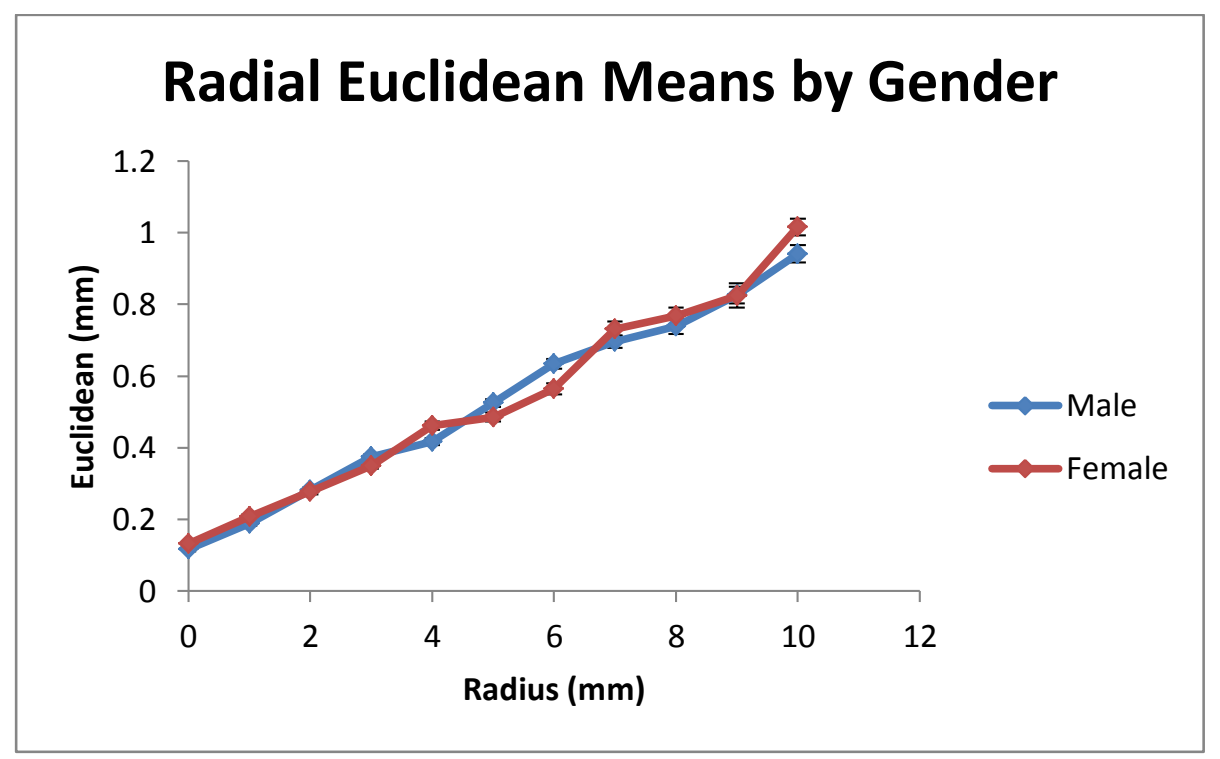

Figure 25. Mean Euclidean values for Males and Females at each radius section. Means are presented with $95 \%$ confidence. The number of minutiae observations per Radius can be seen in Table 8 .

While statistically distinguishable, the two means never differ by more than $0.074 \mathrm{~mm}$. This difference is small enough to where there would be approximately a 3 pixel difference in 
variability between the two. Based on these observations there appears to be no practical difference between male and female fingerprint variability. Matching systems would not be able to differentiate the two well, and $0.074 \mathrm{~mm}$ is likely indistinguishable by the human eye. The mean Euclidean values for each radius section are shown in Table 8.

Mean Euclidean Distance of Distorted Minutiae by Gender

\begin{tabular}{ccccccc}
\hline Radius & Male $(\mathrm{mm})$ & s.d. & Observations & Female $(\mathrm{mm})$ & s.d. & Observations \\
\hline 0.001 & 0.12 & 0.075 & 708 & 0.13 & 0.091 & 579 \\
1 & 0.19 & 0.13 & 1828 & 0.21 & 0.12 & 1407 \\
2 & 0.28 & 0.18 & 2252 & 0.28 & 0.16 & 1310 \\
3 & 0.37 & 0.27 & 3333 & 0.35 & 0.21 & 2145 \\
4 & 0.42 & 0.28 & 3516 & 0.46 & 0.31 & 2532 \\
5 & 0.53 & 0.37 & 4354 & 0.49 & 0.30 & 2271 \\
6 & 0.63 & 0.40 & 3374 & 0.57 & 0.36 & 2132 \\
7 & 0.70 & 0.45 & 2484 & 0.73 & 0.46 & 2149 \\
8 & 0.74 & 0.49 & 2095 & 0.77 & 0.44 & 1419 \\
9 & 0.83 & 0.51 & 1804 & 0.83 & 0.47 & 773 \\
10 & 0.94 & 0.55 & 1992 & 1.0 & 0.39 & 1100 \\
\hline
\end{tabular}

Table 8. Mean Euclidean values of males and females at each radius section. Values are reported their standard deviations.

Weight Loss

One of the subjects participating in the study (plain whorl) reported a weight loss of approximately 100 pounds over the last year and a half. Weight loss of this kind, in a relatively short period of time, can lead to the occurrence of loose skin. The variability in the subject's finger was isolated and compared to the complete finger and whorl populations. The mean Euclidean values for this individual fell within the expected variability for both whorls and the complete finger population. The largest Euclidean distance observed for each of the 7 distortion conditions were not responsible for any of the maximum values observed in the entire study population. While there was no apparent difference in variability for this individual, extreme weight loss over a very short period of time may have more noticeable effects on the variability of an individual's fingerprints.

\section{Orientation}

The change in minutiae orientation was examined for 120 (4 from each finger) of the minutiae examined in this study. Change in orientation resulting from distortions was analyzed across all distortion replicate movements for the entire set of minutiae, for ending ridges and bifurcations, and for static and displaced minutiae. Static minutiae were defined as minutiae displaced by $0.10 \mathrm{~mm}$ or less, and displaced minutiae are those which were displaced by more than $0.10 \mathrm{~mm}$.

Variability in minutiae orientation does not follow the same radial property as observed with minutiae location. Maximum variability is observed in Radius 3-6 with variability 
decreasing as minutiae approach the core or edge of the fingerprint. The largest mean change in orientation occurs in Radius 5 at $9.1^{\circ}$, and the smallest mean change occurs in Radius 001 at $3.1^{\circ}$ (Table 9). An orientation change of $33^{\circ}$ was the largest single change in minutiae orientation observed for any of the minutiae studied. This change was observed in Radius 5, Angle 270 under clockwise torque. A global mean of $5.4^{\circ}$ is observed for all minutiae examined.

\begin{tabular}{cccc}
\multicolumn{4}{c}{ Variability of Minutiae Orientation } \\
\hline Radius & $\begin{array}{c}\text { Mean Orientation } \\
\text { Change }\left({ }^{\circ}\right)\end{array}$ & s.d. & Observations \\
\hline 0.001 & 3.1 & 3.3 & 500 \\
1 & 3.6 & 3.6 & 595 \\
2 & 5.6 & 4.9 & 420 \\
3 & 6.6 & 5.6 & 630 \\
4 & 6.0 & 4.6 & 630 \\
5 & 9.1 & 6.5 & 385 \\
6 & 6.1 & 4.2 & 280 \\
7 & 5.4 & 4.4 & 175 \\
8 & 5.3 & 4.5 & 280 \\
9 & 4.7 & 4.0 & 140 \\
10 & 3.8 & 3.0 & 105 \\
\hline
\end{tabular}

Table 9. Change in orientation of minutiae by radius section. Values presented with their standard deviations.

There appears to be a difference in the mean orientation changes between static and displaced minutiae. A larger mean orientation is seen for displaced minutiae at all radii except for Radius 9 (Table 10). Displaced minutiae values differ by as much as $3.9^{\circ}$. Displaced minutiae also exhibit a global mean of $7.2^{\circ}$ while static minutiae have a global mean of $4.8^{\circ}$. ANCOVA and a 95\% Tukey test indicate an overall difference between static and displaced minutiae and a difference in means at Radius 1 and Radius 4-8. This difference is expected, as a larger shift in location will likely force a larger change in orientation. More than 74 percent of the static minutiae have a radius less than $5 \mathrm{~mm}$. Close proximity to the core has been shown to exhibit less variability in minutiae location and explains why there is less variability in minutiae orientation for static minutiae. 
Variability in Static and Displaced Minutiae Orientaion

\begin{tabular}{ccccccc}
\hline Radius & Static $\left(^{\circ}\right)$ & s.d. & Observations & Displaced $\left(^{\circ}\right)$ & s.d. & Observations \\
\hline 0.001 & 2.9 & 2.9 & 274 & 4.4 & 4.9 & 45 \\
1 & 3.1 & 3.0 & 282 & 5.2 & 4.8 & 83 \\
2 & 5.4 & 4.4 & 147 & 6.0 & 5.6 & 97 \\
3 & 6.5 & 5.6 & 286 & 7.1 & 5.8 & 86 \\
4 & 5.1 & 3.4 & 271 & 8.1 & 6.2 & 107 \\
5 & 7.6 & 4.8 & 114 & 10.5 & 7.6 & 109 \\
6 & 5.4 & 3.5 & 93 & 9.3 & 5.1 & 23 \\
7 & 4.5 & 3.8 & 57 & 8.0 & 5.1 & 19 \\
8 & 4.5 & 3.1 & 116 & 8.0 & 7.1 & 33 \\
9 & 6.0 & 4.1 & 24 & 3.5 & 3.5 & 27 \\
10 & 3.4 & 3.0 & 32 & 5.2 & 3.0 & 10 \\
\hline
\end{tabular}

Table 10. Mean change in orientation for minutiae deemed static or displaced. Values presented with their standard deviations.

The variability of ending ridge orientation and bifurcation orientation were compared as well. No bifurcations were observed in Radius 6-10. This makes it difficult to determine if there is a true difference between the two minutiae types throughout the entire fingerprint. Bifurcations experienced an average of $5.2^{\circ}$ change in orientation and ending ridges experienced an average of 5.6 . ANCOVA and a 95\% Tukey test indicated the two are indistinguishable at Radius 001, 1,2 , and 4 . The two are distinguishable at Radius 3 and Radius 5. Ending ridges experience an average of $3.1^{\circ}$ greater change in orientation than bifurcations at Radius 5, but $2.2^{\circ}$ less at Radius 3 (Table 11).

Variability in Minutiae Orientation by Type

\begin{tabular}{ccccccc}
\hline Radius & Ending Ridge $\left({ }^{\circ}\right)$ & sd & Observations & Bifurcation $\left({ }^{\circ}\right)$ & sd & Observations \\
\hline 0.001 & 3.5 & 2.7 & 100 & 2.9 & 3.5 & 219 \\
1 & 4.2 & 3.9 & 215 & 2.7 & 2.8 & 150 \\
2 & 6.0 & 4.4 & 103 & 5.4 & 5.2 & 141 \\
3 & 5.3 & 3.8 & 148 & 7.5 & 6.4 & 224 \\
4 & 5.6 & 3.4 & 224 & 6.5 & 5.8 & 154 \\
5 & 9.9 & 6.3 & 161 & 6.8 & 6.5 & 62 \\
6 & 6.1 & 4.2 & 116 & ---- & ---- & \\
7 & 5.4 & 4.4 & 76 & ---- & ---- & \\
8 & 5.3 & 4.5 & 149 & ---- & ----- & \\
9 & 4.7 & 4.0 & 51 & ---- & ----- & \\
10 & 3.8 & 3.0 & 42 & ---- & ----- & \\
\hline
\end{tabular}

Table 11. Mean change in orientation for ending ridges and bifurcations at each radius, presented with standard deviations. ----- indicates no minutiae were observed in the radius section.

The maximum change in orientation for bifurcations and ending ridges are 33 and $29^{\circ}$ respectively. It is difficult to say if there is a true difference in the variability of ending ridge and bifurcation orientations, but each has the potential to be altered by as much as $30^{\circ}$. 
Clockwise and counter-clockwise torque created maximum orientation changes of 25$32^{\circ}$. These extremes were most commonly observed for minutiae in the lower parallel flowing ridges that had orientations close to $180^{\circ}$ or $0^{\circ}$. Left and right translations created maximum orientation changes of $23-29^{\circ}$. These extremes were observed for minutiae falling in Angle 30 and Angle $90\left(30-150^{\circ}\right)$ of the finger. Up and down translations created maximum orientation changes of $20-25^{\circ}$. These extremes were located throughout the fingerprint area in minutiae with orientations around $45,135,225$, or $315^{\circ}$. As expected planar glass impression created the least rotational change with maximum values of $11-14^{\circ}$. These extremes were observed for minutiae in the lower parallel flowing ridges. As with the change in minutiae location, it appears as if distortions directed mostly perpendicular to the minutiae orientation or surrounding ridge flow creates the largest changes in minutiae orientation.

\section{Distortion Maps}

The frequency of Euclidean distances was analyzed for the entire dataset (Appendix C). A distance of 0-0.7 $\mathrm{mm}$ accounted for over $73 \%$ of the total distances observed. This data was roughly divided in half by increments of $0-0.3 \mathrm{~mm}$ and $0.3-0.7 \mathrm{~mm}$. Furthermore, a bounding box size of radius 15 pixels was proposed [2] for systems not employing elastic registration of fingerprints. The pixel ratio in the distorted images was approximately 38 pixels per $\mathrm{mm}$, meaning distances of 0-0.3 mm would fall within a conservatively sized elastic registration bounding box of radius 12 pixels. Distances of $0.3-0.7 \mathrm{~mm}$ would fall within an area of about twice the size of these bounding boxes. The remaining data was divided into groups of $0.7-1 \mathrm{~mm}$ and distances greater than $1 \mathrm{~mm}$ based on their relative frequencies. These intervals divided the remaining data approximately in half.

Categorical values were assigned to each group, describing the amount of variability in minutiae location. Minimal refers to Euclidean distances of 0-0.3 mm (approx. 0-12 pixels); these minutiae fall within bounding boxes with minimal registration of the fingerprints required. Moderate refers to Euclidean distances of 0.3-0.7 mm (approx. 12-27 pixels); these minutiae require slightly more of a registration to align two fingerprints. Significant refers to Euclidean distances of 0.7-1 mm (approx. 27-38 pixels); displacements of these magnitudes require large amounts of a registration to align two fingerprints. Severe refers to displacements of larger than 1 $\mathrm{mm}$, these minutiae have a greater chance of non-matches due to their severe displacements.

Each section was colored based on its mean Euclidean value. Sections where no minutiae were observed were colored based on the mean of the radius of which the section falls. Areas with the most sections containing no observed minutiae were typically Angles 150 and 330 at Radius 9 and 10. Maps entitled with just a pattern type indicate the cumulative effects of all 7 distortions. 

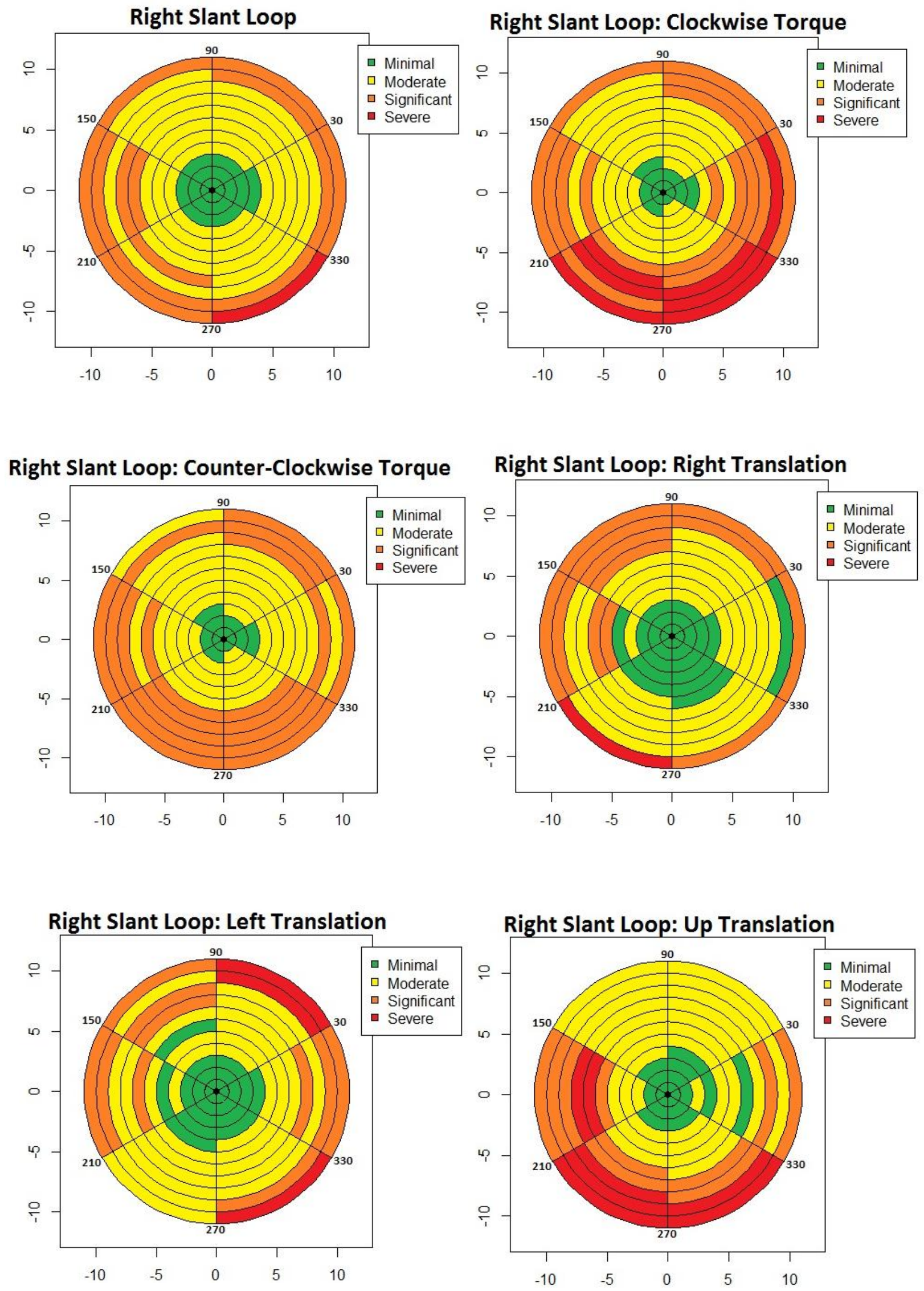
Right Slant Loop: Down Translation
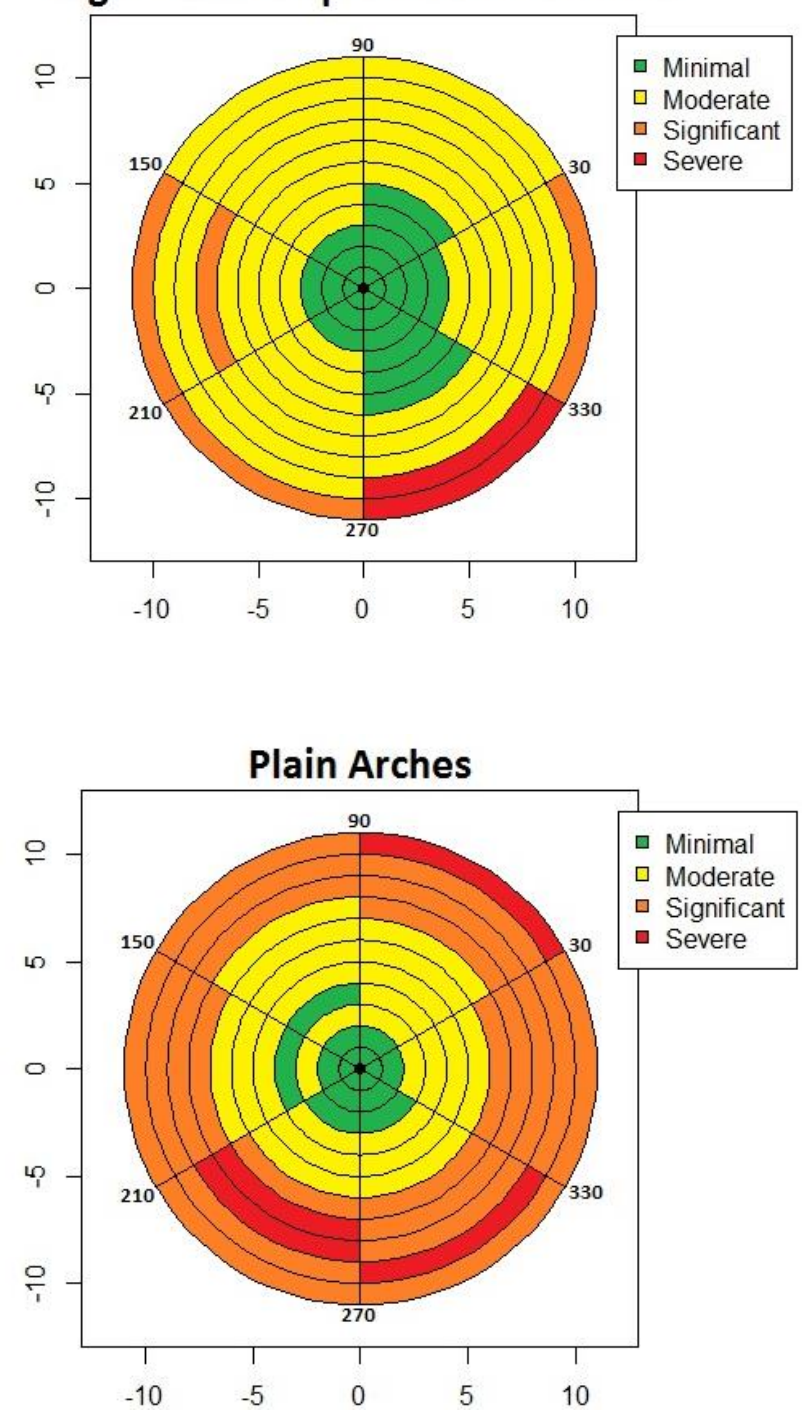

Plain Arch: Counter-Clockwise Torque

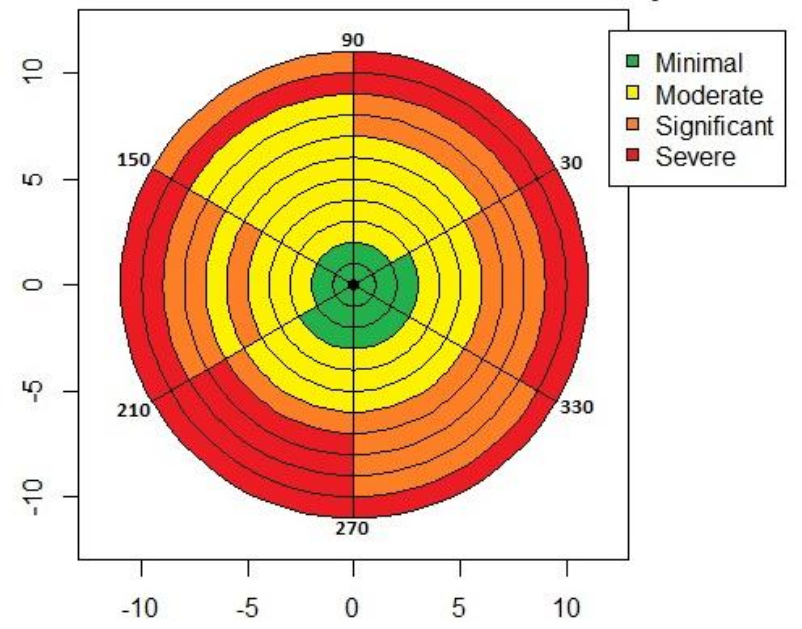

Right Slant Loop: Planar Glass

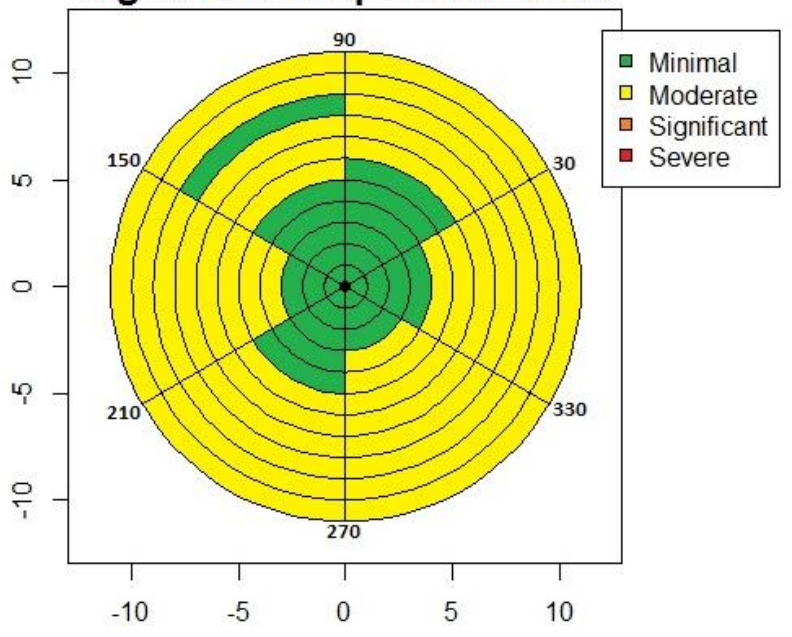

Plain Arch: Clockwise Torque

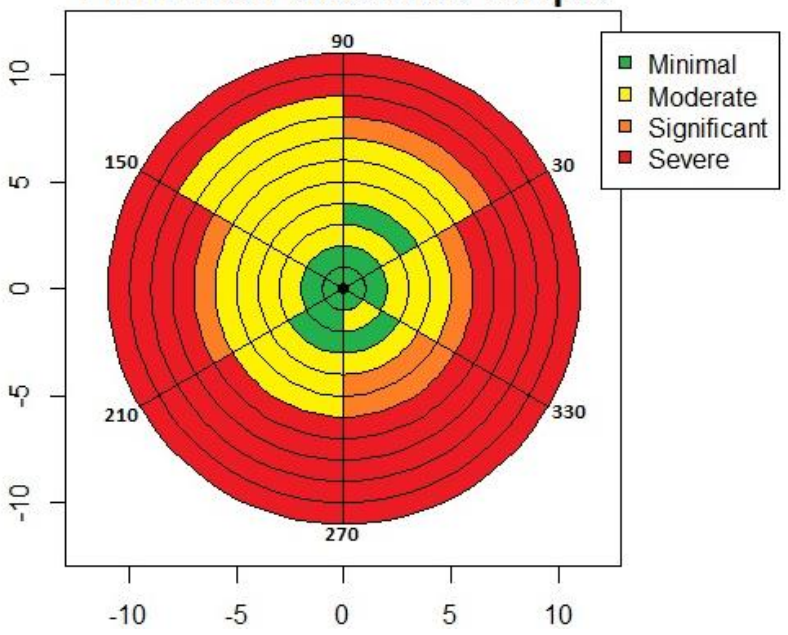

Plain Arch: Right Translation

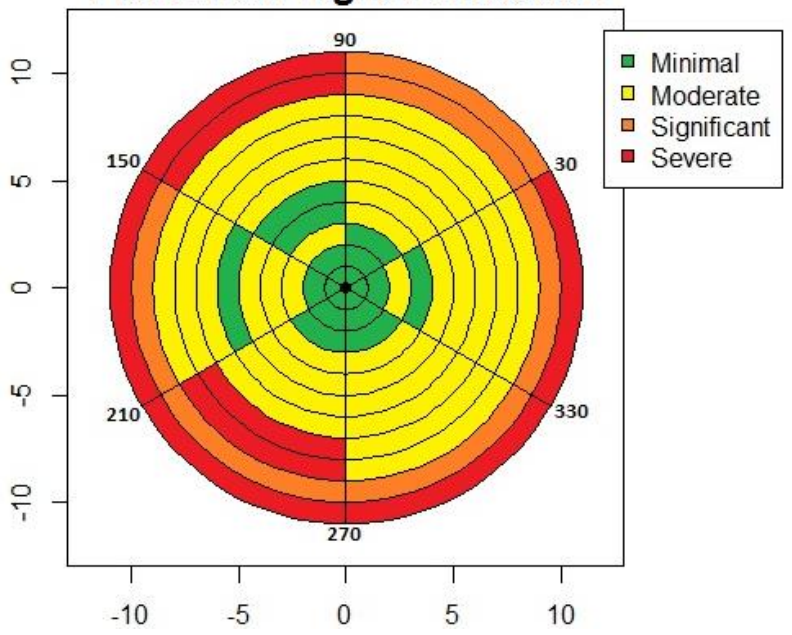



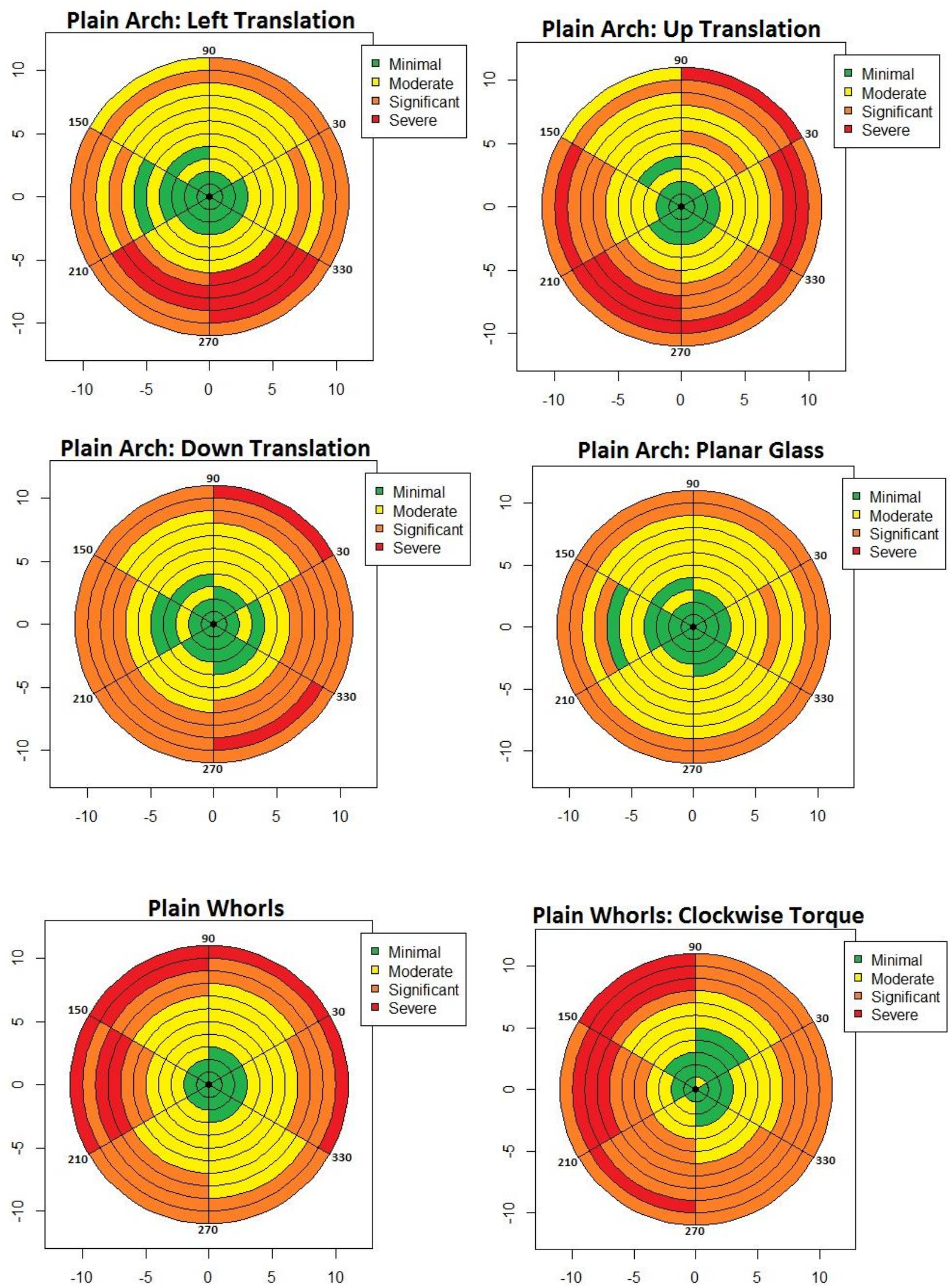
Plain Whorls: Counter-Clockwise Torque
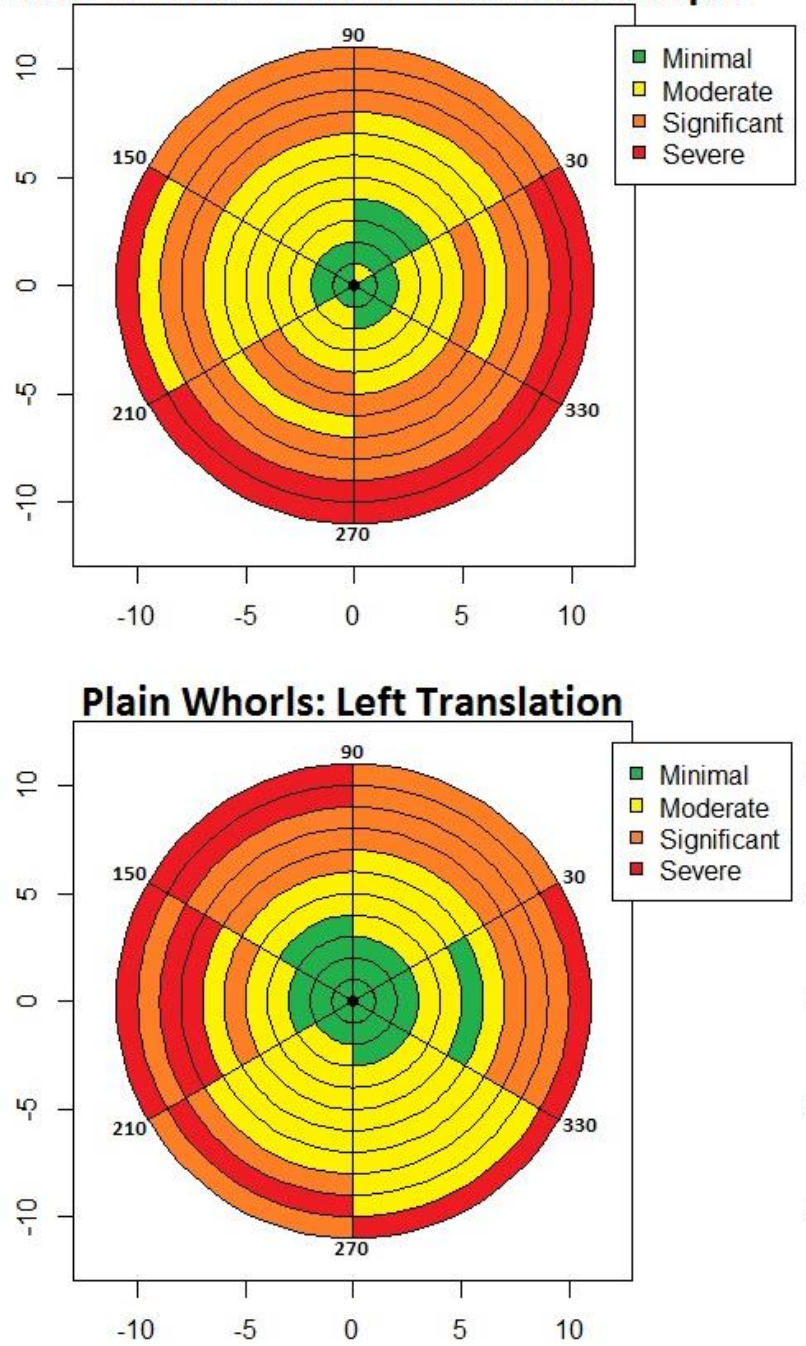

Plain Whorl: Down Translation

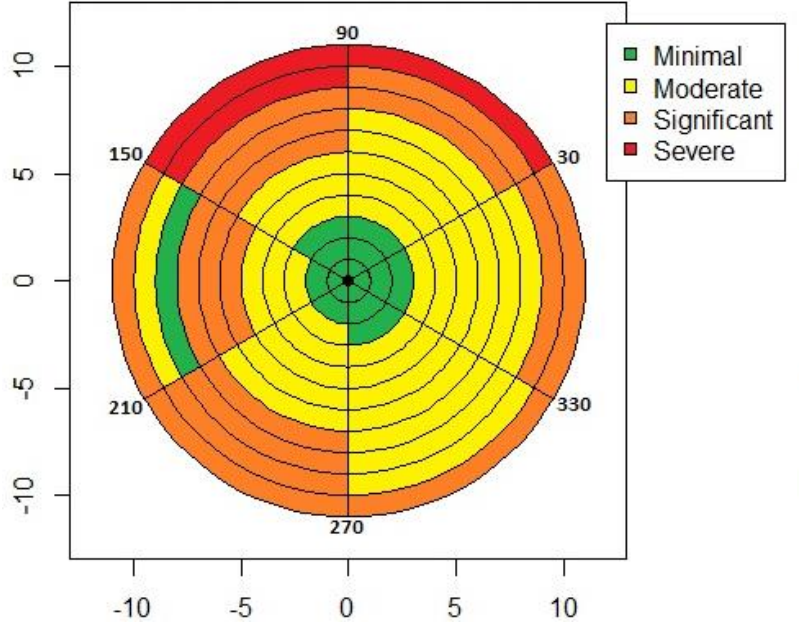

Plain Whorls: Right Translation

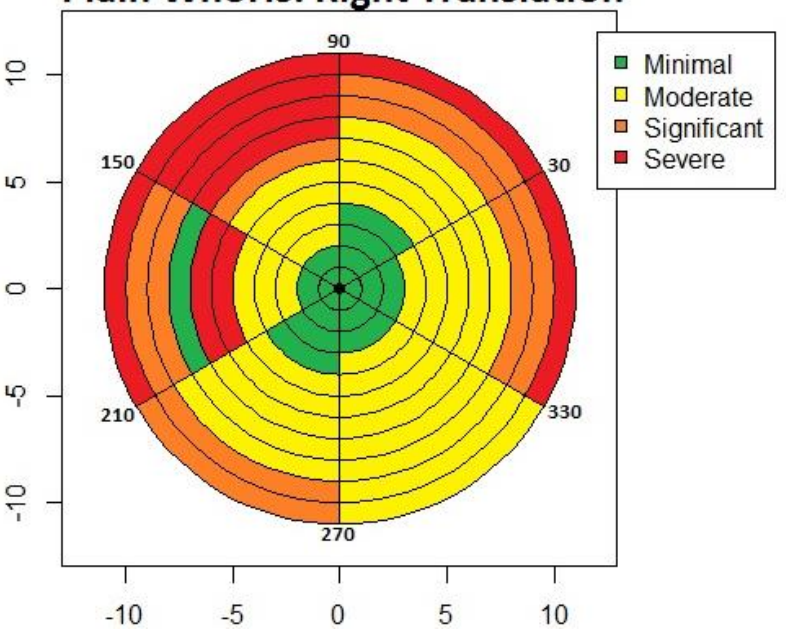

Plain Whorls: Up Translation

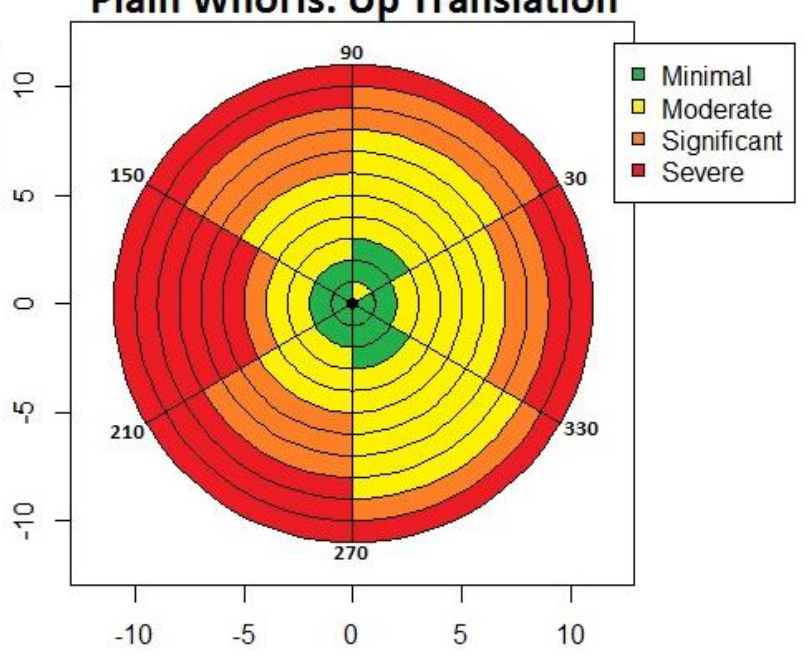

Plain Whorls: Planar Glass

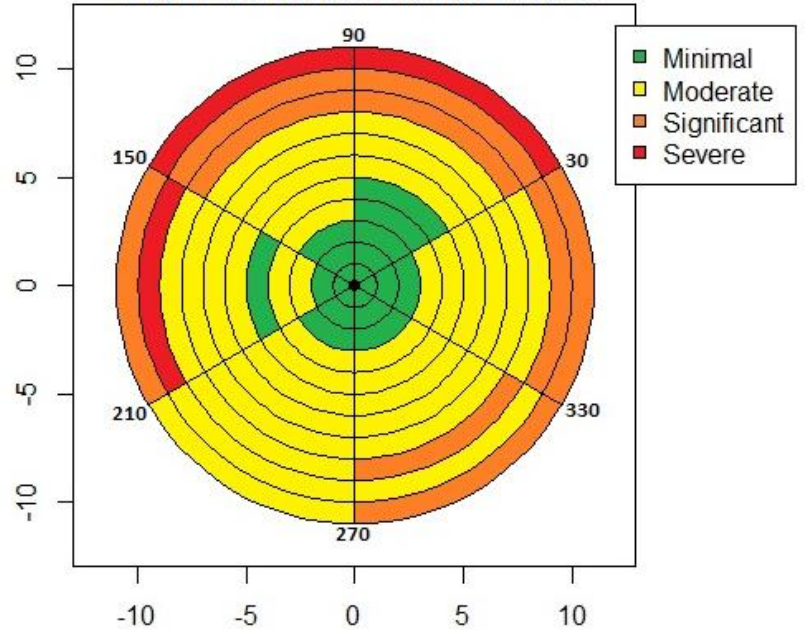



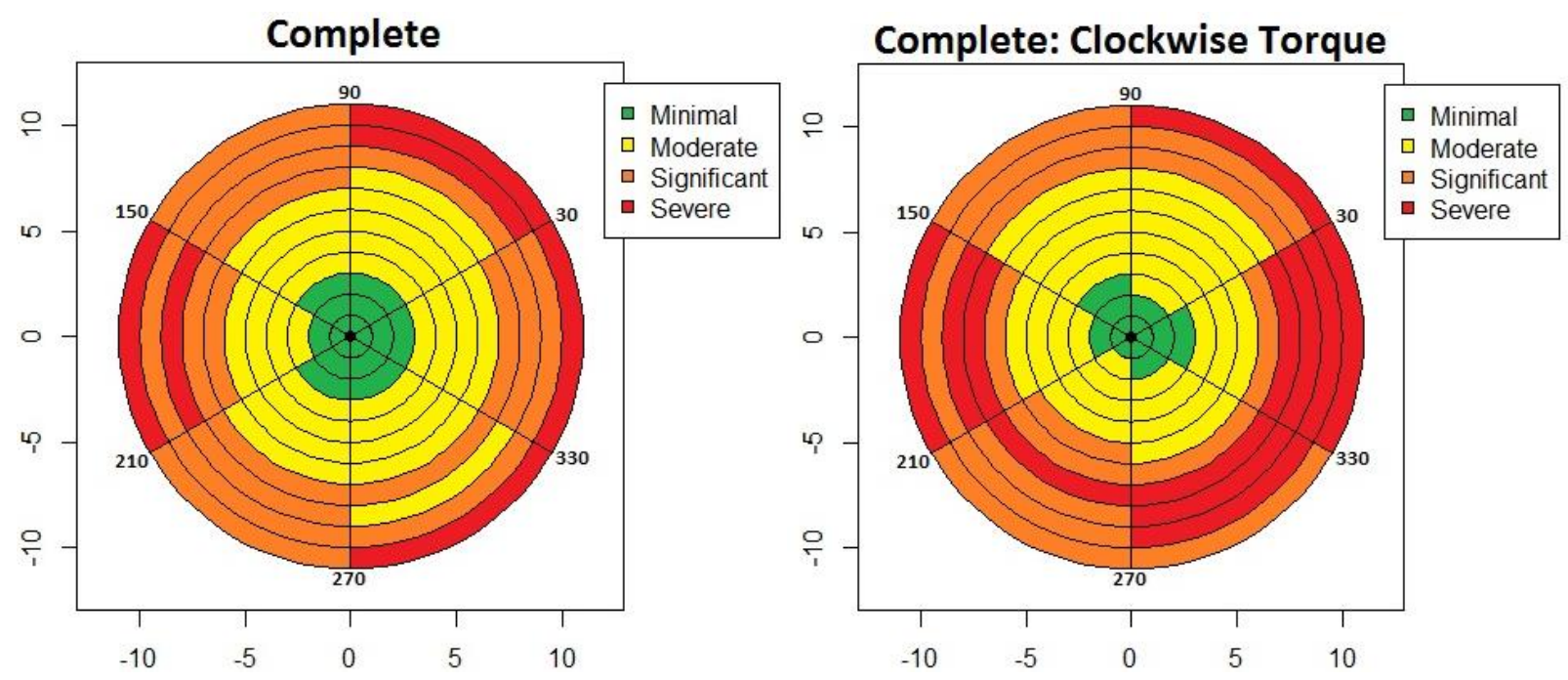

Complete: Counter-Clockwise Torque
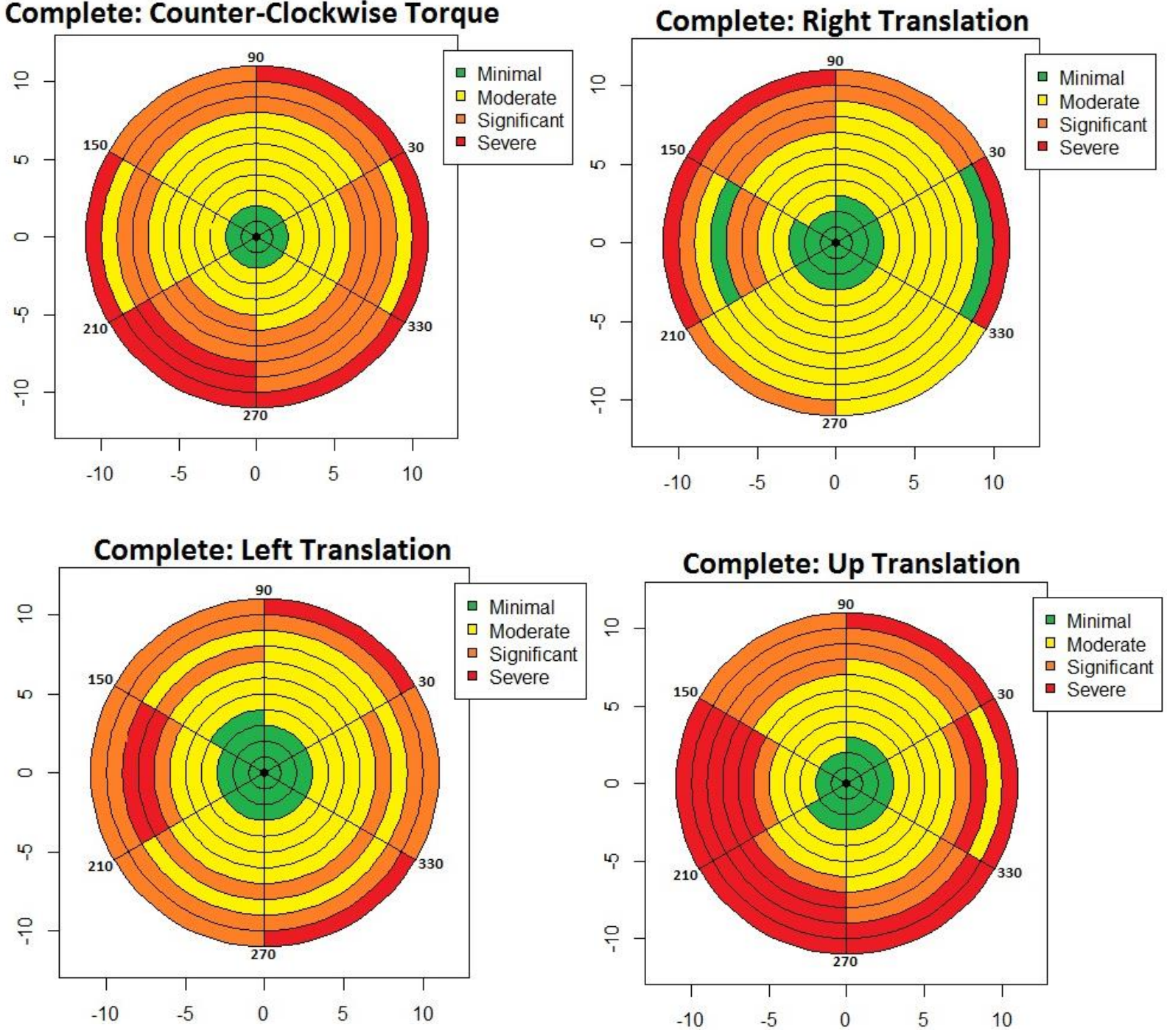

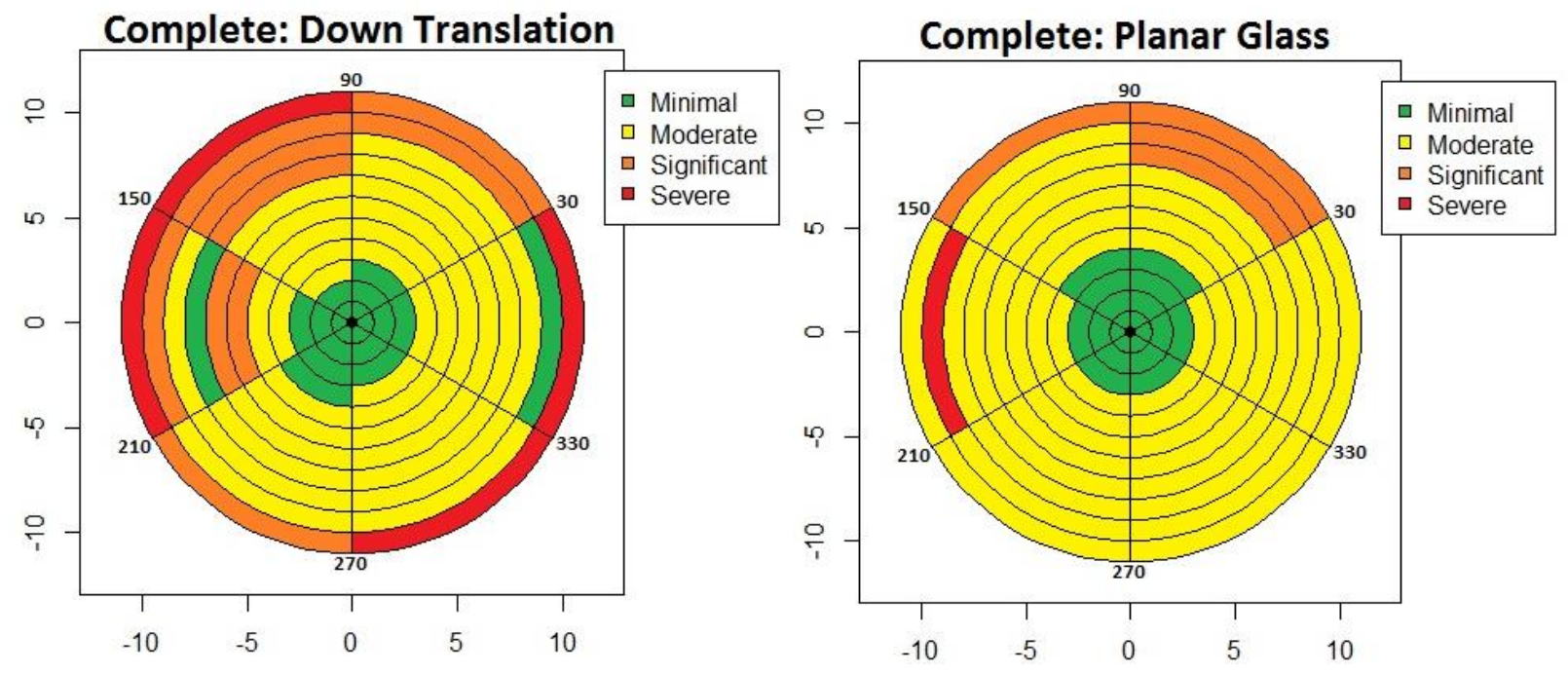

Test of Model

A set of latent finger prints was collected from subject 23 to tests the limits of variability in actual latent impressions. The latent prints were deposited on the same piece of glass the fingerprint videos were recorded. Prints were deposited with a heavy deposition pressure and various movements of the finger were performed without sliding the finger across the glass. The prints were then developed with black fingerprint powder and brush, and were photographed along with the same NIST ruler used in the distortion videos. The images were then opened in ImageJ and the scale was set, minutiae marked, and coordinates recorded in the same manner as the inked planar and distorted images. A total of 9 latent impressions were collected.

The mean and maximum Euclidean distances were recorded for the latent set at each radius section and compared to the complete set of fingers and right slant loops (Table 12). The mean values for the latent fingerprints were slightly larger than right slant loops and the complete set at all but Radius .001 and Radius 10. The maximum displacement observed at each radius falls well within the maximum values observed for both right slant loops and the complete set. While the mean values were slightly higher they fall within the natural variation observed, and the maximum displacements we noticeably small than the entire dataset.

Even though a heavier deposition pressure and deliberate movements were implemented in the collection of latent prints, the latent prints are of relatively high quality. Two of the latent impressions are shown in Figure 26. The ability to accurately mark minutiae locations decreases with the quality of latent fingerprints. Latent prints recovered from crime scenes are often of lesser quality and rarely contain the entire fingerprint. This can make it difficult to accurately measure the amount of variability present in latent prints. 
Mean and Maximum displacement of Minutiae Location

\begin{tabular}{rcccccc}
\hline Radius & $\begin{array}{c}\text { Latent Mean } \\
(\mathrm{mm})\end{array}$ & $\begin{array}{c}\text { Latent Max } \\
(\mathrm{mm})\end{array}$ & $\begin{array}{c}\text { Loop Mean } \\
(\mathrm{mm})\end{array}$ & $\begin{array}{c}\text { Loop Max } \\
(\mathrm{mm})\end{array}$ & $\begin{array}{c}\text { Complete } \\
\text { Mean }(\mathrm{mm})\end{array}$ & $\begin{array}{c}\text { Complete } \\
\text { Max }(\mathrm{mm})\end{array}$ \\
\hline 0.001 & 0.12 & 0.23 & 0.10 & 0.26 & 0.12 & 0.48 \\
1 & 0.22 & 0.50 & 0.18 & 0.71 & 0.20 & 1.5 \\
2 & 0.28 & 0.85 & 0.26 & 1.1 & 0.28 & 1.1 \\
3 & 0.52 & 1.3 & 0.37 & 2.1 & 0.36 & 2.1 \\
4 & 0.64 & 1.5 & 0.39 & 1.7 & 0.44 & 2.4 \\
5 & 0.63 & 1.7 & 0.46 & 2.4 & 0.51 & 2.6 \\
6 & 0.70 & 2.1 & 0.59 & 2.8 & 0.61 & 3.0 \\
7 & 0.76 & 2.0 & 0.63 & 2.7 & 0.71 & 3.4 \\
8 & 0.95 & 2.5 & 0.66 & 3.0 & 0.75 & 3.3 \\
9 & 1.2 & 2.3 & 0.70 & 2.7 & 0.83 & 3.9 \\
10 & 0.87 & 2.0 & 0.77 & 2.6 & 0.97 & 3.6 \\
\hline
\end{tabular}

Table 12. Mean and maximum minutiae displacement for minutiae observed in latent fingerprints from subject 23 , right slant loops, and complete finger set. A total of 399 minutiae observations were made in the set of latent impressions.

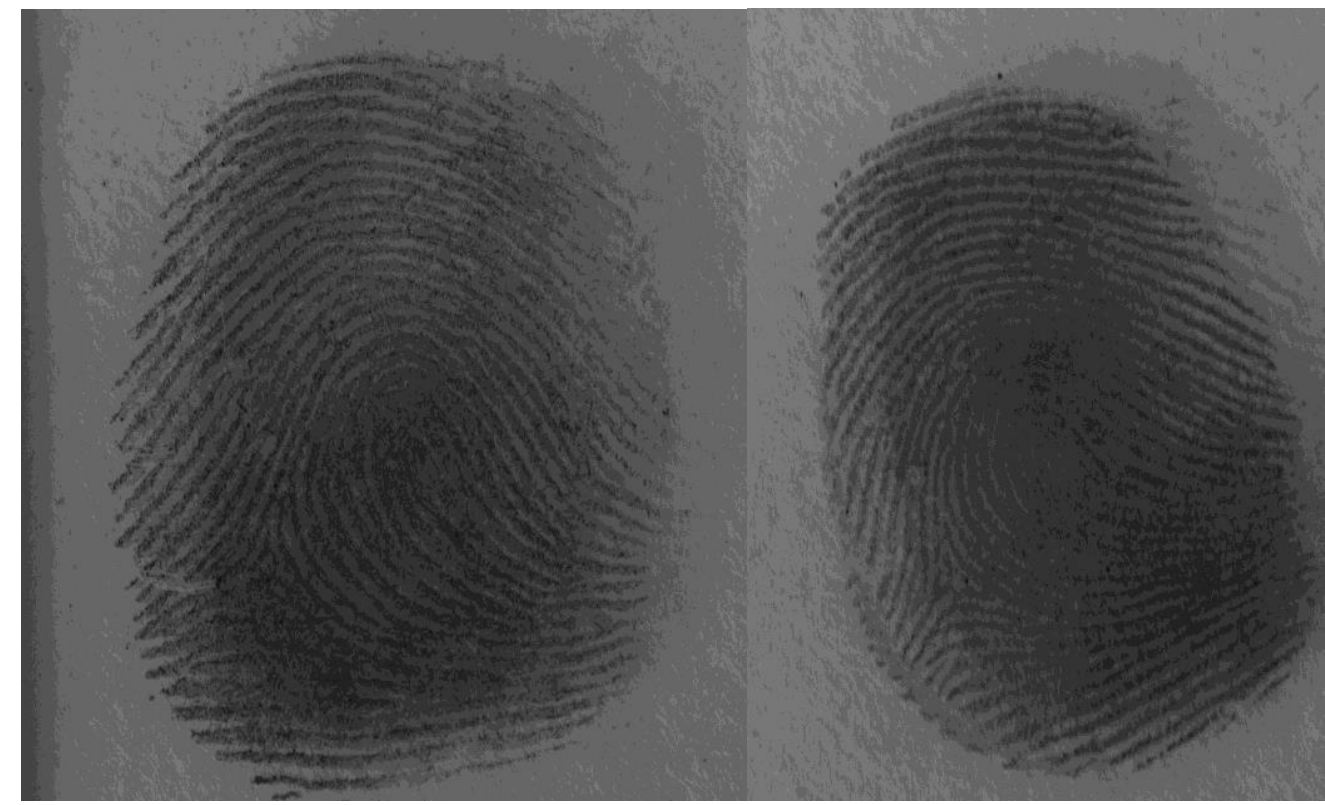

Figure 26. Two of the latent prints collected from subject 23 .

\section{Practical Applicability}

The findings of this study can be applied directly to the daily analysis of fingerprint examiners. First, this study has identified absolute maximums in variability, and the expected amounts of variability under heavy distortion throughout the fingerprint region. With these 
findings examiners can numerically define variability of minutiae location and orientation arising from distortion. The distortion maps will provide examiners with a tool to describe what is an acceptable distortion when testifying in court.

Furthermore, the distortion maps can help examiners to understand the amount of variability expected in the various regions of fingerprints and how different distortions affect the various areas of a fingerprint. The report of the National Academy of Science [1] addresses the need for documentation of the steps performed in fingerprint comparisons by examiners. The results of this study in conjunction with documentation of distortions observed by the examiner in fingerprint comparisons will strengthen the conclusions drawn for the comparison of two prints.

The limits of variability can also be incorporated into the design and development of automated matching systems. Matching systems perform some type of registration to align fingerprints in the matching process. By identifying the limits of variability, a limit can be set to the amount of registration used in the alignment process. The trends in minutiae displacement can also be used in the construction of a bounding box system. A variable sized bounding box similar to that proposed by $\mathrm{He}[6]$ appears to be an ideal system for determining minutiae matching pairs.

\section{Conclusion}

This study has directly addressed the concerns raised by the 2009 NAS report. First, variability has been studied across a range of repeated impressions of a single finger. Inked planar impressions for an individual can be expected to exhibit an average of $0.18 \mathrm{~mm}$ of variability in minutiae location for any given minutiae in the fingerprint. Variability in location larger than $0.5 \mathrm{~mm}$ is rarely observed, and discrepancies larger than this would likely indicate the presence of two different donors of the impressions being compared.

Secondly, variability has been characterized, quantified, and compared across a population of fingers consisting of right slant loops, plain arches, and plain whorls. Minutiae can be displaced by more than $3 \mathrm{~mm}$ and their orientation altered by up to $30^{\circ}$ when subject to heavy distortion. Displacements of $1 \mathrm{~mm}$ and rotations of $10^{\circ}$ are commonly observed for minutiae when significant distortion is applied. This degree of variability can produce largely overlapping regions for minutiae in close proximity, creating difficulties for both examiners and matching systems.

The amount of variability in minutiae location and orientation both reach their maximums in areas where the direction of distortion is perpendicular to the ridge flow. There also appears to be an increased flexibility of the skin in the lower regions of the fingerprint. Minutiae falling in the parallel flowing ridges of this area often exhibit the most variability of minutiae in fingerprints. Also, minutiae in right slant loops appear to be less susceptible to distortion than both plain arches and plain whorls.

Torque movements routinely create the most variability in minutiae location, creating upwards of $3 \mathrm{~mm}$ of displacement in the lower parallel ridges of fingerprints. Left and right translations of the finger tend to impart greater variability in the areas above the core region; 
displacing minutiae by more than $2 \mathrm{~mm}$ in these areas. While an up translation of the finger can impart a great deal of variability throughout the fingerprint, down translations produce a minimal amount of variability in relation to the other distortions.

Finally, the results of this study will provide fingerprint examiners with a tool to both quantitatively and qualitatively describe an acceptable distortion. The distortion maps developed can be implemented to explicitly define the amounts of variability that can be reasonably expected in the various regions of fingerprints. The directionality of displacement has also been observed for various distortion conditions. The use of distortion maps and the characterization of distortion types can be used in conjunction with examiner documentation of comparisons to increase the strength in the identification or exclusion of fingerprints.

This study has laid the ground work for a more complete understanding of the limits of fingerprint variability. Analysis of a second set of fingerprints under the same set of conditions would support the findings of this study. More replicates of each finger of the hand should be studied to better understand the limits of fingerprint variability in each finger as well. The study can also be expanded to encompass the various loop, arch, whorl, and accidental pattern types not studied here. Once variability is understood across pattern types and fingers of the hand, it should be studied in depth for latent fingerprints. Latent fingerprints present additional factors which can affect fingerprint variability.

To conclude, several distinct trends have been observed for fingerprint variability:

- There is a clear radial property to fingerprint variability; it increases as radius increases.

- A greater flexibility of skin is observed in the lower parallel flowing ridges of the finger

- Inked planar impressions exhibit minimal variability with a global mean of $0.176 \mathrm{~mm}$

- Distortion produces the most variability when directed perpendicular to ridge flow

- Minutiae can be displaced by extremes of $3 \mathrm{~mm}$ and orientation alter by up to $30^{\circ}$ from heavy distortion

- Displacements of $1 \mathrm{~mm}$ and orientation changes of $10^{\circ}$ more commonly observed

- Torque or up translation of the finger produce the most amount of variability in fingerprints

- Down translations of the finger produces minimal variability in fingerprints; similar in magnitude to planar glass impressions

- There appears to be no difference in variability between males and females

- Variability appears to be the same for thumbs, index, and middle fingers, with less variability observed in ring fingers 


\section{References}

[1] Strengthening Forensic Science in The United States: A Path Forward.; The National Academies Press: Washington, D. C., 2009.

[2] Bazen, A. M.; Gerez, S. H. Fingerprint Matching by Thin-plate Spline Modeling of Elastic Deformations. Pattern Recognition. 2002, 36, 1857-1867.

[3] Cappelli, R.; Maio, D.; Maltoni, D. Modeling Plastic Distortion in Fingerprint Images, Proceedings of the Second International Conference on Advances in Pattern Recognition (ICAPR 2001), Rio de Janeiro, March 2001, pp. 369-376.

[4] Maltoni, D.; Cappelli, R., Advances in Fingerprint Modeling. Image and Vision Computing. 2009, 27, 258-268.

[5] Jain, A., Hong, L., \& Bolle, R. On-line fingerprint verification. IEEE Transactions on Pattern Analysis and Machine Intelligence. 1997, 19(4), 302-314.

[6] He, Y.; Tian, J.; Luo, X.; Zhang, T., Image Enhancement and Minutiae Matching in Fingerprint Verification. Pattern Recognition Letters. 2003, 24, 1394-1360.

[7] Senior, A.; Bolle, R. Improved Fingerprint Matching by Distortion Removal. IEICE Transactions on Information and Systems. 2001, 54, 825-831.

[8] Maceo, A. V. Qualitative Assessment of Skin Deformation: A Pilot Study. Journal of Forensic Identification. 2009, 59, 390-440.

[9] Nikon D7000 User's Manual. Nikon Corporation, Tokyo, Japan.

[10] McKillup, S. Statistics Explained: An Introductory Guide for Life Sciences,; Cambridge University Press: Ney York, 2005.

[11] Siegle, A; Morgan, C. Statistics and Data Analysis: An Introduction, $2^{\text {nd }}$ ed.; John Wily \& Sons, INC.: New York, 1996 


\section{Appendix A}

Distortion by Radius

Mean and Maximum Euclidean Distances of Right Distorted Minutiae

\begin{tabular}{ccccccc}
\hline Radius & $\begin{array}{c}\text { Loop Mean } \\
(\mathrm{mm})\end{array}$ & $\begin{array}{c}\text { Loop Max } \\
(\mathrm{mm})\end{array}$ & $\begin{array}{c}\text { Arch Mean } \\
(\mathrm{mm})\end{array}$ & $\begin{array}{c}\text { Arch Max } \\
(\mathrm{mm})\end{array}$ & $\begin{array}{c}\text { Whorl Mean } \\
(\mathrm{mm})\end{array}$ & $\begin{array}{c}\text { Whorl Max } \\
(\mathrm{mm})\end{array}$ \\
\hline 0.001 & 0.10 & 0.26 & 0.13 & 0.32 & 0.13 & 0.36 \\
1 & 0.17 & 0.63 & 0.18 & 0.57 & 0.20 & 0.59 \\
2 & 0.20 & 0.89 & 0.26 & 0.55 & 0.27 & 0.74 \\
3 & 0.32 & 2.1 & 0.32 & 0.82 & 0.34 & 1.7 \\
4 & 0.34 & 1.6 & 0.34 & 1.1 & 0.43 & 2.0 \\
5 & 0.41 & 2.4 & 0.45 & 1.3 & 0.47 & 2.6 \\
6 & 0.52 & 2.3 & 0.52 & 1.1 & 0.56 & 1.9 \\
7 & 0.55 & 2.0 & 0.56 & 1.4 & 0.63 & 2.1 \\
8 & 0.52 & 1.5 & 0.61 & 1.5 & 0.71 & 2.4 \\
9 & 0.73 & 2.0 & 0.75 & 1.6 & 0.77 & 2.2 \\
10 & 0.89 & 1.89 & 1.1 & 2.6 & 1.2 & 3.2 \\
\hline
\end{tabular}

Mean and Maximum Euclidean Distances of Left Distorted Minutiae

\begin{tabular}{ccccccc}
\hline Radius & $\begin{array}{c}\text { Loop Mean } \\
(\mathrm{mm})\end{array}$ & $\begin{array}{c}\text { Loop Max } \\
(\mathrm{mm})\end{array}$ & $\begin{array}{c}\text { Arch Mean } \\
(\mathrm{mm})\end{array}$ & $\begin{array}{c}\text { Arch Max } \\
(\mathrm{mm})\end{array}$ & $\begin{array}{c}\text { Whorl Mean } \\
(\mathrm{mm})\end{array}$ & $\begin{array}{c}\text { Whorl Max } \\
(\mathrm{mm})\end{array}$ \\
\hline 0.001 & 0.098 & 0.25 & 0.15 & 0.39 & 0.12 & 0.28 \\
1 & 0.18 & 0.65 & 0.20 & 0.60 & 0.20 & 0.57 \\
2 & 0.20 & 0.75 & 0.28 & 0.70 & 0.27 & 0.71 \\
3 & 0.34 & 2.0 & 0.35 & 1.2 & 0.36 & 1.4 \\
4 & 0.33 & 1.5 & 0.43 & 1.7 & 0.45 & 1.5 \\
5 & 0.37 & 2.2 & 0.53 & 1.4 & 0.51 & 1.9 \\
6 & 0.56 & 2.0 & 0.65 & 2.3 & 0.62 & 1.9 \\
7 & 0.65 & 1.7 & 0.87 & 2.7 & 0.73 & 1.8 \\
8 & 0.56 & 1.7 & 0.68 & 2.2 & 0.75 & 2.4 \\
9 & 0.70 & 2.1 & 0.87 & 2.7 & 0.89 & 2.7 \\
10 & 0.92 & 2.7 & 0.82 & 1.6 & 1.1 & 3.4 \\
\hline
\end{tabular}


Mean and Maximum Euclidean Distances of Up Distorted Minutiae

\begin{tabular}{ccccccc}
\hline Radius & $\begin{array}{c}\text { Loop Mean } \\
(\mathrm{mm})\end{array}$ & $\begin{array}{c}\text { Loop Max } \\
(\mathrm{mm})\end{array}$ & $\begin{array}{c}\text { Arch Mean } \\
(\mathrm{mm})\end{array}$ & $\begin{array}{c}\text { Arch Max } \\
(\mathrm{mm})\end{array}$ & $\begin{array}{c}\text { Whorl Mean } \\
(\mathrm{mm})\end{array}$ & $\begin{array}{c}\text { Whorl Max } \\
(\mathrm{mm})\end{array}$ \\
\hline 0.001 & 0.11 & 0.23 & 0.15 & 0.45 & 0.14 & 0.40 \\
1 & 0.19 & 0.69 & 0.20 & 0.51 & 0.21 & 0.52 \\
2 & 0.27 & 0.76 & 0.30 & 0.89 & 0.31 & 0.82 \\
3 & 0.38 & 1.5 & 0.35 & 1.1 & 0.37 & 1.2 \\
4 & 0.41 & 1.5 & 0.48 & 1.8 & 0.56 & 1.7 \\
5 & 0.55 & 2.2 & 0.59 & 1.5 & 0.59 & 2.3 \\
6 & 0.69 & 2.4 & 0.61 & 1.4 & 0.70 & 1.8 \\
7 & 0.71 & 2.0 & 0.74 & 2.2 & 0.89 & 2.7 \\
8 & 0.78 & 2.0 & 0.82 & 2.0 & 1.0 & 2.8 \\
9 & 0.75 & 2.1 & 1.0 & 2.1 & 1.1 & 2.9 \\
10 & 0.77 & 2.1 & 0.92 & 2.2 & 1.3 & 2.7 \\
\hline
\end{tabular}

Mean and Maximum Euclidean Distances of Down Distorted Minutiae

\begin{tabular}{ccccccc}
\hline Radius & $\begin{array}{c}\text { Loop Mean } \\
(\mathrm{mm})\end{array}$ & $\begin{array}{c}\text { Loop Max } \\
(\mathrm{mm})\end{array}$ & $\begin{array}{c}\text { Arch Mean } \\
(\mathrm{mm})\end{array}$ & $\begin{array}{c}\text { Arch Max } \\
(\mathrm{mm})\end{array}$ & $\begin{array}{c}\text { Whorl Mean } \\
(\mathrm{mm})\end{array}$ & $\begin{array}{c}\text { Whorl Max } \\
(\mathrm{mm})\end{array}$ \\
\hline 0.001 & 0.10 & 0.23 & 0.16 & 0.48 & 0.12 & 0.36 \\
1 & 0.16 & 0.46 & 0.18 & 0.52 & 0.19 & 0.58 \\
2 & 0.24 & 0.75 & 0.27 & 0.63 & 0.28 & 0.77 \\
3 & 0.31 & 1.2 & 0.27 & 0.69 & 0.36 & 0.96 \\
4 & 0.32 & 1.1 & 0.39 & 1.2 & 0.45 & 1.2 \\
5 & 0.38 & 1.1 & 0.49 & 1.3 & 0.51 & 1.6 \\
6 & 0.51 & 1.2 & 0.56 & 1.3 & 0.60 & 1.9 \\
7 & 0.52 & 1.5 & 0.75 & 1.7 & 0.69 & 1.9 \\
8 & 0.49 & 1.0 & 0.74 & 1.6 & 0.68 & 2.0 \\
9 & 0.61 & 2.0 & 0.93 & 1.6 & 0.80 & 1.7 \\
10 & 0.70 & 1.5 & 0.90 & 2.8 & 0.92 & 2.1 \\
\hline
\end{tabular}


Mean and Maximum Euclidean Distances of Counter-Clockwise Torque Distorted Minutiae

\begin{tabular}{ccccccc}
\hline Radius & $\begin{array}{c}\text { Loop Mean } \\
(\mathrm{mm})\end{array}$ & $\begin{array}{c}\text { Loop Max } \\
(\mathrm{mm})\end{array}$ & $\begin{array}{c}\text { Arch Mean } \\
(\mathrm{mm})\end{array}$ & $\begin{array}{c}\text { Arch Max } \\
(\mathrm{mm})\end{array}$ & $\begin{array}{c}\text { Whorl Mean } \\
(\mathrm{mm})\end{array}$ & $\begin{array}{c}\text { Whorl Max } \\
(\mathrm{mm})\end{array}$ \\
\hline 0.001 & 0.11 & 0.26 & 0.19 & 0.44 & 0.14 & 0.37 \\
1 & 0.21 & 0.70 & 0.19 & 0.57 & 0.25 & 0.93 \\
2 & 0.36 & 1.0 & 0.32 & 0.83 & 0.38 & 1.1 \\
3 & 0.48 & 1.3 & 0.36 & 0.96 & 0.46 & 1.9 \\
4 & 0.50 & 1.5 & 0.43 & 1.2 & 0.58 & 2.0 \\
5 & 0.60 & 1.8 & 0.58 & 1.6 & 0.64 & 1.8 \\
6 & 0.71 & 2.0 & 0.68 & 1.8 & 0.64 & 2.0 \\
7 & 0.70 & 2.2 & 0.83 & 2.0 & 0.78 & 2.4 \\
8 & 0.77 & 2.3 & 0.92 & 2.5 & 0.90 & 2.7 \\
9 & 0.78 & 1.6 & 1.1 & 1.7 & 1.0 & 2.8 \\
10 & 0.74 & 1.8 & 1.0 & 2.2 & 1.1 & 2.4 \\
\hline
\end{tabular}

Mean and Maximum Euclidean Distances of Clockwise Torque Distorted Minutiae

\begin{tabular}{ccccccc}
\hline Radius & $\begin{array}{c}\text { Loop Mean } \\
(\mathrm{mm})\end{array}$ & $\begin{array}{c}\text { Loop Max } \\
(\mathrm{mm})\end{array}$ & $\begin{array}{c}\text { Arch Mean } \\
(\mathrm{mm})\end{array}$ & $\begin{array}{c}\text { Arch Max } \\
(\mathrm{mm})\end{array}$ & $\begin{array}{c}\text { Whorl Mean } \\
(\mathrm{mm})\end{array}$ & $\begin{array}{c}\text { Whorl Max } \\
(\mathrm{mm})\end{array}$ \\
\hline 0.001 & 0.11 & 0.24 & 0.13 & 0.41 & 0.16 & 0.48 \\
1 & 0.20 & 0.61 & 0.22 & 0.72 & 0.25 & 1.5 \\
2 & 0.34 & 1.1 & 0.34 & 0.84 & 0.31 & 1.0 \\
3 & 0.43 & 1.2 & 0.44 & 2.0 & 0.44 & 1.7 \\
4 & 0.48 & 1.7 & 0.56 & 2.3 & 0.59 & 1.9 \\
5 & 0.58 & 2.2 & 0.60 & 1.8 & 0.68 & 2.2 \\
6 & 0.76 & 2.8 & 0.870 & 3.0 & 0.68 & 2.5 \\
7 & 0.85 & 2.7 & 1.1 & 3.4 & 0.86 & 3.0 \\
8 & 0.97 & 3.0 & 1.1 & 3.3 & 0.93 & 2.6 \\
9 & 0.91 & 2.4 & 1.3 & 3.9 & 0.95 & 3.3 \\
10 & 0.87 & 2.1 & 1.2 & 2.3 & 0.97 & 3.6 \\
\hline
\end{tabular}


Mean and Maximum Euclidean Distances of Planar Glass Distorted Minutiae

\begin{tabular}{ccccccc}
\hline Radius & $\begin{array}{c}\text { Loop Mean } \\
(\mathrm{mm})\end{array}$ & $\begin{array}{c}\text { Loop Max } \\
(\mathrm{mm})\end{array}$ & $\begin{array}{c}\text { Arch Mean } \\
(\mathrm{mm})\end{array}$ & $\begin{array}{c}\text { Arch Max } \\
(\mathrm{mm})\end{array}$ & $\begin{array}{c}\text { Whorl Mean } \\
(\mathrm{mm})\end{array}$ & $\begin{array}{c}\text { Whorl Max } \\
(\mathrm{mm})\end{array}$ \\
\hline 0.001 & 0.077 & 0.18 & 0.15 & 0.38 & 0.13 & 0.31 \\
1 & 0.18 & 0.54 & 0.19 & 0.53 & 0.21 & 0.63 \\
2 & 0.21 & 0.61 & 0.25 & 0.78 & 0.27 & 0.78 \\
3 & 0.31 & 0.78 & 0.31 & 0.88 & 0.34 & 0.96 \\
4 & 0.31 & 1.0 & 0.38 & 1.2 & 0.39 & 1.1 \\
5 & 0.35 & 1.2 & 0.48 & 2.4 & 0.44 & 1.2 \\
6 & 0.40 & 1.3 & 0.52 & 1.7 & 0.50 & 1.8 \\
7 & 0.42 & 1.2 & 0.60 & 1.6 & 0.54 & 1.9 \\
8 & 0.51 & 1.3 & 0.66 & 1.8 & 0.67 & 1.7 \\
9 & 0.46 & 1.0 & 0.92 & 1.9 & 0.74 & 1.7 \\
10 & 0.53 & 1.2 & 0.89 & 2.0 & 0.96 & 2.0 \\
\hline
\end{tabular}

Distortion by Angle Section

Mean Euclidean Distance of Right Distorted Minutiae

\begin{tabular}{ccccccc}
\hline Angle & Loops $(\mathrm{mm})$ & s.d. & Arches $(\mathrm{mm})$ & s.d. & Whorls $(\mathrm{mm})$ & s.d. \\
\hline 30 & 0.51 & 0.39 & 0.53 & 0.33 & 0.56 & 0.37 \\
90 & 0.53 & 0.44 & 0.63 & 0.54 & 0.83 & 0.67 \\
150 & 0.34 & 0.42 & 0.31 & 0.20 & 0.48 & 0.41 \\
210 & 0.36 & 0.34 & 0.36 & 0.26 & 0.50 & 0.32 \\
270 & 0.35 & 0.25 & 0.38 & 0.24 & 0.40 & 0.28 \\
330 & 0.42 & 0.31 & 0.44 & 0.27 & 0.35 & 0.30 \\
\hline
\end{tabular}

Mean Euclidean Distance of Left Distorted Minutiae

\begin{tabular}{ccccccc}
\hline Angle & Loops $(\mathrm{mm})$ & s.d. & Arches $(\mathrm{mm})$ & s.d. & Whorls $(\mathrm{mm})$ & s.d. \\
\hline 30 & 0.52 & 0.46 & 0.55 & 0.31 & 0.58 & 0.36 \\
90 & 0.50 & 0.42 & 0.47 & 0.34 & 0.62 & 0.43 \\
150 & 0.39 & 0.45 & 0.31 & 0.15 & 0.52 & 0.46 \\
210 & 0.37 & 0.36 & 0.52 & 0.50 & 0.58 & 0.48 \\
270 & 0.43 & 0.38 & 0.63 & 0.55 & 0.57 & 0.47 \\
330 & 0.33 & 0.25 & 0.48 & 0.36 & 0.33 & 0.22 \\
\hline
\end{tabular}

Mean Euclidean Distance of Up Distorted Minutiae

\begin{tabular}{ccccccc}
\hline Angle & Loops $(\mathrm{mm})$ & s.d. & Arches $(\mathrm{mm})$ & s.d. & Whorls $(\mathrm{mm})$ & s.d. \\
\hline 30 & 0.41 & 0.26 & 0.70 & 0.47 & 0.61 & 0.36 \\
90 & 0.42 & 0.30 & 0.51 & 0.41 & 0.73 & 0.49 \\
150 & 0.56 & 0.47 & 0.37 & 0.21 & 0.78 & 0.63 \\
210 & 0.70 & 0.51 & 0.53 & 0.44 & 0.83 & 0.65 \\
270 & 0.53 & 0.41 & 0.50 & 0.37 & 0.58 & 0.45 \\
330 & 0.41 & 0.37 & 0.57 & 0.34 & 0.38 & 0.29 \\
\hline
\end{tabular}


Mean Euclidean Distance of Down Distorted Minutiae

\begin{tabular}{ccccccc}
\hline Angle & Loops $(\mathrm{mm})$ & s.d. & Arches $(\mathrm{mm})$ & s.d. & Whorls $(\mathrm{mm})$ & s.d. \\
\hline 30 & 0.39 & 0.23 & 0.61 & 0.38 & 0.54 & 0.33 \\
90 & 0.39 & 0.27 & 0.49 & 0.35 & 0.63 & 0.41 \\
150 & 0.38 & 0.25 & 0.27 & 0.14 & 0.54 & 0.35 \\
210 & 0.45 & 0.29 & 0.38 & 0.24 & 0.62 & 0.45 \\
270 & 0.38 & 0.33 & 0.49 & 0.34 & 0.45 & 0.31 \\
330 & 0.37 & 0.23 & 0.58 & 0.38 & 0.35 & 0.24 \\
\hline
\end{tabular}

Mean Euclidean Distance of Counter-Clockwise Torque Distorted Minutiae

\begin{tabular}{ccccccc}
\hline Angle & Loops $(\mathrm{mm})$ & s.d. & Arches $(\mathrm{mm})$ & s.d. & Whorls $(\mathrm{mm})$ & s.d. \\
\hline 30 & 0.53 & 0.31 & 0.76 & 0.42 & 0.54 & 0.34 \\
90 & 0.47 & 0.32 & 0.60 & 0.43 & 0.54 & 0.37 \\
150 & 0.46 & 0.28 & 0.40 & 0.28 & 0.54 & 0.30 \\
210 & 0.65 & 0.34 & 0.47 & 0.45 & 0.83 & 0.52 \\
270 & 0.67 & 0.44 & 0.52 & 0.36 & 0.73 & 0.51 \\
330 & 0.52 & 0.32 & 0.60 & 0.34 & 0.48 & 0.37 \\
\hline
\end{tabular}

Mean Euclidean Distance of Clockwise Torque Distorted Minutiae

\begin{tabular}{ccccccc}
\hline Angle & Loops $(\mathrm{mm})$ & s.d. & Arches $(\mathrm{mm})$ & s.d. & Whorls $(\mathrm{mm})$ & s.d. \\
\hline 30 & 0.51 & 0.30 & 0.79 & 0.46 & 0.48 & 0.32 \\
90 & 0.47 & 0.32 & 0.62 & 0.47 & 0.65 & 0.51 \\
150 & 0.47 & 0.30 & 0.37 & 0.22 & 0.68 & 0.46 \\
210 & 0.68 & 0.42 & 0.60 & 0.59 & 0.83 & 0.62 \\
270 & 0.77 & 0.58 & 0.80 & 0.68 & 0.65 & 0.54 \\
330 & 0.70 & 0.57 & 0.82 & 0.56 & 0.39 & 0.29 \\
\hline
\end{tabular}

Mean Euclidean Distance of Planar Glass Distorted Minutiae

\begin{tabular}{ccccccc}
\hline Angle & Loops $(\mathrm{mm})$ & s.d. & Arches $(\mathrm{mm})$ & s.d. & Whorls $(\mathrm{mm})$ & s.d. \\
\hline 30 & 0.31 & 0.21 & 0.61 & 0.39 & 0.54 & 0.38 \\
90 & 0.33 & 0.23 & 0.50 & 0.36 & 0.57 & 0.44 \\
150 & 0.32 & 0.22 & 0.29 & 0.18 & 0.37 & 0.25 \\
210 & 0.36 & 0.24 & 0.35 & 0.24 & 0.46 & 0.32 \\
270 & 0.42 & 0.22 & 0.42 & 0.31 & 0.50 & 0.33 \\
330 & 0.33 & 0.19 & 0.50 & 0.29 & 0.33 & 0.23 \\
\hline
\end{tabular}


Minutiae Observations by Section and Finger

Number of Minutiae Observations for Right Slant Loops by Angle Section

\begin{tabular}{cccccccc}
\hline Angle & $\begin{array}{c}\text { Right } \\
\text { Translation }\end{array}$ & $\begin{array}{c}\text { Left } \\
\text { Translation }\end{array}$ & $\begin{array}{c}\text { Up } \\
\text { Translation }\end{array}$ & $\begin{array}{c}\text { Down } \\
\text { Translation }\end{array}$ & $\begin{array}{c}\text { Clockwise } \\
\text { Torque }\end{array}$ & $\begin{array}{c}\text { Counter- } \\
\text { Clockwise Torque }\end{array}$ & $\begin{array}{c}\text { Planar } \\
\text { Glass }\end{array}$ \\
\hline 30 & 347 & 315 & 348 & 344 & 332 & 343 & 348 \\
90 & 490 & 535 & 535 & 526 & 532 & 523 & 533 \\
150 & 213 & 302 & 293 & 311 & 303 & 285 & 307 \\
210 & 483 & 579 & 548 & 564 & 569 & 546 & 552 \\
270 & 527 & 447 & 480 & 480 & 464 & 522 & 506 \\
330 & 161 & 96 & 148 & 159 & 128 & 158 & 159 \\
\hline
\end{tabular}

Number of Minutiae Observations for Plain Arches by Angle Section

\begin{tabular}{cccccccc}
\hline Angle & $\begin{array}{c}\text { Right } \\
\text { Translation }\end{array}$ & $\begin{array}{c}\text { Left } \\
\text { Translation }\end{array}$ & $\begin{array}{c}\text { Up } \\
\text { Translation }\end{array}$ & $\begin{array}{c}\text { Down } \\
\text { Translation }\end{array}$ & $\begin{array}{c}\text { Clockwise } \\
\text { Torque }\end{array}$ & $\begin{array}{c}\text { Counter- } \\
\text { Clockwise Torque }\end{array}$ & $\begin{array}{c}\text { Planar } \\
\text { Glass }\end{array}$ \\
\hline 30 & 480 & 435 & 484 & 462 & 463 & 462 & 477 \\
90 & 269 & 364 & 353 & 355 & 344 & 344 & 343 \\
150 & 51 & 94 & 83 & 88 & 90 & 85 & 92 \\
210 & 205 & 255 & 228 & 253 & 239 & 241 & 251 \\
270 & 350 & 317 & 339 & 339 & 307 & 343 & 339 \\
330 & 225 & 154 & 209 & 198 & 195 & 211 & 216 \\
\hline
\end{tabular}

Number of Minutiae Observations for Plain Whorls by Angle Section

\begin{tabular}{cccccccc}
\hline Angle & $\begin{array}{c}\text { Right } \\
\text { Translation }\end{array}$ & $\begin{array}{c}\text { Left } \\
\text { Translation }\end{array}$ & $\begin{array}{c}\text { Up } \\
\text { Translation }\end{array}$ & $\begin{array}{c}\text { Down } \\
\text { Translation }\end{array}$ & $\begin{array}{c}\text { Clockwise } \\
\text { Torque }\end{array}$ & $\begin{array}{c}\text { Counter- } \\
\text { Clockwise Torque }\end{array}$ & $\begin{array}{c}\text { Planar } \\
\text { Glass }\end{array}$ \\
\hline 30 & 363 & 315 & 360 & 343 & 341 & 343 & 359 \\
90 & 433 & 468 & 472 & 420 & 470 & 453 & 461 \\
150 & 169 & 226 & 216 & 220 & 234 & 189 & 211 \\
210 & 517 & 577 & 564 & 574 & 600 & 511 & 561 \\
270 & 700 & 649 & 675 & 704 & 645 & 678 & 697 \\
330 & 278 & 216 & 249 & 268 & 243 & 271 & 271 \\
\hline
\end{tabular}


Number of Minutiae Observations for Right Slant Loops by Radial Section

\begin{tabular}{cccccccc}
\hline Radius & $\begin{array}{c}\text { Right } \\
\text { Translation }\end{array}$ & $\begin{array}{c}\text { Left } \\
\text { Translation }\end{array}$ & $\begin{array}{c}\text { Up } \\
\text { Translation }\end{array}$ & $\begin{array}{c}\text { Down } \\
\text { Translation }\end{array}$ & $\begin{array}{c}\text { Clockwise } \\
\text { Torque }\end{array}$ & $\begin{array}{c}\text { Counter- } \\
\text { Clockwise Torque }\end{array}$ & $\begin{array}{c}\text { Planar } \\
\text { Glass }\end{array}$ \\
\hline 0.001 & 75 & 80 & 78 & 80 & 80 & 80 & 78 \\
1 & 146 & 147 & 150 & 105 & 150 & 146 & 143 \\
2 & 205 & 204 & 204 & 205 & 203 & 204 & 204 \\
3 & 266 & 261 & 257 & 264 & 260 & 267 & 260 \\
4 & 323 & 319 & 342 & 334 & 325 & 336 & 338 \\
5 & 328 & 344 & 373 & 381 & 367 & 378 & 378 \\
6 & 283 & 317 & 320 & 345 & 317 & 334 & 334 \\
7 & 191 & 183 & 195 & 198 & 204 & 197 & 212 \\
8 & 155 & 162 & 165 & 160 & 160 & 162 & 178 \\
9 & 160 & 161 & 165 & 171 & 164 & 170 & 179 \\
10 & 94 & 101 & 108 & 105 & 103 & 108 & 106 \\
\hline
\end{tabular}

Number of Minutiae Observations for Plain Arches by Radial Section

\begin{tabular}{cccccccc}
\hline Radius & $\begin{array}{c}\text { Right } \\
\text { Translation }\end{array}$ & $\begin{array}{c}\text { Left } \\
\text { Translation }\end{array}$ & $\begin{array}{c}\text { Up } \\
\text { Translation }\end{array}$ & $\begin{array}{c}\text { Down } \\
\text { Translation }\end{array}$ & $\begin{array}{c}\text { Clockwise } \\
\text { Torque }\end{array}$ & $\begin{array}{c}\text { Counter- } \\
\text { Clockwise Torque }\end{array}$ & $\begin{array}{c}\text { Planar } \\
\text { Glass }\end{array}$ \\
\hline 0.001 & 40 & 40 & 40 & 40 & 40 & 39 & 40 \\
1 & 145 & 144 & 143 & 145 & 133 & 134 & 143 \\
2 & 116 & 120 & 115 & 115 & 118 & 115 & 120 \\
3 & 221 & 239 & 235 & 239 & 226 & 236 & 238 \\
4 & 201 & 228 & 223 & 229 & 221 & 217 & 225 \\
5 & 209 & 225 & 218 & 229 & 217 & 230 & 233 \\
6 & 133 & 164 & 161 & 165 & 165 & 171 & 172 \\
7 & 185 & 148 & 193 & 176 & 187 & 198 & 192 \\
8 & 119 & 96 & 128 & 126 & 107 & 119 & 122 \\
9 & 61 & 70 & 75 & 75 & 70 & 75 & 70 \\
10 & 150 & 145 & 165 & 156 & 154 & 152 & 163 \\
\hline
\end{tabular}


Number of Minutiae Observations for Plain Whorls by Radial Section

\begin{tabular}{cccccccc}
\hline Radius & $\begin{array}{c}\text { Right } \\
\text { Translation }\end{array}$ & $\begin{array}{c}\text { Left } \\
\text { Translation }\end{array}$ & $\begin{array}{c}\text { Up } \\
\text { Translation }\end{array}$ & $\begin{array}{c}\text { Down } \\
\text { Translation }\end{array}$ & $\begin{array}{c}\text { Clockwise } \\
\text { Torque }\end{array}$ & $\begin{array}{c}\text { Counter- } \\
\text { Clockwise Torque }\end{array}$ & $\begin{array}{c}\text { Planar } \\
\text { Glass }\end{array}$ \\
\hline 0.001 & 65 & 67 & 67 & 64 & 67 & 62 & 65 \\
1 & 175 & 175 & 175 & 175 & 178 & 170 & 172 \\
2 & 190 & 188 & 188 & 187 & 191 & 184 & 186 \\
3 & 290 & 288 & 290 & 290 & 282 & 281 & 288 \\
4 & 306 & 300 & 314 & 314 & 328 & 300 & 325 \\
5 & 351 & 330 & 366 & 372 & 359 & 364 & 373 \\
6 & 316 & 281 & 299 & 302 & 302 & 305 & 320 \\
7 & 259 & 285 & 279 & 289 & 297 & 268 & 297 \\
8 & 216 & 226 & 226 & 231 & 218 & 221 & 217 \\
9 & 120 & 136 & 139 & 132 & 128 & 122 & 134 \\
10 & 177 & 180 & 198 & 178 & 188 & 173 & 188 \\
\hline
\end{tabular}

Minutiae Observations by Radius for Each Finger Type

\begin{tabular}{ccccc}
\hline Radius & Index & Thumb & Middle & Ring \\
\hline 0.001 & 908 & 239 & 140 & 0 \\
1 & 2502 & 453 & 210 & 70 \\
2 & 2549 & 699 & 209 & 105 \\
3 & 3772 & 1113 & 488 & 105 \\
4 & 4003 & 1069 & 700 & 276 \\
5 & 4521 & 1109 & 729 & 266 \\
6 & 3858 & 1237 & 366 & 45 \\
7 & 3297 & 919 & 417 & 0 \\
8 & 2391 & 929 & 194 & 0 \\
9 & 1660 & 772 & 145 & 0 \\
10 & 1575 & 1246 & 271 & 0 \\
\hline
\end{tabular}


Number of Minutiae Marked per Finger

\begin{tabular}{|c|c|c|c|c|}
\hline Subject & Template Minutiae & Distorted Minutiae & Pattern & Finger \\
\hline 1 & 45 & 43 & Right Slant & 7 \\
\hline 2 & 57 & 54 & Right Slant & 2 \\
\hline 3 & 53 & 50 & Right Slant & 2 \\
\hline 4 & 53 & 48 & Plain Whorl & 7 \\
\hline 5 & 55 & 51 & Plain Whorl & 1 \\
\hline 6 & 64 & 61 & Plain Whorl & 2 \\
\hline 7 & 43 & 41 & Plain Whorl & 7 \\
\hline 8 & 39 & 37 & Plain Arch & 2 \\
\hline 9 & 41 & 35 & Plain Whorl & 2 \\
\hline 10 & 41 & 38 & Right Slant & 2 \\
\hline 11 & 47 & 43 & Plain Whorl & 2 \\
\hline 12 & 53 & 52 & Right Slant & 1 \\
\hline 13 & 46 & 43 & Plain Arch & 2 \\
\hline 14 & 54 & 52 & Plain Whorl & 2 \\
\hline 15 & 78 & 73 & Plain Whorl & 1 \\
\hline 16 & 33 & 31 & Plain Arch & 2 \\
\hline 17 & 30 & 29 & Plain Arch & 2 \\
\hline 18 & 62 & 59 & Plain Whorl & 2 \\
\hline 19 & 53 & 47 & Right Slant & 1 \\
\hline 20 & 42 & 39 & Right Slant & 3 \\
\hline 21 & 48 & 44 & Right Slant & 3 \\
\hline 22 & 65 & 64 & Right Slant & 2 \\
\hline 23 & 50 & 47 & Right Slant & 2 \\
\hline 24 & 32 & 28 & Plain Arch & 2 \\
\hline 25 & 50 & 47 & Plain Whorl & 2 \\
\hline 26 & 67 & 62 & Plain Arch & 1 \\
\hline 27 & 27 & 26 & Plain Arch & 4 \\
\hline 28 & 28 & 27 & Plain Arch & 7 \\
\hline 29 & 32 & 31 & Plain Arch & 7 \\
\hline 30 & 31 & 29 & Plain Arch & 8 \\
\hline
\end{tabular}

**Distorted Minutiae represents the average number of "Good" quality minutiae marked in the distorted images. 
Appendix B (Variance Ellipse Charts)

Finger 1: Right Slant Loop

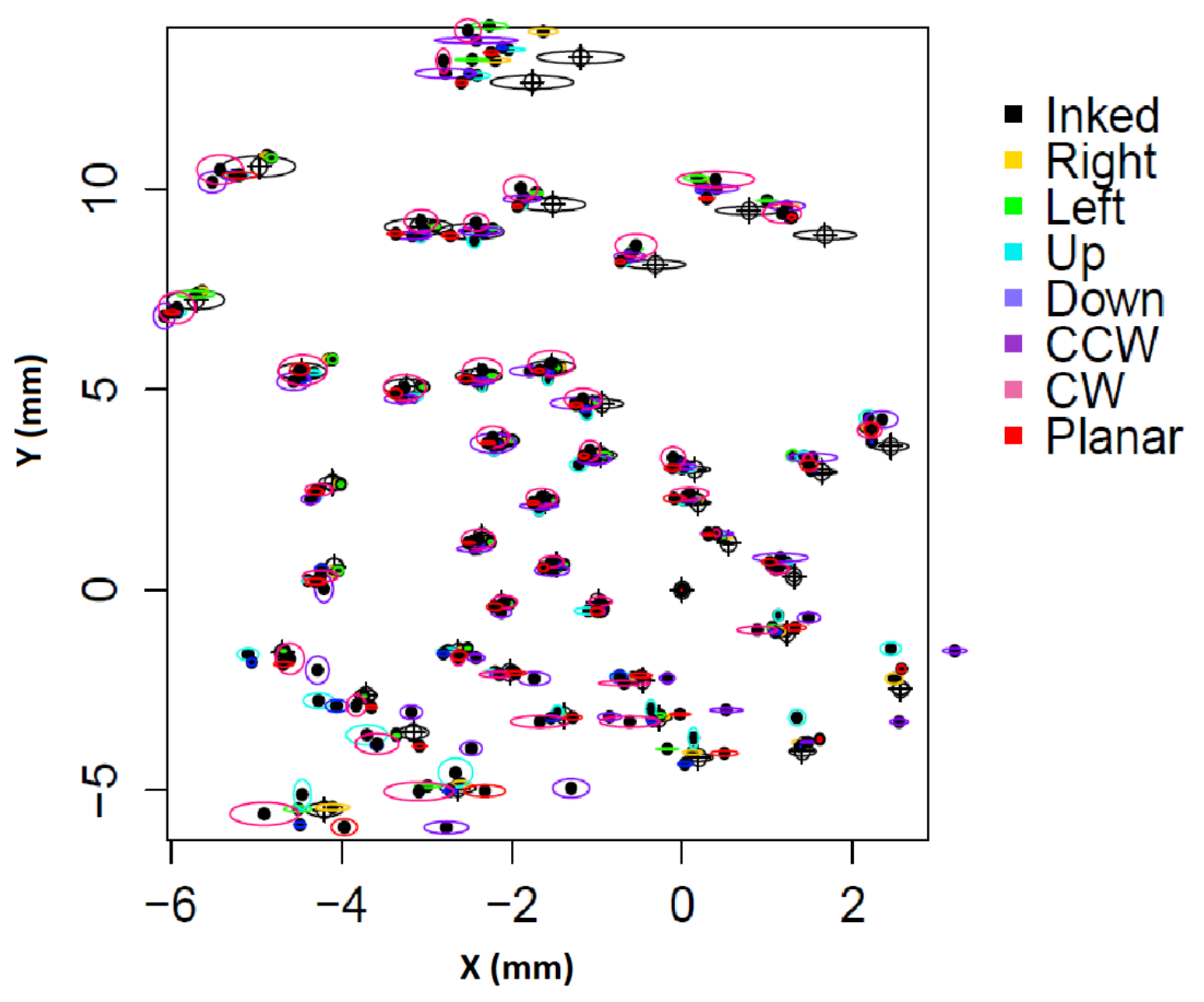


Finger 14: Plain Whorl

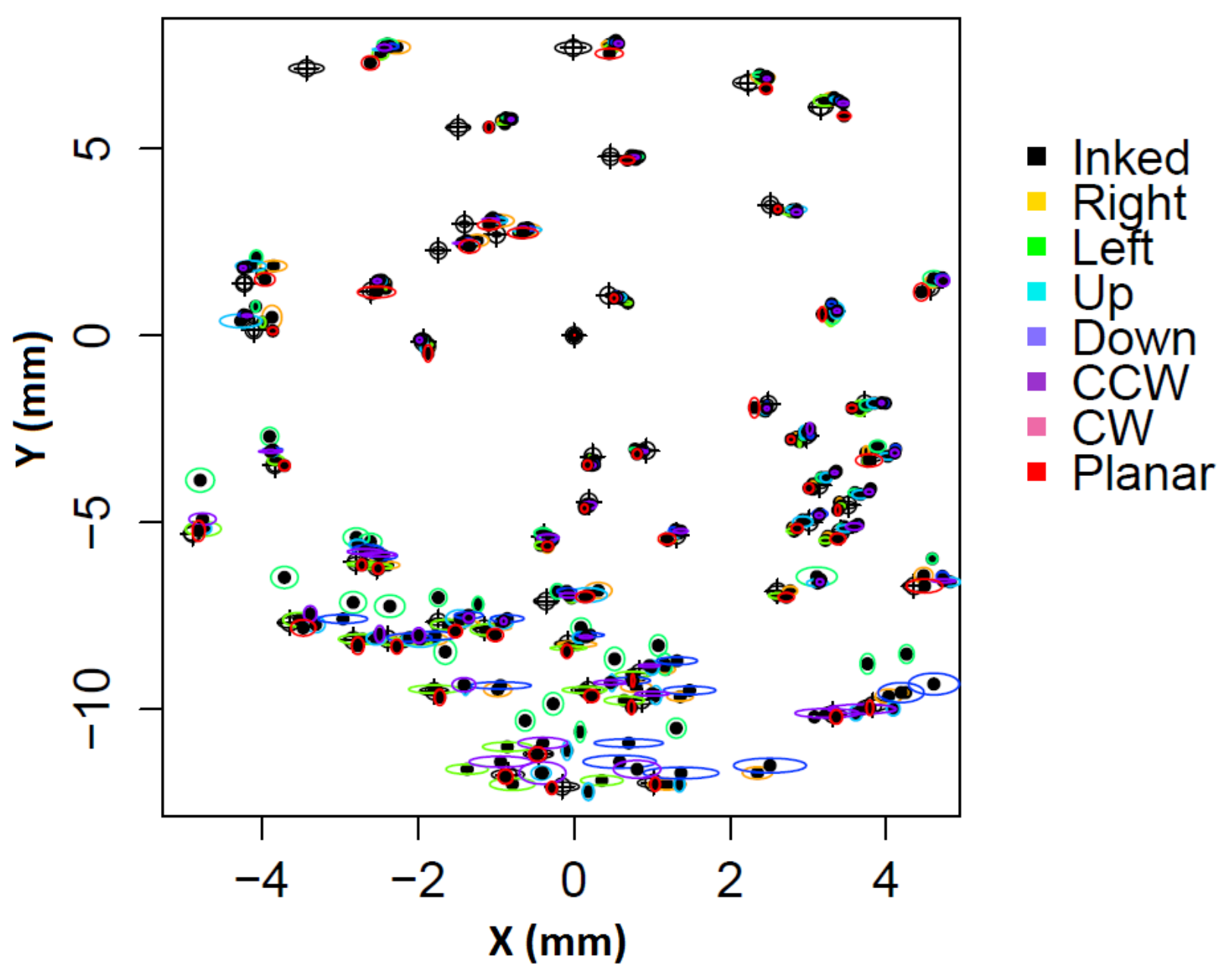


Finger 30: Plain Arch

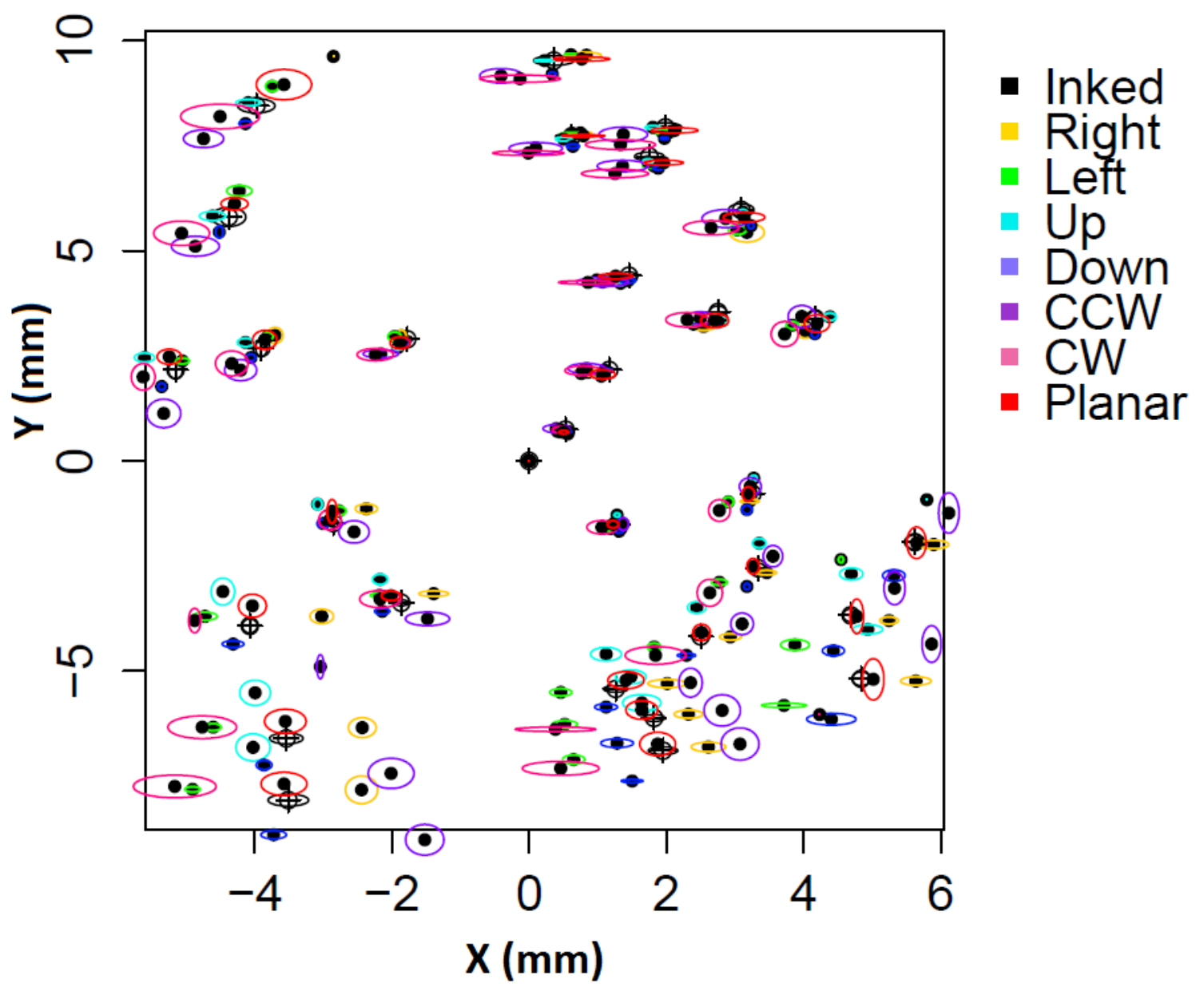


Appendix C (Normality Plots)
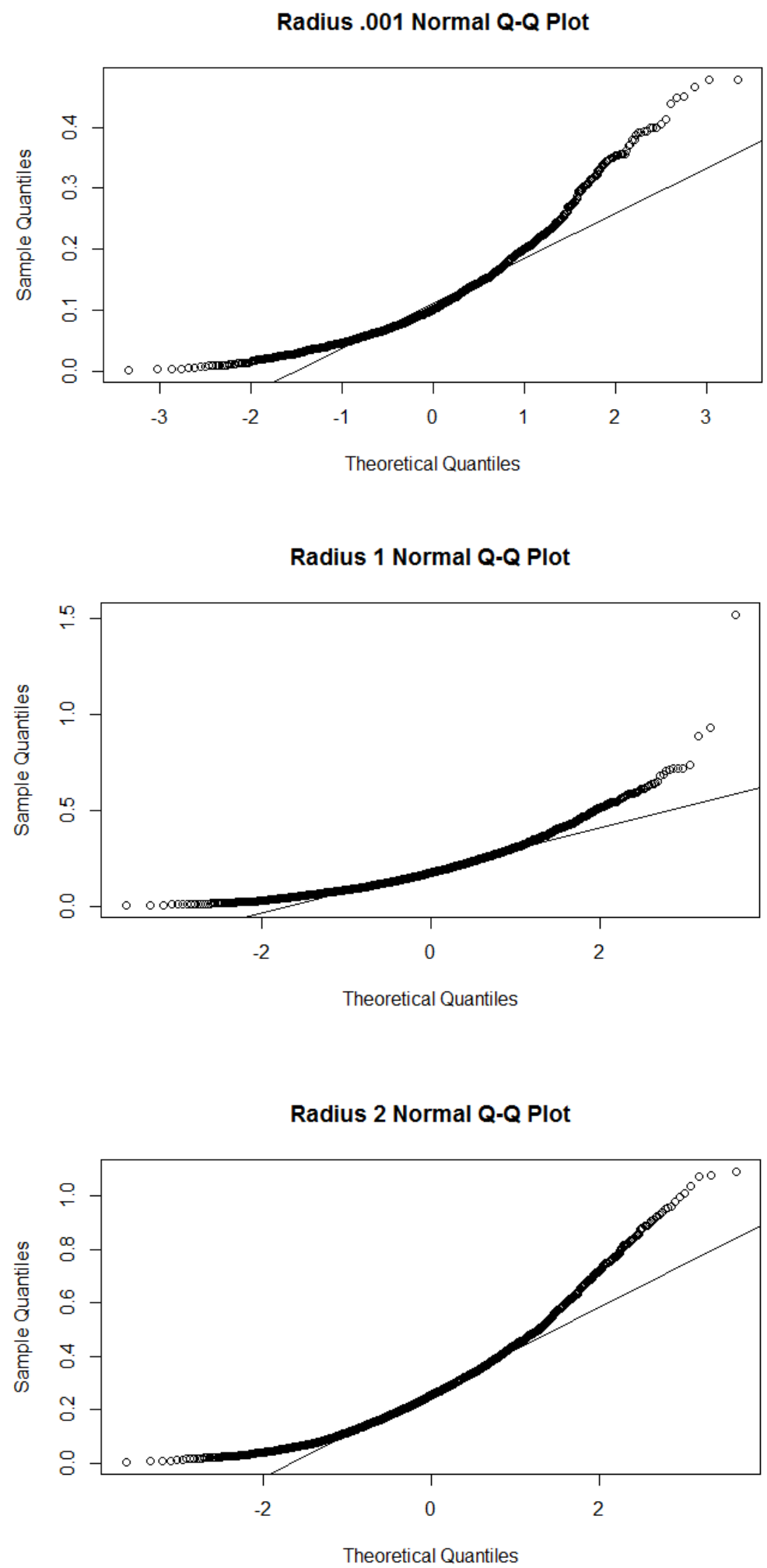

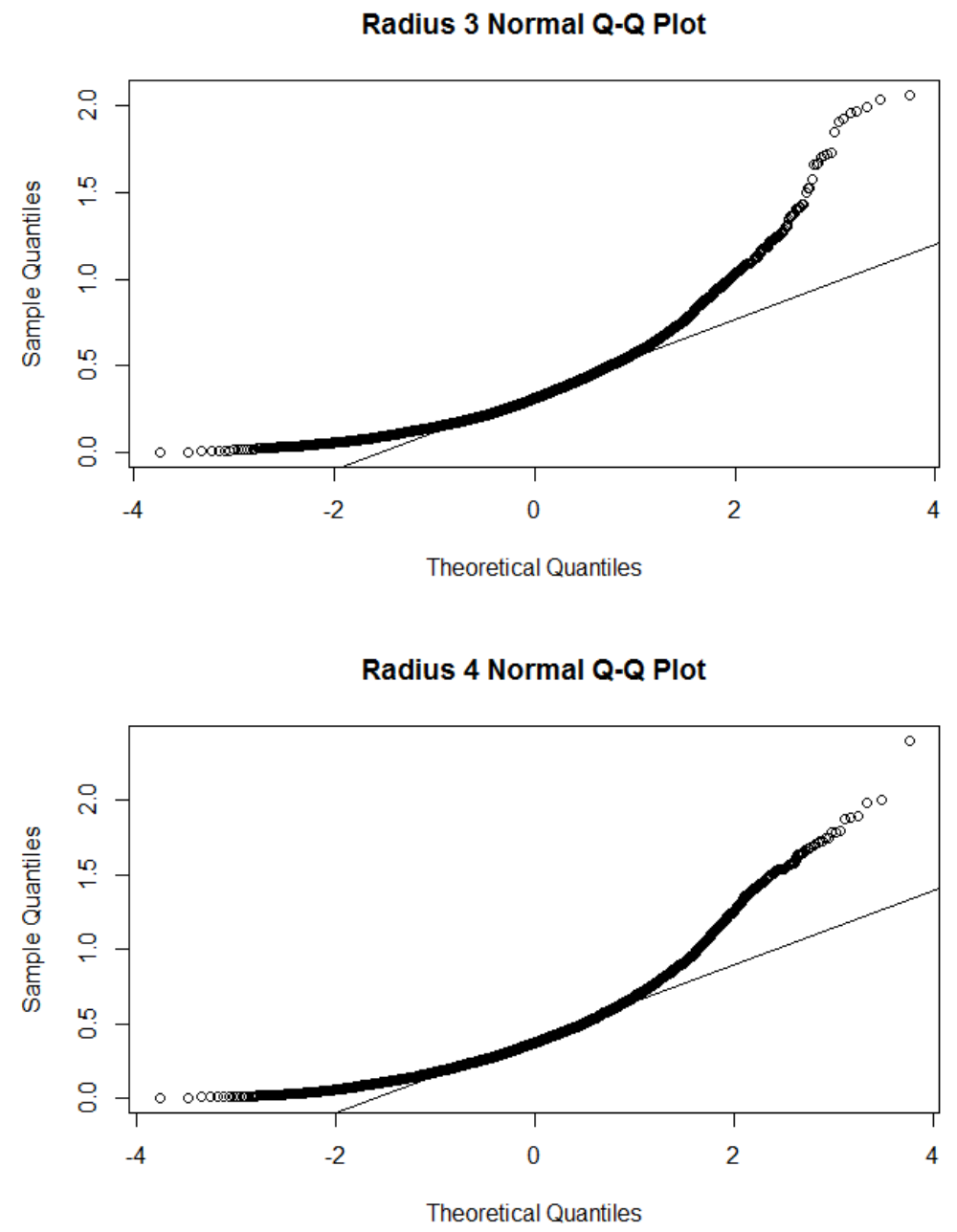

Radius 5 Normal Q-Q Plot

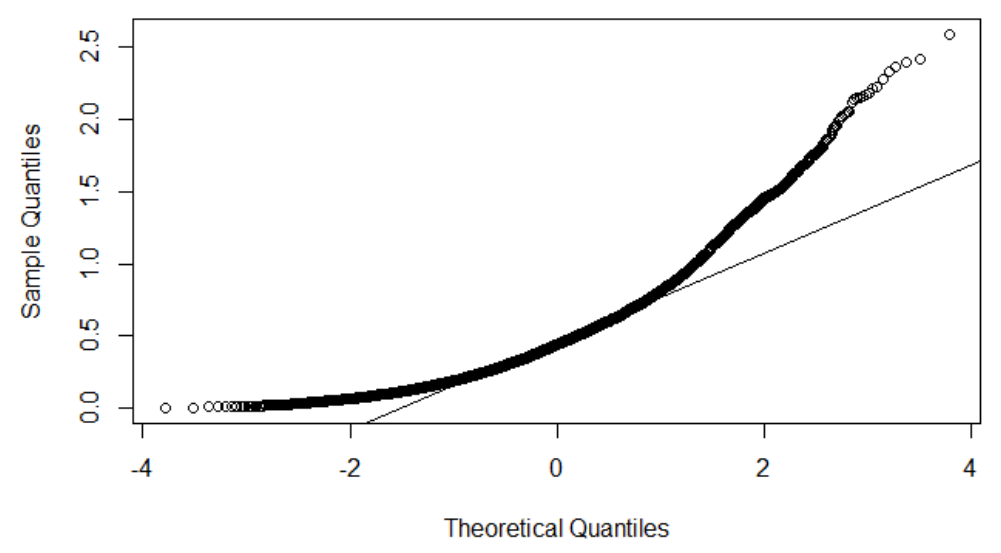


Radius 6 Normal Q-Q Plot

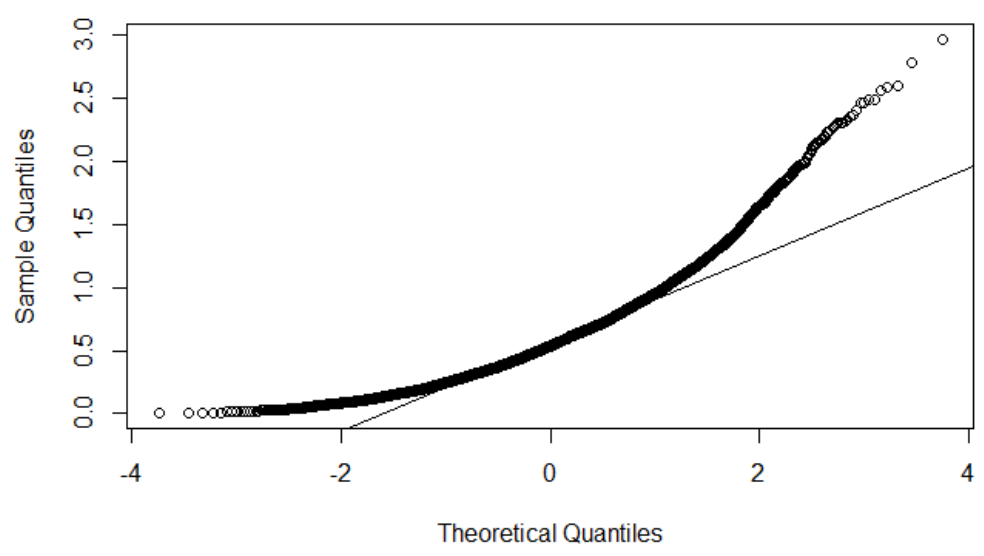

Radius 7 Normal Q-Q Plot

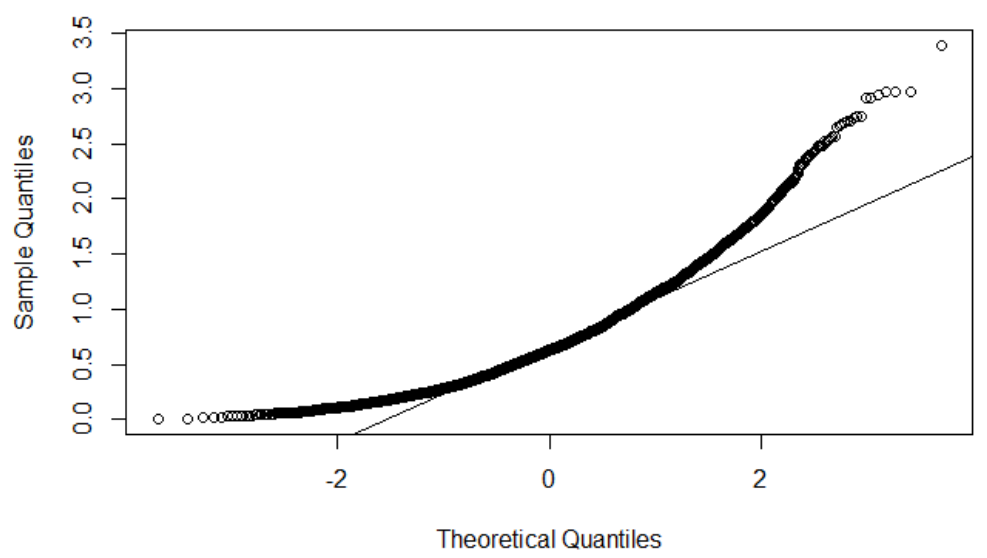

Radius 8 Normal Q-Q Plot

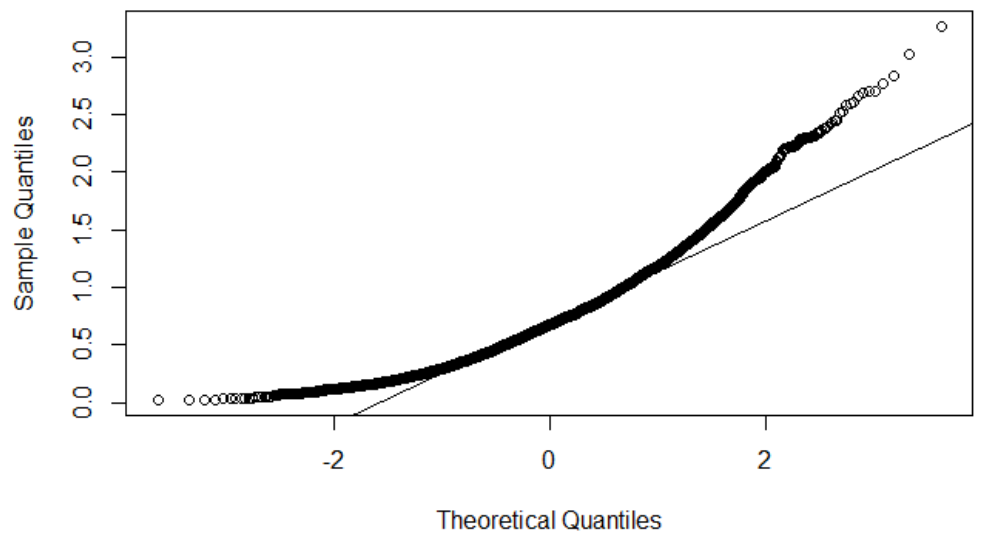



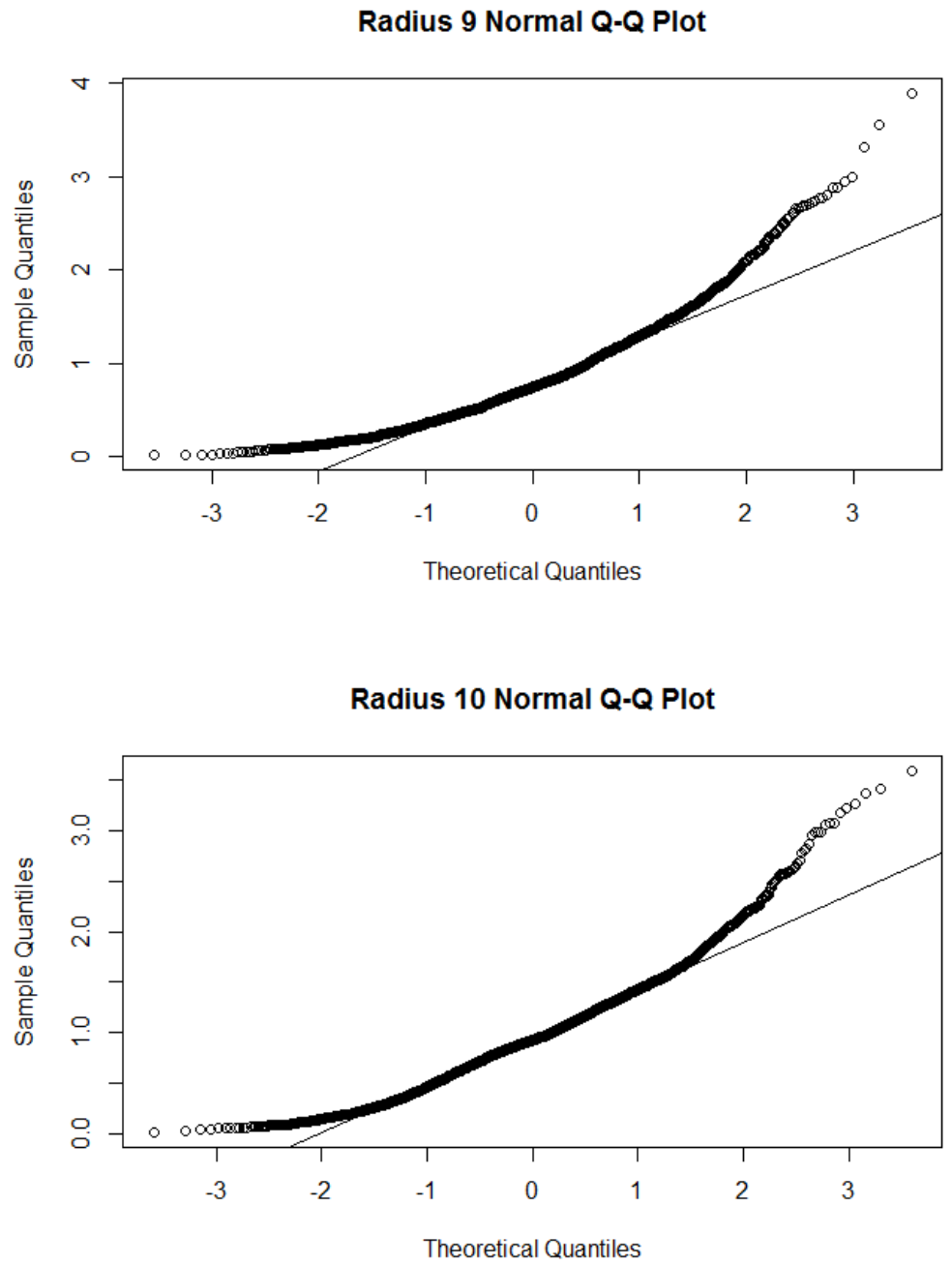

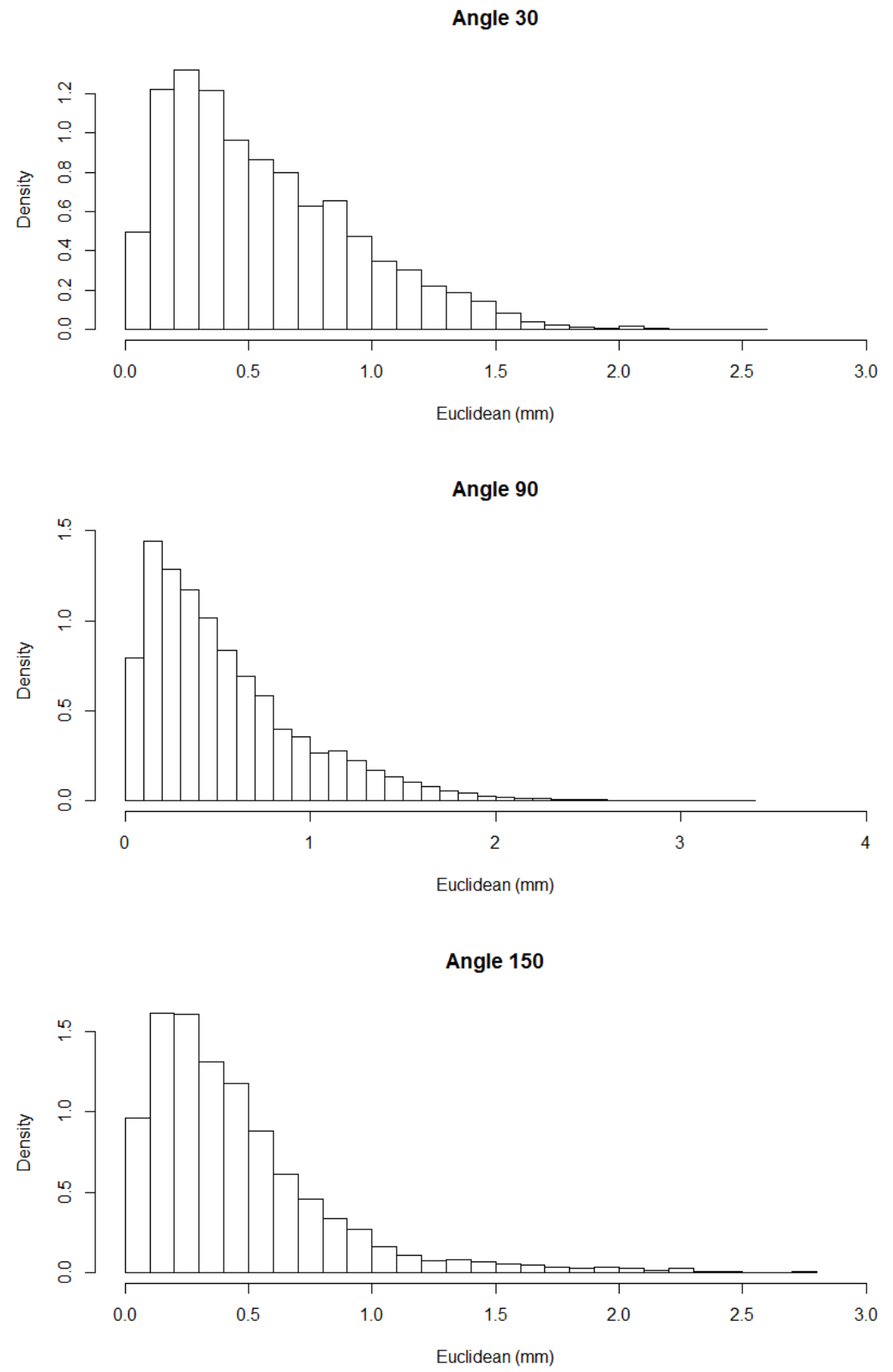

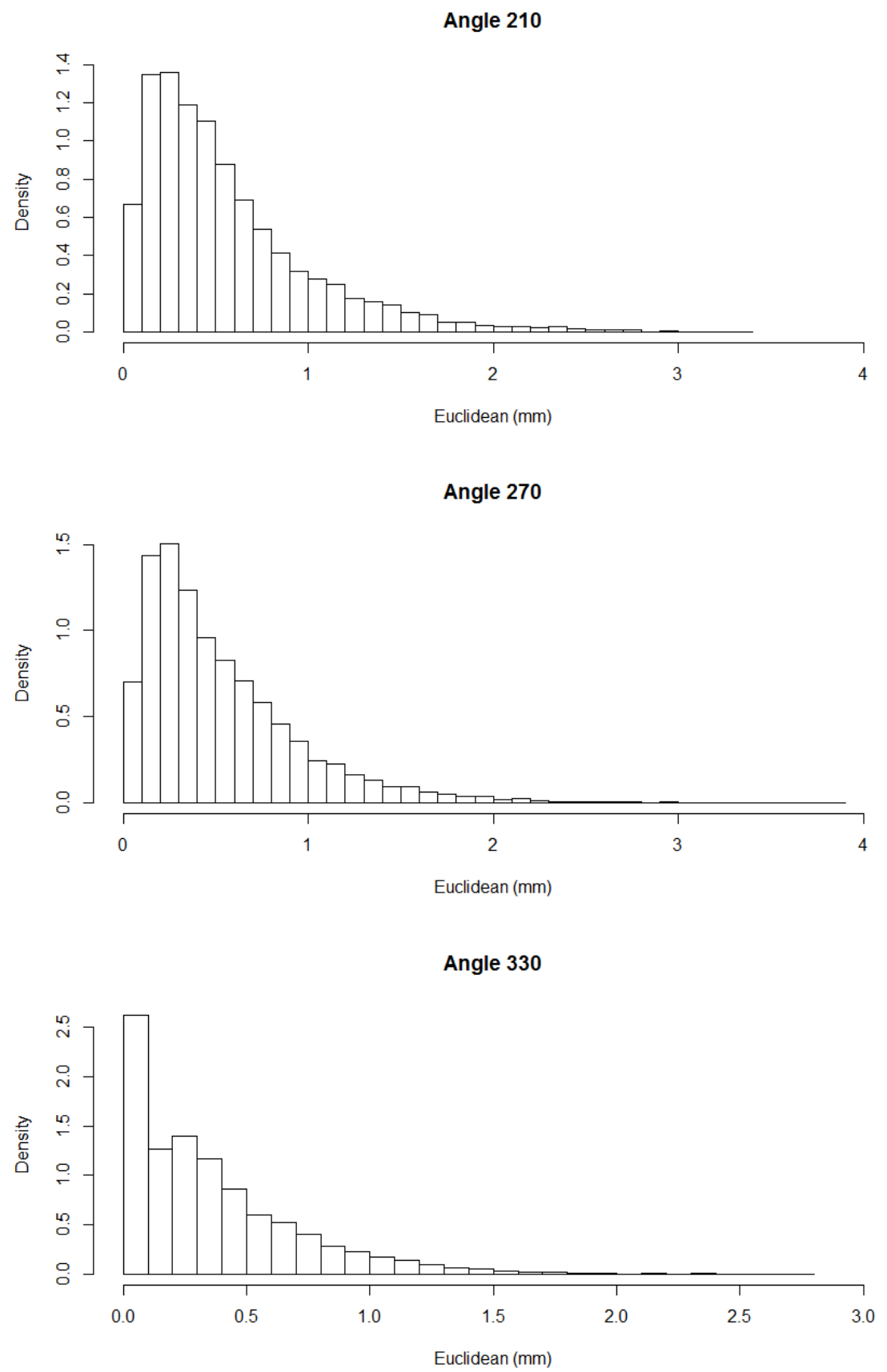


\section{Frequency of Minutiae Displacements}

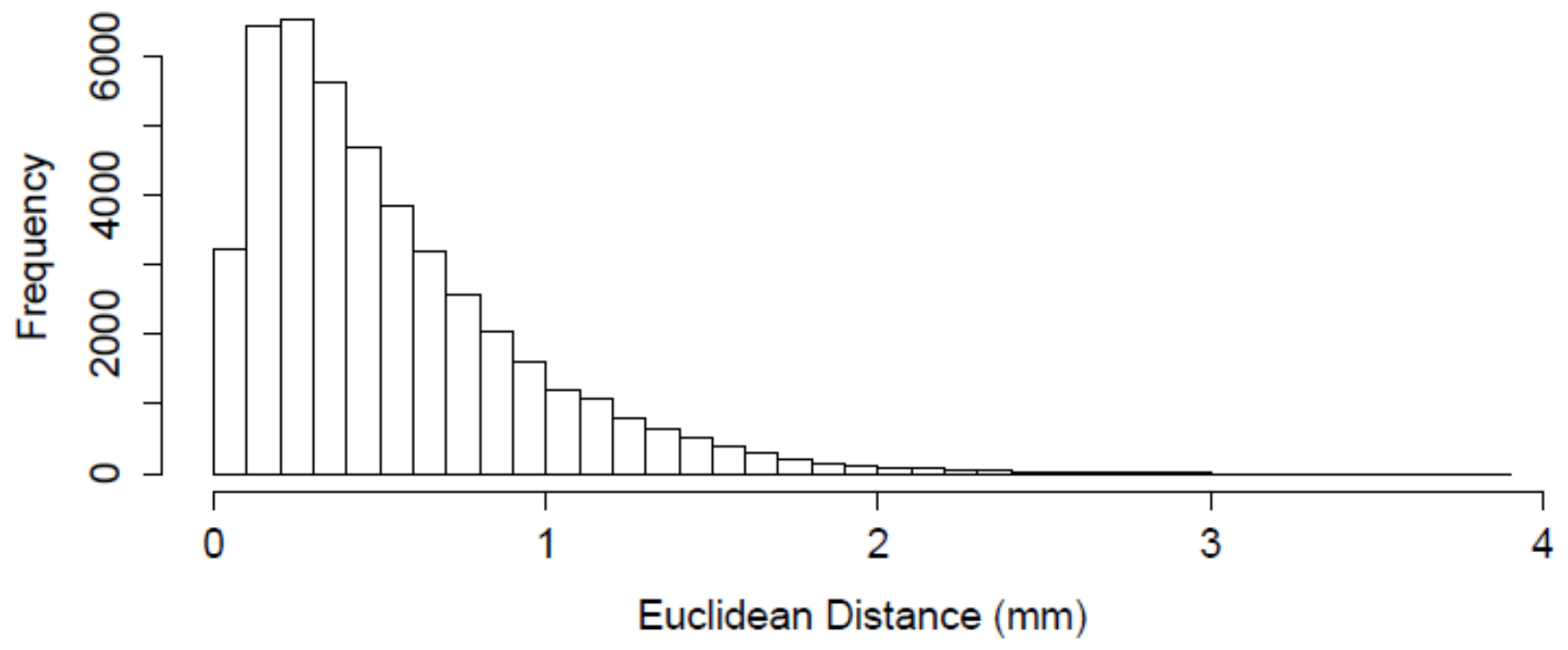


Appendix D (ANOVA and Tukey Test Results)

ANOVA and Tukey Tests for Determination of Radial and Angular Bins

ANOVA Summary of Radius Data

\begin{tabular}{lccclc}
\hline & Df & Sum Sq & Mean Sq & F value & $\operatorname{Pr}(>\mathrm{F})$ \\
\hline radiusANOVA\$Radius & 10 & 2162 & 216.23 & 1693 & $<2 \mathrm{e}-16$ \\
Residuals & 45476 & 5808 & 0.13 & & \\
\hline
\end{tabular}

Tukey Comparison of Radial Bin Euclidean Means

\begin{tabular}{|c|c|c|c|c|}
\hline Radius Comparison & diff & Iwr & upr & $p$ adj \\
\hline Radius 10-Radius 1 & 0.703264 & $6.63 \mathrm{E}-01$ & $7.43 \mathrm{E}-01$ & $0.00 E+00$ \\
\hline Radius 11-Radius 1 & 0.845267 & 8.06E-01 & $8.84 \mathrm{E}-01$ & $0.00 E+00$ \\
\hline Radius 2-Radius 1 & 0.07526 & $3.66 \mathrm{E}-02$ & 1.14E-01 & $2.08 \mathrm{E}-08$ \\
\hline Radius 3-Radius 1 & 0.158931 & $1.21 \mathrm{E}-01$ & $1.97 \mathrm{E}-01$ & $0.00 E+00$ \\
\hline Radius 4-Radius 1 & 0.242563 & 2.06E-01 & 2.79E-01 & $0.00 E+00$ \\
\hline Radius 5-Radius 1 & 0.313871 & $2.78 \mathrm{E}-01$ & $3.50 \mathrm{E}-01$ & $0.00 \mathrm{E}+00$ \\
\hline Radius 6-Radius 1 & 0.389427 & $3.54 \mathrm{E}-01$ & 4.25E-01 & $0.00 E+00$ \\
\hline Radius 7-Radius 1 & 0.485782 & 4.49E-01 & $5.22 \mathrm{E}-01$ & $0.00 E+00$ \\
\hline Radius 8-Radius 1 & 0.59057 & $5.54 \mathrm{E}-01$ & $6.28 \mathrm{E}-01$ & $0.00 E+00$ \\
\hline Radius 9-Radius 1 & 0.628859 & 5.91E-01 & 6.67E-01 & $0.00 \mathrm{E}+00$ \\
\hline Radius 11 -Radius 10 & 0.142003 & $1.11 \mathrm{E}-01$ & $1.73 \mathrm{E}-01$ & $0.00 E+00$ \\
\hline Radius 2-Radius 10 & -0.628 & $-6.58 \mathrm{E}-01$ & $-5.98 \mathrm{E}-01$ & $0.00 E+00$ \\
\hline Radius 3-Radius 10 & -0.54433 & $-5.74 \mathrm{E}-01$ & $-5.15 E-01$ & $0.00 E+00$ \\
\hline Radius 4-Radius 10 & -0.4607 & $-4.88 \mathrm{E}-01$ & $-4.33 E-01$ & $0.00 E+00$ \\
\hline Radius 5-Radius 10 & -0.38939 & $-4.16 \mathrm{E}-01$ & $-3.62 E-01$ & $0.00 \mathrm{E}+00$ \\
\hline Radius 6-Radius 10 & -0.31384 & $-3.41 \mathrm{E}-01$ & $-2.87 \mathrm{E}-01$ & $0.00 \mathrm{E}+00$ \\
\hline Radius 7-Radius 10 & -0.21748 & $-2.45 \mathrm{E}-01$ & $-1.90 \mathrm{E}-01$ & $0.00 \mathrm{E}+00$ \\
\hline Radius 8-Radius 10 & -0.11269 & $-1.41 \mathrm{E}-01$ & $-8.44 \mathrm{E}-02$ & $0.00 E+00$ \\
\hline Radius 9-Radius 10 & -0.0744 & $-1.04 \mathrm{E}-01$ & $-4.46 \mathrm{E}-02$ & $1.98 \mathrm{E}-13$ \\
\hline Radius 2-Radius 11 & -0.77001 & $-7.99 \mathrm{E}-01$ & $-7.41 E-01$ & $0.00 E+00$ \\
\hline Radius 3-Radius 11 & -0.68634 & $-7.15 \mathrm{E}-01$ & $-6.58 \mathrm{E}-01$ & $0.00 E+00$ \\
\hline Radius 4-Radius 11 & -0.6027 & $-6.29 E-01$ & $-5.77 E-01$ & $0.00 E+00$ \\
\hline Radius 5-Radius 11 & -0.5314 & $-5.57 E-01$ & $-5.06 \mathrm{E}-01$ & $0.00 E+00$ \\
\hline Radius 6-Radius 11 & -0.45584 & $-4.81 \mathrm{E}-01$ & $-4.31 E-01$ & $0.00 E+00$ \\
\hline Radius 7-Radius 11 & -0.35948 & $-3.85 \mathrm{E}-01$ & $-3.34 \mathrm{E}-01$ & $0.00 E+00$ \\
\hline Radius 8-Radius 11 & -0.2547 & $-2.81 \mathrm{E}-01$ & $-2.28 \mathrm{E}-01$ & $0.00 \mathrm{E}+00$ \\
\hline Radius 9-Radius 11 & -0.21641 & $-2.45 \mathrm{E}-01$ & $-1.88 \mathrm{E}-01$ & $0.00 \mathrm{E}+00$ \\
\hline Radius 3-Radius 2 & 0.083671 & 5.57E-02 & $1.12 \mathrm{E}-01$ & 7.93E-14 \\
\hline Radius 4-Radius 2 & 0.167304 & $1.42 \mathrm{E}-01$ & $1.93 \mathrm{E}-01$ & $0.00 E+00$ \\
\hline Radius 5-Radius 2 & 0.238611 & $2.14 \mathrm{E}-01$ & $2.64 \mathrm{E}-01$ & $0.00 E+00$ \\
\hline Radius 6-Radius 2 & 0.314167 & $2.89 \mathrm{E}-01$ & 3.39E-01 & $0.00 \mathrm{E}+00$ \\
\hline Radius 7-Radius 2 & 0.410522 & $3.85 \mathrm{E}-01$ & 4.36E-01 & $0.00 \mathrm{E}+00$ \\
\hline Radius 8-Radius 2 & 0.51531 & $4.89 \mathrm{E}-01$ & $5.42 \mathrm{E}-01$ & $0.00 E+00$ \\
\hline Radius 9-Radius 2 & 0.553599 & $5.26 \mathrm{E}-01$ & $5.82 \mathrm{E}-01$ & $0.00 E+00$ \\
\hline Radius 4-Radius 3 & 0.083633 & $5.89 \mathrm{E}-02$ & $1.08 \mathrm{E}-01$ & $1.03 \mathrm{E}-13$ \\
\hline Radius 5-Radius 3 & 0.15494 & $1.31 \mathrm{E}-01$ & $1.79 \mathrm{E}-01$ & $0.00 E+00$ \\
\hline Radius 6-Radius 3 & 0.230496 & 2.07E-01 & $2.54 \mathrm{E}-01$ & $0.00 \mathrm{E}+00$ \\
\hline Radius 7-Radius 3 & 0.326851 & $3.02 \mathrm{E}-01$ & $3.52 \mathrm{E}-01$ & $0.00 E+00$ \\
\hline Radius 8-Radius 3 & 0.431639 & $4.06 \mathrm{E}-01$ & 4.57E-01 & $0.00 E+00$ \\
\hline Radius 9-Radius 3 & 0.469928 & $4.43 \mathrm{E}-01$ & 4.97E-01 & $0.00 E+00$ \\
\hline Radius 5-Radius 4 & 0.071307 & 4.99E-02 & $9.28 \mathrm{E}-02$ & $1.03 \mathrm{E}-13$ \\
\hline Radius 6-Radius 4 & 0.146863 & $1.26 \mathrm{E}-01$ & $1.68 \mathrm{E}-01$ & $0.00 E+00$ \\
\hline
\end{tabular}




\begin{tabular}{lllll} 
Radius 7-Radius 4 & 0.243218 & $2.21 \mathrm{E}-01$ & $2.65 \mathrm{E}-01$ & $0.00 \mathrm{E}+00$ \\
Radius 8-Radius 4 & 0.348006 & $3.25 \mathrm{E}-01$ & $3.71 \mathrm{E}-01$ & $0.00 \mathrm{E}+00$ \\
Radius 9-Radius 4 & 0.386296 & $3.61 \mathrm{E}-01$ & $4.11 \mathrm{E}-01$ & $0.00 \mathrm{E}+00$ \\
Radius 6-Radius 5 & 0.075556 & $5.51 \mathrm{E}-02$ & $9.60 \mathrm{E}-02$ & $0.00 \mathrm{E}+00$ \\
Radius 7-Radius 5 & 0.171911 & $1.50 \mathrm{E}-01$ & $1.93 \mathrm{E}-01$ & $0.00 \mathrm{E}+00$ \\
Radius 8-Radius 5 & 0.276699 & $2.54 \mathrm{E}-01$ & $2.99 \mathrm{E}-01$ & $0.00 \mathrm{E}+00$ \\
Radius 9-Radius 5 & 0.314988 & $2.91 \mathrm{E}-01$ & $3.39 \mathrm{E}-01$ & $0.00 \mathrm{E}+00$ \\
Radius 7-Radius 6 & 0.096355 & $7.54 \mathrm{E}-02$ & $1.17 \mathrm{E}-01$ & $0.00 \mathrm{E}+00$ \\
Radius 8-Radius 6 & 0.201143 & $1.79 \mathrm{E}-01$ & $2.23 \mathrm{E}-01$ & $0.00 \mathrm{E}+00$ \\
Radius 9-Radius 6 & 0.239433 & $2.15 \mathrm{E}-01$ & $2.63 \mathrm{E}-01$ & $0.00 \mathrm{E}+00$ \\
Radius 8-Radius 7 & 0.104788 & $8.19 \mathrm{E}-02$ & $1.28 \mathrm{E}-01$ & $0.00 \mathrm{E}+00$ \\
Radius 9-Radius 7 & 0.143078 & $1.18 \mathrm{E}-01$ & $1.68 \mathrm{E}-01$ & $0.00 \mathrm{E}+00$ \\
Radius 9-Radius 8 & 0.038289 & $1.26 \mathrm{E}-02$ & $6.40 \mathrm{E}-02$ & $8.89 \mathrm{E}-05$ \\
\hline
\end{tabular}

ANOVA Summary of 30 Angle Data

\begin{tabular}{lccclc}
\hline & Df & Sum Sq & Mean Sq & $F$ value & $\operatorname{Pr}(>F)$ \\
\hline Angle\$Angle & 11 & 128 & 11.660 & 67.61 & $<2 \mathrm{e}-16$ \\
Residua1s & 45475 & 7842 & 0.172 & & \\
\hline
\end{tabular}

Tukey Comparison of $30^{\circ}$ Angle Bin Euclidean Means

\begin{tabular}{|c|c|c|c|c|}
\hline Angle Comparison & diff & Iwr & upr & $p$ adj \\
\hline Angle 120-Angle 0 & 0.017702 & $-2.37 \mathrm{E}-02$ & $5.91 \mathrm{E}-02$ & $9.64 \mathrm{E}-01$ \\
\hline Angle 150 -Angle 0 & -0.03855 & $-8.48 \mathrm{E}-02$ & $7.68 \mathrm{E}-03$ & $2.14 \mathrm{E}-01$ \\
\hline Angle 180-Angle 0 & 0.035966 & $-7.61 \mathrm{E}-03$ & $7.95 \mathrm{E}-02$ & $2.27 \mathrm{E}-01$ \\
\hline Angle 210-Angle 0 & 0.053368 & $1.35 \mathrm{E}-02$ & $9.32 \mathrm{E}-02$ & 7.39E-04 \\
\hline Angle 240-Angle 0 & 0.147202 & $1.10 \mathrm{E}-01$ & $1.85 \mathrm{E}-01$ & $0.00 \mathrm{E}+00$ \\
\hline Angle 270-Angle 0 & 0.097451 & $5.98 \mathrm{E}-02$ & $1.35 \mathrm{E}-01$ & $1.19 \mathrm{E}-13$ \\
\hline Angle 30 -Angle 0 & 0.049753 & $7.11 \mathrm{E}-03$ & $9.24 \mathrm{E}-02$ & 7.63E-03 \\
\hline Angle 300-Angle 0 & 0.050346 & $1.19 \mathrm{E}-02$ & 8.88E-02 & $1.16 \mathrm{E}-03$ \\
\hline Angle 330-Angle 0 & $1.73 \mathrm{E}-05$ & $-4.27 \mathrm{E}-02$ & 4.27E-02 & $1.00 \mathrm{E}+00$ \\
\hline Angle 60-Angle 0 & 0.132413 & $9.46 \mathrm{E}-02$ & $1.70 \mathrm{E}-01$ & $0.00 \mathrm{E}+00$ \\
\hline Angle 90 -Angle 0 & 0.118295 & $8.10 \mathrm{E}-02$ & $1.56 \mathrm{E}-01$ & $1.08 \mathrm{E}-13$ \\
\hline Angle 150-Angle 120 & -0.05625 & $-9.70 \mathrm{E}-02$ & $-1.55 \mathrm{E}-02$ & $3.98 \mathrm{E}-04$ \\
\hline Angle 180-Angle 120 & 0.018264 & $-1.94 \mathrm{E}-02$ & $5.60 \mathrm{E}-02$ & $9.15 \mathrm{E}-01$ \\
\hline Angle 210-Angle 120 & 0.035666 & $2.35 \mathrm{E}-03$ & $6.90 \mathrm{E}-02$ & $2.37 \mathrm{E}-02$ \\
\hline Angle 240-Angle 120 & 0.1295 & $9.88 \mathrm{E}-02$ & $1.60 \mathrm{E}-01$ & $0.00 \mathrm{E}+00$ \\
\hline Angle 270-Angle 120 & 0.079749 & 4.91E-02 & $1.10 \mathrm{E}-01$ & $1.14 \mathrm{E}-13$ \\
\hline Angle 30-Angle 120 & 0.032051 & $-4.56 \mathrm{E}-03$ & $6.87 \mathrm{E}-02$ & $1.55 \mathrm{E}-01$ \\
\hline Angle 300-Angle 120 & 0.032644 & $9.64 \mathrm{E}-04$ & $6.43 \mathrm{E}-02$ & $3.65 \mathrm{E}-02$ \\
\hline Angle 330-Angle 120 & -0.01768 & $-5.44 \mathrm{E}-02$ & $1.90 \mathrm{E}-02$ & $9.18 \mathrm{E}-01$ \\
\hline Angle 60-Angle 120 & 0.114711 & 8.39E-02 & $1.46 \mathrm{E}-01$ & $0.00 \mathrm{E}+00$ \\
\hline Angle 90-Angle 120 & 0.100593 & 7.04E-02 & $1.31 \mathrm{E}-01$ & $1.08 \mathrm{E}-13$ \\
\hline Angle 180-Angle 150 & 0.074513 & $3.16 \mathrm{E}-02$ & $1.17 \mathrm{E}-01$ & $9.37 \mathrm{E}-07$ \\
\hline Angle 210-Angle 150 & 0.091915 & $5.27 \mathrm{E}-02$ & $1.31 \mathrm{E}-01$ & $1.22 \mathrm{E}-12$ \\
\hline Angle 240-Angle 150 & 0.185749 & $1.49 \mathrm{E}-01$ & $2.23 \mathrm{E}-01$ & $0.00 \mathrm{E}+00$ \\
\hline Angle 270-Angle 150 & 0.135998 & $9.91 \mathrm{E}-02$ & $1.73 \mathrm{E}-01$ & $0.00 \mathrm{E}+00$ \\
\hline Angle 30 -Angle 150 & 0.0883 & 4.63E-02 & $1.30 \mathrm{E}-01$ & $4.25 \mathrm{E}-10$ \\
\hline Angle 300-Angle 150 & 0.088893 & $5.11 \mathrm{E}-02$ & $1.27 \mathrm{E}-01$ & $1.06 \mathrm{E}-12$ \\
\hline Angle 330-Angle 150 & 0.038565 & $-3.50 \mathrm{E}-03$ & $8.06 \mathrm{E}-02$ & $1.09 \mathrm{E}-01$ \\
\hline Angle 60 -Angle 150 & 0.17096 & $1.34 \mathrm{E}-01$ & $2.08 \mathrm{E}-01$ & $0.00 \mathrm{E}+00$ \\
\hline Angle 90 -Angle 150 & 0.156842 & $1.20 \mathrm{E}-01$ & $1.93 \mathrm{E}-01$ & $0.00 \mathrm{E}+00$ \\
\hline Angle 210-Angle 180 & 0.017402 & $-1.86 \mathrm{E}-02$ & 5.34E-02 & $9.17 \mathrm{E}-01$ \\
\hline Angle 240-Angle 180 & 0.111236 & $7.77 \mathrm{E}-02$ & $1.45 \mathrm{E}-01$ & $1.16 \mathrm{E}-13$ \\
\hline Angle 270-Angle 180 & 0.061485 & $2.80 \mathrm{E}-02$ & $9.50 \mathrm{E}-02$ & $1.35 \mathrm{E}-07$ \\
\hline Angle 30-Angle 180 & 0.013787 & $-2.53 \mathrm{E}-02$ & $5.28 \mathrm{E}-02$ & $9.92 \mathrm{E}-01$ \\
\hline Angle 300-Angle 180 & 0.01438 & $-2.01 \mathrm{E}-02$ & 4.89E-02 & $9.70 \mathrm{E}-01$ \\
\hline Angle 330-Angle 180 & -0.03595 & $-7.51 \mathrm{E}-02$ & $3.17 \mathrm{E}-03$ & $1.07 \mathrm{E}-01$ \\
\hline Angle 60-Angle 180 & 0.096447 & $6.27 \mathrm{E}-02$ & $1.30 \mathrm{E}-01$ & $1.19 \mathrm{E}-13$ \\
\hline
\end{tabular}


Angle 90-Angle 180

Angle 240-Angle 210

Angle 270-Angle 210

Angle 30-Angle 210

Angle 300-Angle 210

Angle 330-Angle 210

Angle 60-Angle 210

Angle 90-Angle 210

Angle 270-Angle 240

Angle 30-Angle 240

Angle 300-Angle 240

Angle 330-Angle 240

Angle 60-Angle 240

Angle 90-Angle 240

Angle 30-Angle 270

Angle 300-Angle 270

Angle 330-Angle 270

Angle 60-Angle 270

Angle 90-Angle 270

Angle 300-Angle 30

Angle 330-Angle 30

Angle 60-Angle 30

Angle 90-Angle 30

Angle 330-Angle 300

Angle 60-Angle 300

Angle 90-Angle 300

Angle 60-Angle 330

Angle 90-Angle 330

Angle 90-Angle 60

\begin{tabular}{llll}
0.082329 & $4.92 \mathrm{E}-02$ & $1.15 \mathrm{E}-01$ & $1.78 \mathrm{E}-13$ \\
0.093834 & $6.53 \mathrm{E}-02$ & $1.22 \mathrm{E}-01$ & $1.19 \mathrm{E}-13$ \\
0.044083 & $1.56 \mathrm{E}-02$ & $7.26 \mathrm{E}-02$ & $2.84 \mathrm{E}-05$ \\
-0.00361 & $-3.85 \mathrm{E}-02$ & $3.12 \mathrm{E}-02$ & $1.00 \mathrm{E}+00$ \\
-0.00302 & $-3.27 \mathrm{E}-02$ & $2.66 \mathrm{E}-02$ & $1.00 \mathrm{E}+00$ \\
-0.05335 & $-8.83 \mathrm{E}-02$ & $-1.84 \mathrm{E}-02$ & $3.85 \mathrm{E}-05$ \\
0.079045 & $5.03 \mathrm{E}-02$ & $1.08 \mathrm{E}-01$ & $8.17 \mathrm{E}-14$ \\
0.064927 & $3.69 \mathrm{E}-02$ & $9.30 \mathrm{E}-02$ & $2.73 \mathrm{E}-12$ \\
-0.04975 & $-7.52 \mathrm{E}-02$ & $-2.43 \mathrm{E}-02$ & $1.03 \mathrm{E}-08$ \\
-0.09745 & $-1.30 \mathrm{E}-01$ & $-6.51 \mathrm{E}-02$ & $1.18 \mathrm{E}-13$ \\
-0.09686 & $-1.24 \mathrm{E}-01$ & $-7.02 \mathrm{E}-02$ & $0.00 \mathrm{E}+00$ \\
-0.14718 & $-1.80 \mathrm{E}-01$ & $-1.15 \mathrm{E}-01$ & $0.00 \mathrm{E}+00$ \\
-0.01479 & $-4.04 \mathrm{E}-02$ & $1.09 \mathrm{E}-02$ & $7.70 \mathrm{E}-01$ \\
-0.02891 & $-5.38 \mathrm{E}-02$ & $-4.02 \mathrm{E}-03$ & $8.14 \mathrm{E}-03$ \\
-0.0477 & $-8.00 \mathrm{E}-02$ & $-1.54 \mathrm{E}-02$ & $8.94 \mathrm{E}-05$ \\
-0.04711 & $-7.37 \mathrm{E}-02$ & $-2.05 \mathrm{E}-02$ & $4.64 \mathrm{E}-07$ \\
-0.09743 & $-1.30 \mathrm{E}-01$ & $-6.51 \mathrm{E}-02$ & $1.18 \mathrm{E}-13$ \\
0.034962 & $9.37 \mathrm{E}-03$ & $6.06 \mathrm{E}-02$ & $4.97 \mathrm{E}-04$ \\
0.020844 & $-3.98 \mathrm{E}-03$ & $4.57 \mathrm{E}-02$ & $2.05 \mathrm{E}-01$ \\
0.000593 & $-3.27 \mathrm{E}-02$ & $3.39 \mathrm{E}-02$ & $1.00 \mathrm{E}+00$ \\
-0.04974 & $-8.78 \mathrm{E}-02$ & $-1.17 \mathrm{E}-02$ & $1.19 \mathrm{E}-03$ \\
0.08266 & $5.02 \mathrm{E}-02$ & $1.15 \mathrm{E}-01$ & $1.36 \mathrm{E}-13$ \\
0.068542 & $3.66 \mathrm{E}-02$ & $1.00 \mathrm{E}-01$ & $1.46 \mathrm{E}-10$ \\
-0.05033 & $-8.37 \mathrm{E}-02$ & $-1.70 \mathrm{E}-02$ & $5.29 \mathrm{E}-05$ \\
0.082067 & $5.52 \mathrm{E}-02$ & $1.09 \mathrm{E}-01$ & $9.46 \mathrm{E}-14$ \\
0.067949 & $4.19 \mathrm{E}-02$ & $9.40 \mathrm{E}-02$ & $1.13 \mathrm{E}-13$ \\
0.132396 & $9.98 \mathrm{E}-02$ & $1.65 \mathrm{E}-01$ & $0.00 \mathrm{E}+00$ \\
0.118278 & $8.63 \mathrm{E}-02$ & $1.50 \mathrm{E}-01$ & $0.00 \mathrm{E}+00$ \\
-0.01412 & $-3.92 \mathrm{E}-02$ & $1.10 \mathrm{E}-02$ & $7.96 \mathrm{E}-01$ \\
\hline & & &
\end{tabular}

ANOVA Summary of 60 Degree Angle Bins

\begin{tabular}{lccccc}
\hline & Df & Sum Sq & Mean Sq & $F$ value & $\operatorname{Pr}(>F)$ \\
\hline Angle\$Angle & 5 & 110 & 21.994 & 127.3 & $<2 \mathrm{e}-16$ \\
Residuals & 45481 & 7861 & 0.173 & & \\
\hline
\end{tabular}

Tukey Comparison of 60 Degree Angle Sections

\begin{tabular}{lcccl}
\hline Angle Comparison & diff & lwr & upr & p adj \\
\hline Angle 120-Angle 0 & -0.03321 & $-5.83 \mathrm{E}-02$ & $-8.16 \mathrm{E}-03$ & $2.18 \mathrm{E}-03$ \\
Angle 180-Angle 0 & 0.016774 & $-6.99 \mathrm{E}-03$ & $4.05 \mathrm{E}-02$ & $3.35 \mathrm{E}-01$ \\
Angle 240-Angle 0 & 0.092224 & $7.09 \mathrm{E}-02$ & $1.14 \mathrm{E}-01$ & $0.00 \mathrm{E}+00$ \\
Angle 300-Angle 0 & 0.002922 & $-2.00 \mathrm{E}-02$ & $2.58 \mathrm{E}-02$ & $9.99 \mathrm{E}-01$ \\
Angle 60-Angle 0 & 0.094942 & $7.37 \mathrm{E}-02$ & $1.16 \mathrm{E}-01$ & $0.00 \mathrm{E}+00$ \\
Angle 180-Angle 120 & 0.049986 & $2.70 \mathrm{E}-02$ & $7.30 \mathrm{E}-02$ & $8.55 \mathrm{E}-09$ \\
Angle 240-Angle 120 & 0.125435 & $1.05 \mathrm{E}-01$ & $1.46 \mathrm{E}-01$ & $0.00 \mathrm{E}+00$ \\
Angle 300-Angle 120 & 0.036134 & $1.41 \mathrm{E}-02$ & $5.82 \mathrm{E}-02$ & $4.58 \mathrm{E}-05$ \\
Angle 60-Angle 120 & 0.128153 & $1.08 \mathrm{E}-01$ & $1.49 \mathrm{E}-01$ & $0.00 \mathrm{E}+00$ \\
Angle 240-Angle 180 & 0.075449 & $5.66 \mathrm{E}-02$ & $9.43 \mathrm{E}-02$ & $0.00 \mathrm{E}+00$ \\
Angle 300-Angle 180 & -0.01385 & $-3.45 \mathrm{E}-02$ & $6.76 \mathrm{E}-03$ & $3.93 \mathrm{E}-01$ \\
Angle 60-Angle 180 & 0.078168 & $5.94 \mathrm{E}-02$ & $9.69 \mathrm{E}-02$ & $0.00 \mathrm{E}+00$
\end{tabular}




\begin{tabular}{lllll} 
Angle 300-Angle 240 & -0.0893 & $-1.07 \mathrm{E}-01$ & $-7.15 \mathrm{E}-02$ & $0.00 \mathrm{E}+00$ \\
Angle 60-Angle 240 & 0.002719 & $-1.28 \mathrm{E}-02$ & $1.83 \mathrm{E}-02$ & $9.96 \mathrm{E}-01$ \\
Angle 60-Angle 300 & 0.09202 & $7.44 \mathrm{E}-02$ & $1.10 \mathrm{E}-01$ & $0.00 \mathrm{E}+00$ \\
\hline
\end{tabular}

ANOVA Summary of 60 Degree Offset Angle Sections

\begin{tabular}{lrrccc}
\hline & Df & Sum Sq & Mean Sq & $F$ value & $\operatorname{Pr}(>F)$ \\
\hline Angle\$Angle & 5 & 65 & 12.951 & 74.5 & $<2 \mathrm{e}-16$ \\
Residua1s & 45481 & 7906 & 0.174 & & \\
\hline
\end{tabular}

Tukey Comparison of 60 Degree Offset Angle Bins

\begin{tabular}{lcllc}
\hline Angle Comparison & diff & lwr & upr & p adj \\
\hline Angle 120-Angle 0 & 0.085761 & $6.37 \mathrm{E}-02$ & $1.08 \mathrm{E}-01$ & $3.06 \mathrm{E}-14$ \\
Angle 180-Angle 0 & 0.003523 & $-2.26 \mathrm{E}-02$ & $2.96 \mathrm{E}-02$ & $9.99 \mathrm{E}-01$ \\
Angle 240-Angle 0 & 0.109935 & $8.79 \mathrm{E}-02$ & $1.32 \mathrm{E}-01$ & $0.00 \mathrm{E}+00$ \\
Angle 300-Angle 0 & 0.076049 & $5.44 \mathrm{E}-02$ & $9.77 \mathrm{E}-02$ & $4.73 \mathrm{E}-14$ \\
Angle 60-Angle 0 & 0.106284 & $8.37 \mathrm{E}-02$ & $1.29 \mathrm{E}-01$ & $0.00 \mathrm{E}+00$ \\
Angle 180-Angle 120 & -0.08224 & $-1.05 \mathrm{E}-01$ & $-5.99 \mathrm{E}-02$ & $5.56 \mathrm{E}-14$ \\
Angle 240-Angle 120 & 0.024174 & $6.77 \mathrm{E}-03$ & $4.16 \mathrm{E}-02$ & $1.07 \mathrm{E}-03$ \\
Angle 300-Angle 120 & -0.00971 & $-2.67 \mathrm{E}-02$ & $7.24 \mathrm{E}-03$ & $5.77 \mathrm{E}-01$ \\
Angle 60-Angle 120 & 0.020524 & $2.41 \mathrm{E}-03$ & $3.86 \mathrm{E}-02$ & $1.57 \mathrm{E}-02$ \\
Angle 240-Angle 180 & 0.106412 & $8.41 \mathrm{E}-02$ & $1.29 \mathrm{E}-01$ & $0.00 \mathrm{E}+00$ \\
Angle 300-Angle 180 & 0.072525 & $5.06 \mathrm{E}-02$ & $9.45 \mathrm{E}-02$ & $5.56 \mathrm{E}-14$ \\
Angle 60-Angle 180 & 0.102761 & $7.99 \mathrm{E}-02$ & $1.26 \mathrm{E}-01$ & $0.00 \mathrm{E}+00$ \\
Angle 300-Angle 240 & -0.03389 & $-5.07 \mathrm{E}-02$ & $-1.70 \mathrm{E}-02$ & $1.52 \mathrm{E}-07$ \\
Angle 60-Angle 240 & -0.00365 & $-2.17 \mathrm{E}-02$ & $1.44 \mathrm{E}-02$ & $9.93 \mathrm{E}-01$ \\
Angle 60-Angle 300 & 0.030236 & $1.26 \mathrm{E}-02$ & $4.78 \mathrm{E}-02$ & $1.43 \mathrm{E}-05$ \\
\hline
\end{tabular}

Tukey Test for Comparison of Pattern Type by Radial Bins

\begin{tabular}{lrrccc}
\hline \multicolumn{7}{c}{ ANOVA Summary of Pattern Radial Sections } \\
\hline & Df & Sum Sq & Mean Sq & F value & Pr $(>F)$ \\
\hline Pattern\$Pattern: Pattern\$Radius & 20 & 65 & 3.27 & 26.24 & $<2$ e-16 \\
Residuals & 45454 & 5673 & 0.12 & & \\
\hline
\end{tabular}

Tukey Test of Pattern Type Radial Bins

\begin{tabular}{lllll}
\hline Pattern:Radius Comparison & diff & Iwr & upr & p adj \\
\hline Right Slant:Radius 001-Plain Arch:Radius 001 & -0.05301 & $-1.53 \mathrm{E}-01$ & $4.66 \mathrm{E}-02$ & 0.984925 \\
Right Slant:Radius 001-Plain Whorl:Radius 001 & -0.03126 & $-1.19 \mathrm{E}-01$ & $5.67 \mathrm{E}-02$ & 0.999993 \\
Right Slant:Radius 1-Plain Arch:Radius 1 & -0.00875 & $-6.85 \mathrm{E}-02$ & $5.10 \mathrm{E}-02$ & 1 \\
Right Slant:Radius 1-Plain Whorl:Radius 1 & -0.03105 & $-8.78 \mathrm{E}-02$ & $2.57 \mathrm{E}-02$ & 0.977789 \\
Right Slant:Radius 2-Plain Arch:Radius 2 & -0.02663 & $-8.54 \mathrm{E}-02$ & $3.21 \mathrm{E}-02$ & 0.998911 \\
Right Slant:Radius 2-Plain Whorl:Radius 2 & -0.03702 & $-8.83 \mathrm{E}-02$ & $1.42 \mathrm{E}-02$ & 0.641976 \\
Right Slant:Radius 3-Plain Arch:Radius 3 & 0.026332 & $-1.93 \mathrm{E}-02$ & $7.19 \mathrm{E}-02$ & 0.955108 \\
Right Slant:Radius 3-Plain Whorl:Radius 3 & -0.0142 & $-5.75 \mathrm{E}-02$ & $2.91 \mathrm{E}-02$ & 0.999999
\end{tabular}


Right Slant:Radius 6-Plain Whorl:Radius 6

Right Slant:Radius 10-Plain Arch:Radius 10

Right Slant:Radius 10-Plain Whorl:Radius 10

Right Slant:Radius 4-Plain Arch:Radius 4

Right Slant:Radius 4-Plain Whorl:Radius 4

Right Slant:Radius 5-Plain Arch:Radius 5

Right Slant:Radius 5-Plain Whorl:Radius 5

Right Slant:Radius 6-Plain Arch:Radius 6

Right Slant:Radius 7-Plain Arch:Radius 7

Right Slant:Radius 7-Plain Whorl:Radius 7

Right Slant:Radius 8-Plain Arch:Radius 8

Right Slant:Radius 8-Plain Whorl:Radius 8

Right Slant:Radius 9-Plain Arch:Radius 9

Right Slant:Radius 9-Plain Whorl:Radius 9

*** green highlighted cell indicated

distinguishable

$\begin{array}{llll}-0.02146 & -6.20 \mathrm{E}-02 & 1.91 \mathrm{E}-02 & 0.986122 \\ -0.20374 & -2.68 \mathrm{E}-01 & -1.39 \mathrm{E}-01 & 0 \\ -0.3029 & -3.65 \mathrm{E}-01 & -2.41 \mathrm{E}-01 & 0 \\ -0.0449 & -8.89 \mathrm{E}-02 & -8.54 \mathrm{E}-04 & 0.038662 \\ -0.10877 & -1.49 \mathrm{E}-01 & -6.88 \mathrm{E}-02 & 3.26 \mathrm{E}-13 \\ -0.0667 & -1.10 \mathrm{E}-01 & -2.36 \mathrm{E}-02 & 2.2 \mathrm{E}-06 \\ -0.0838 & -1.21 \mathrm{E}-01 & -4.61 \mathrm{E}-02 & 3.42 \mathrm{E}-13 \\ -0.04216 & -9.10 \mathrm{E}-02 & 6.71 \mathrm{E}-03 & 0.23508 \\ -0.15091 & -2.03 \mathrm{E}-01 & -9.89 \mathrm{E}-02 & 2.34 \mathrm{E}-13 \\ -0.10732 & -1.54 \mathrm{E}-01 & -6.03 \mathrm{E}-02 & 2.82 \mathrm{E}-13 \\ -0.12472 & -1.86 \mathrm{E}-01 & -6.33 \mathrm{E}-02 & 7.11 \mathrm{E}-12 \\ -0.14524 & -1.97 \mathrm{E}-01 & -9.30 \mathrm{E}-02 & 3.02 \mathrm{E}-13 \\ -0.27494 & -3.47 \mathrm{E}-01 & -2.03 \mathrm{E}-01 & 0 \\ -0.19397 & -2.53 \mathrm{E}-01 & -1.35 \mathrm{E}-01 & 0\end{array}$

\begin{abstract}
indistinguishable means and red cell indicate means that are
\end{abstract} ANOVA Summary of Finger Type Radial Sections

\begin{tabular}{lrcccc}
\hline & Df & Sum Sq & Mean Sq & $\mathrm{F}$ value & $\operatorname{Pr}(>\mathrm{F})$ \\
\hline Pattern\$Pattern:Pattern\$Radius & 20 & 65 & 3.27 & 26.24 & $<2 \mathrm{e}-16$ \\
Finger\$Finger & 3 & 122 & 40.75 & 322.18 & $<2 \mathrm{e}-16$ \\
Residuals & 45454 & 5673 & 0.12 & & \\
\hline
\end{tabular}

Tukey Test of Different Finger Radius Sections

\begin{tabular}{lllll}
\hline Finger:Radius Comparison & diff & lwr & upr & adj \\
Middle:Radius 001-Index:Radius 001 & -0.04749 & $-1.92 \mathrm{E}-01$ & $9.70 \mathrm{E}-02$ & $1.00 \mathrm{E}+00$ \\
Thumb:Radius 001-Index:Radius 001 & 0.00228 & $-9.98 \mathrm{E}-02$ & $1.04 \mathrm{E}-01$ & $1.00 \mathrm{E}+00$ \\
Middle:Radius 1-Index:Radius 1 & -0.03129 & $-1.32 \mathrm{E}-01$ & $6.92 \mathrm{E}-02$ & $1.00 \mathrm{E}+00$ \\
Ring:Radius 1-Index:Radius 1 & -0.04699 & $-2.16 \mathrm{E}-01$ & $1.22 \mathrm{E}-01$ & $1.00 \mathrm{E}+00$ \\
Thumb:Radius 1-Index:Radius 1 & 0.020588 & $-5.08 \mathrm{E}-02$ & $9.20 \mathrm{E}-02$ & $1.00 \mathrm{E}+00$ \\
Ring:Radius 1-Middle:Radius 1 & -0.0157 & $-2.09 \mathrm{E}-01$ & $1.77 \mathrm{E}-01$ & $1.00 \mathrm{E}+00$ \\
Thumb:Radius 1-Ring:Radius 1 & 0.067578 & $-1.12 \mathrm{E}-01$ & $2.47 \mathrm{E}-01$ & $1.00 \mathrm{E}+00$ \\
Middle:Radius 2-Index:Radius 2 & 0.050971 & $-4.97 \mathrm{E}-02$ & $1.52 \mathrm{E}-01$ & $9.98 \mathrm{E}-01$ \\
Ring:Radius 2-Index:Radius 2 & -0.05296 & $-1.92 \mathrm{E}-01$ & $8.63 \mathrm{E}-02$ & $1.00 \mathrm{E}+00$ \\
Thumb:Radius 2-Index:Radius 2 & 0.034965 & $-2.47 \mathrm{E}-02$ & $9.47 \mathrm{E}-02$ & $9.73 \mathrm{E}-01$ \\
Ring:Radius 2-Middle:Radius 2 & -0.10393 & $-2.71 \mathrm{E}-01$ & $6.34 \mathrm{E}-02$ & $9.37 \mathrm{E}-01$ \\
Thumb:Radius 2-Middle:Radius 2 & -0.01601 & $-1.26 \mathrm{E}-01$ & $9.43 \mathrm{E}-02$ & $1.00 \mathrm{E}+00$ \\
Thumb:Radius 2-Ring:Radius 2 & 0.087926 & $-5.85 \mathrm{E}-02$ & $2.34 \mathrm{E}-01$ & $9.60 \mathrm{E}-01$ \\
Middle:Radius 3-Index:Radius 3 & 0.0274 & $-3.99 \mathrm{E}-02$ & $9.47 \mathrm{E}-02$ & $1.00 \mathrm{E}+00$ \\
Ring:Radius 3-Index:Radius 3 & -0.08221 & $-2.21 \mathrm{E}-01$ & $5.62 \mathrm{E}-02$ & $9.66 \mathrm{E}-01$ \\
Ring:Radius 3-Middle:Radius 3 & -0.10961 & $-2.60 \mathrm{E}-01$ & $4.08 \mathrm{E}-02$ & $6.82 \mathrm{E}-01$ \\
Thumb:Radius 3-Middle:Radius 3 & 0.024196 & $-5.17 \mathrm{E}-02$ & $1.00 \mathrm{E}-01$ & $1.00 \mathrm{E}+00$ \\
Middle:Radius 4-Index:Radius 4 & -0.02797 & $-8.53 \mathrm{E}-02$ & $2.93 \mathrm{E}-02$ & $9.99 \mathrm{E}-01$ \\
Thumb:Radius 4-Index:Radius 4 & 0.02863 & $-1.95 \mathrm{E}-02$ & $7.68 \mathrm{E}-02$ & $9.66 \mathrm{E}-01$ \\
Thumb:Radius 4-Middle:Radius 4 & 0.056597 & $-1.14 \mathrm{E}-02$ & $1.25 \mathrm{E}-01$ & $3.39 \mathrm{E}-01$ \\
Thumb:Radius 5-Index:Radius 5 & 0.011406 & $-3.55 \mathrm{E}-02$ & $5.83 \mathrm{E}-02$ & $1.00 \mathrm{E}+00$ \\
Middle:Radius 6-Index:Radius 6 & 0.006443 & $-7.01 \mathrm{E}-02$ & $8.29 \mathrm{E}-02$ & $1.00 \mathrm{E}+00$ \\
Ring:Radius 6-Index:Radius 6 & -0.10711 & $-3.17 \mathrm{E}-01$ & $1.03 \mathrm{E}-01$ & $9.98 \mathrm{E}-01$ \\
Ring:Radius 6-Middle:Radius 6 & -0.11355 & $-3.34 \mathrm{E}-01$ & $1.07 \mathrm{E}-01$ & $9.97 \mathrm{E}-01$ \\
Thumb:Radius 6-Middle:Radius 6 & 0.044563 & $-3.87 \mathrm{E}-02$ & $1.28 \mathrm{E}-01$ & $9.94 \mathrm{E}-01$ \\
& & & &
\end{tabular}


Thumb:Radius 6-Ring:Radius 6 Middle:Radius 7-Index:Radius 7 Thumb:Radius 7-Index:Radius 7 Thumb:Radius 7-Middle:Radius 7 Middle:Radius 8-Index:Radius 8 Thumb:Radius 8-Index:Radius 8 Thumb:Radius 8-Middle:Radius 8 Thumb:Radius 9-Index:Radius 9 Thumb:Radius 001-Middle:Radius 001

Thumb:Radius 1-Middle:Radius 1 Ring:Radius 001-Index:Radius 001 Ring:Radius 001-Middle:Radius 001 Thumb:Radius 001-Ring:Radius 001 Middle:Radius 10-Index:Radius 10 Ring:Radius 10-Index:Radius 10 Thumb:Radius 10-Index:Radius 10 Ring:Radius 10-Middle:Radius 10 Thumb:Radius 10-Middle:Radius 10 Thumb:Radius 10-Ring:Radius 10 Thumb:Radius 3-Index:Radius 3 Thumb:Radius 3-Ring:Radius 3 Ring:Radius 4-Index:Radius 4 Ring:Radius 4-Middle:Radius 4 Thumb:Radius 4-Ring:Radius 4 Middle:Radius 5-Index:Radius 5 Ring:Radius 5-Index:Radius 5

Ring:Radius 5-Middle:Radius 5 Thumb:Radius 5-Middle:Radius 5 Thumb:Radius 6-Index:Radius 6 Ring:Radius 7-Index:Radius 7 Ring:Radius 7-Middle:Radius 7 Thumb:Radius 7-Ring:Radius 7 Ring:Radius 8-Index:Radius 8 Ring:Radius 8-Middle:Radius 8 Thumb:Radius 8-Ring:Radius 8 Middle:Radius 9-Index:Radius 9 Ring:Radius 9-Index:Radius 9 Ring:Radius 9-Middle:Radius 9 Thumb:Radius 9-Middle:Radius 9 Thumb:Radius 9-Ring:Radius 9 Thumb:Radius 5-Ring:Radius 5

\begin{tabular}{|c|c|c|c|}
\hline 0.158113 & $-5.41 \mathrm{E}-02$ & $3.70 \mathrm{E}-01$ & $6.28 \mathrm{E}-01$ \\
\hline 0.041691 & $-3.10 \mathrm{E}-02$ & $1.14 \mathrm{E}-01$ & $9.80 \mathrm{E}-01$ \\
\hline 0.038691 & $-1.35 \mathrm{E}-02$ & 9.09E-02 & 6.39E-01 \\
\hline-0.003 & $-8.56 \mathrm{E}-02$ & $7.96 \mathrm{E}-02$ & $1.00 \mathrm{E}+00$ \\
\hline 0.038442 & $-6.60 \mathrm{E}-02$ & $1.43 \mathrm{E}-01$ & $1.00 \mathrm{E}+00$ \\
\hline 0.009647 & $-4.44 \mathrm{E}-02$ & $6.37 \mathrm{E}-02$ & $1.00 \mathrm{E}+00$ \\
\hline-0.02879 & $-1.39 \mathrm{E}-01$ & $8.16 \mathrm{E}-02$ & $1.00 \mathrm{E}+00$ \\
\hline 0.031283 & $-2.96 \mathrm{E}-02$ & $9.22 \mathrm{E}-02$ & 9.97E-01 \\
\hline 0.049767 & $-1.14 \mathrm{E}-01$ & $2.14 \mathrm{E}-01$ & $1.00 \mathrm{E}+00$ \\
\hline 0.051874 & $-6.49 \mathrm{E}-02$ & $1.69 \mathrm{E}-01$ & $1.00 \mathrm{E}+00$ \\
\hline NA & NA & NA & NA \\
\hline NA & NA & NA & NA \\
\hline NA & NA & NA & NA \\
\hline-0.27314 & $-3.65 \mathrm{E}-01$ & $-1.81 \mathrm{E}-01$ & $0.00 \mathrm{E}+00$ \\
\hline NA & NA & NA & NA \\
\hline 0.09773 & 4.47E-02 & $1.51 \mathrm{E}-01$ & $4.00 \mathrm{E}-10$ \\
\hline NA & NA & NA & NA \\
\hline 0.370872 & $2.77 \mathrm{E}-01$ & $4.65 \mathrm{E}-01$ & $0.00 \mathrm{E}+00$ \\
\hline NA & NA & NA & NA \\
\hline 0.051596 & 3.89E-03 & 9.93E-02 & $1.48 \mathrm{E}-02$ \\
\hline 0.133807 & $-8.98 \mathrm{E}-03$ & $2.77 \mathrm{E}-01$ & $1.13 \mathrm{E}-01$ \\
\hline-0.14143 & $-2.28 \mathrm{E}-01$ & $-5.44 \mathrm{E}-02$ & $1.56 \mathrm{E}-07$ \\
\hline-0.11347 & $-2.13 \mathrm{E}-01$ & $-1.41 \mathrm{E}-02$ & $5.49 \mathrm{E}-03$ \\
\hline 0.170065 & $7.56 \mathrm{E}-02$ & $2.64 \mathrm{E}-01$ & $1.34 \mathrm{E}-09$ \\
\hline 0.072945 & $1.71 \mathrm{E}-02$ & $1.29 \mathrm{E}-01$ & $2.43 \mathrm{E}-04$ \\
\hline-0.12938 & $-2.18 \mathrm{E}-01$ & $-4.11 \mathrm{E}-02$ & 7.50E-06 \\
\hline-0.20232 & $-3.03 \mathrm{E}-01$ & $-1.02 \mathrm{E}-01$ & $2.49 \mathrm{E}-12$ \\
\hline-0.06154 & $-1.28 \mathrm{E}-01$ & $5.15 \mathrm{E}-03$ & $1.34 \mathrm{E}-01$ \\
\hline 0.051006 & $5.31 \mathrm{E}-03$ & $9.67 \mathrm{E}-02$ & $8.43 \mathrm{E}-03$ \\
\hline NA & NA & NA & NA \\
\hline NA & NA & NA & NA \\
\hline NA & NA & NA & NA \\
\hline NA & NA & NA & NA \\
\hline NA & $\mathrm{NA}$ & NA & NA \\
\hline NA & NA & NA & NA \\
\hline-0.13278 & $-2.54 \mathrm{E}-01$ & $-1.17 \mathrm{E}-02$ & $1.17 \mathrm{E}-02$ \\
\hline NA & NA & NA & NA \\
\hline NA & NA & NA & NA \\
\hline 0.164068 & $3.75 \mathrm{E}-02$ & $2.91 \mathrm{E}-01$ & $3.01 \mathrm{E}-04$ \\
\hline NA & NA & NA & NA \\
\hline 0.140781 & 4.53E-02 & $2.36 \mathrm{E}-01$ & $6.20 \mathrm{E}-06$ \\
\hline
\end{tabular}

***green highlighted cell indicated indistinguishable means and red cell indicate means that are distinguishable

ANOVA Summary of Gender Radial Sections

\begin{tabular}{lccccc}
\hline & Df & Sum Sq & Mean Sq & F value & $\operatorname{Pr}(>F)$ \\
\hline Gender\$Gender:Gender\$Radius & 10 & 19 & 1.88 & 14.735 & $<2 \mathrm{e}-16$ \\
Residuals & 45465 & 5790 & 0.13 & & \\
\hline
\end{tabular}

Tukey Test of Male and Female Radius Sections

\begin{tabular}{lllll}
\hline Gender:Radius Comparison & diff & Iwr & upr & p adj \\
\hline Male:Radius 001-Female:Radius 001 & -0.01489 & $-8.88 \mathrm{E}-02$ & $5.90 \mathrm{E}-02$ & $1.00 \mathrm{E}+00$ \\
Male:Radius 1-Female:Radius 1 & -0.01876 & $-6.42 \mathrm{E}-02$ & $2.67 \mathrm{E}-02$ & $9.98 \mathrm{E}-01$ \\
Male:Radius 2-Female:Radius 2 & 0.004534 & $-4.00 \mathrm{E}-02$ & $4.91 \mathrm{E}-02$ & $1.00 \mathrm{E}+00$ \\
Male:Radius 3-Female:Radius 3 & 0.024007 & $-1.15 \mathrm{E}-02$ & $5.95 \mathrm{E}-02$ & $6.86 \mathrm{E}-01$
\end{tabular}


Male:Radius 8-Female:Radius 8

\begin{tabular}{llll}
-0.02965 & $-7.37 \mathrm{E}-02$ & $1.44 \mathrm{E}-02$ & $6.96 \mathrm{E}-01$ \\
0.00056 & $-5.46 \mathrm{E}-02$ & $5.57 \mathrm{E}-02$ & $1.00 \mathrm{E}+00$ \\
-0.07435 & $-1.23 \mathrm{E}-01$ & $-2.62 \mathrm{E}-02$ & $6.60 \mathrm{E}-06$ \\
-0.04412 & $-7.75 \mathrm{E}-02$ & $-1.07 \mathrm{E}-02$ & $4.49 \mathrm{E}-04$ \\
0.03932 & $6.13 \mathrm{E}-03$ & $7.25 \mathrm{E}-02$ & $4.07 \mathrm{E}-03$ \\
0.069696 & $3.42 \mathrm{E}-02$ & $1.05 \mathrm{E}-01$ & $3.86 \mathrm{E}-10$ \\
-0.03607 & $-7.38 \mathrm{E}-02$ & $1.70 \mathrm{E}-03$ & $8.35 \mathrm{E}-02$ \\
\hline
\end{tabular}

\section{Minutiae Orientation Tukey Test}

Tukey Test of Static and Displaced Minutiae Orientation

\begin{tabular}{lccrr}
\hline Type:Radius Comparison & diff & IWr & upr & p adj \\
\hline Static:Radius 001-Displaced:Radius 001 & -1.56708 & $-4.21 \mathrm{E}+00$ & $1.07 \mathrm{E}+00$ & $8.73 \mathrm{E}-01$ \\
Static:Radius 10-Displaced:Radius 10 & -1.76465 & $-7.71 \mathrm{E}+00$ & $4.19 \mathrm{E}+00$ & $1.00 \mathrm{E}+00$ \\
Static:Radius 2-Displaced:Radius 2 & -0.57757 & $-2.73 \mathrm{E}+00$ & $1.57 \mathrm{E}+00$ & $1.00 \mathrm{E}+00$ \\
Static:Radius 3-Displaced:Radius 3 & -0.6465 & $-2.67 \mathrm{E}+00$ & $1.37 \mathrm{E}+00$ & $1.00 \mathrm{E}+00$ \\
Static:Radius 7-Displaced:Radius 7 & -3.49314 & $-7.84 \mathrm{E}+00$ & $8.58 \mathrm{E}-01$ & $3.35 \mathrm{E}-01$ \\
Static:Radius 9-Displaced:Radius 9 & 2.533694 & $-2.07 \mathrm{E}+00$ & $7.14 \mathrm{E}+00$ & $9.35 \mathrm{E}-01$ \\
Static:Radius 1-Displaced:Radius 1 & -2.10029 & $-4.15 \mathrm{E}+00$ & $-4.93 \mathrm{E}-02$ & $3.74 \mathrm{E}-02$ \\
Static:Radius 4-Displaced:Radius 4 & -2.98949 & $-4.86 \mathrm{E}+00$ & $-1.11 \mathrm{E}+00$ & $2.51 \mathrm{E}-06$ \\
Static:Radius 5-Displaced:Radius 5 & -2.97394 & $-5.17 \mathrm{E}+00$ & $-7.74 \mathrm{E}-01$ & $2.68 \mathrm{E}-04$ \\
Static:Radius 6-Displaced:Radius 6 & -3.92171 & $-7.75 \mathrm{E}+00$ & $-9.70 \mathrm{E}-02$ & $3.68 \mathrm{E}-02$ \\
Static:Radius 8-Displaced:Radius 8 & -3.47976 & $-6.72 \mathrm{E}+00$ & $-2.39 \mathrm{E}-01$ & $1.98 \mathrm{E}-02$ \\
\hline
\end{tabular}




\title{
Appendix E ( $R$ Scripts utilized in the handling and processing of the data collected)
}

\author{
Initial Script For Radius and Section Data
}

setwd("G:/R_Scripts")

\#Load Data===========================================================
Dataset <- read.csv("G:/R_Scripts/Complete_Data_R.csv")
DatasetGood <- Dataset[which(Dataset[,"Quality"] =='Good'),]

\#MANUAL Targetting of radius intervals

Radius $1<-$ subset(DatasetGood, True_r $>.001 \&$ True_r $<=1$ )

Radius $2<$-subset(DatasetGood, True_r $>1 \&$ True_r $<=2$ )

Radius $3<$ - subset(DatasetGood, True_r $>2 \&$ True_r $<=3$ )

Radius 4 <-subset(DatasetGood, True_r $>3 \&$ True_r $<=4$ )

Radius5 <- subset(DatasetGood, True_r $>4 \&$ True_r $<=5$ )

Radius6 <-subset(DatasetGood, True_r $>5 \&$ True_r $<=6$ )

Radius7 <- subset(DatasetGood, True_r $>6 \&$ True_r $r<=7$ )

Radius8 <-subset(DatasetGood, True_r $>7 \&$ True_r $<=8$ )

Radius9 <- subset(DatasetGood, True_r $>8 \&$ True_r $<=9$ )

Radius $10<$-subset(DatasetGood, True_r $>10$ )

\#break down radius into radial angle sections

Radius1a $<-$ subset(DatasetGood, True_r $>.001 \&$ True_r $<=1 \&$ True_Theta $>=0 \&$ True_Theta $<=30$ )

Radius $1 b<-\operatorname{subset}($ DatasetGood, True_r $>.001 \&$ True_r $<=1 \&$ True_Theta $>=30 \&$ True_Theta $<=60$ )

Radius1c<- subset(DatasetGood, True_r $>.001 \&$ True_r $<=1 \&$ True_Theta $>=60 \&$ True_Theta $<=90$ )

Radius1d <-subset(DatasetGood, True_r $>.001 \&$ True_r $<=1 \&$ True_Theta $>=90 \&$ True_Theta $<=120$ )

Radius1e <- subset(DatasetGood, True_r $>.001 \&$ True_r $<=1 \&$ True_Theta $>=1200 \&$ True_Theta $<=150$ )

Radius1f $<$-subset(DatasetGood, True_r $>.001 \&$ True_r $<=1 \&$ True_Theta $>=150 \&$ True_Theta $<=180$ )

Radius1g $\langle-\operatorname{subset}($ DatasetGood, True_r $>.001 \&$ True_r $<=1 \&$ True_Theta $>=180 \&$ True_Theta $\langle=210$ )

Radius1h <-subset(DatasetGood, True_r $>.001 \&$ True_r $<=1 \&$ True_Theta $>=210 \&$ True_Theta $<=240$ )

Radius1i $<-$ subset(DatasetGood, True_r $>.001 \&$ True_r $<=1 \&$ True_Theta $>=240 \&$ True_Theta $<=270$ )

Radius1j <-subset(DatasetGood, True_r $>.001 \&$ True_r $<=1 \&$ True_Theta $>=270 \&$ True_Theta $<=300$ )

Radius1k <-subset(DatasetGood, True_r $>.001 \&$ True_r $<=1 \&$ True_Theta $>=300 \&$ True_Theta $<=330$ )

Radius11 <-subset(DatasetGood, True_r $>.001 \&$ True_r $<=1 \&$ True_Theta $>=330 \&$ True_Theta $<=360$ )

Radius1a $<-$ subset(DatasetGood, True_r $>.001 \&$ True_r $<=1 \&$ True_Theta $>=0 \&$ True_Theta $<=30$ )

Radius $2 \mathrm{a}<-$-subset(DatasetGood, True_r $>1 \&$ True_r $<=2 \&$ True_Theta $>=0 \&$ True_Theta $<=30$ )

Radius3a $<-\operatorname{subset}($ DatasetGood, True_r $>2 \&$ True_r $<=3 \&$ True_Theta $>=0 \&$ True_Theta $<=30$ )

Radius4a $<-$ subset(DatasetGood, True_r $>3 \&$ True_r $<=4 \&$ True_Theta $>=0 \&$ True_Theta $<=30$ )

Radius5a $<-$ subset(DatasetGood, True_r $>4 \&$ True_r $<=5 \&$ True_Theta $>=0 \&$ True_Theta $<=30$ )

Radius6a $<-$ subset(DatasetGood, True_r $>5 \&$ True_r $<=6 \&$ True_Theta $>=0 \&$ True_Theta $<=30$ )

Radius7a <- subset(DatasetGood, True_r $>6 \&$ True_r $<=7 \&$ True_Theta $>=0 \&$ True_Theta $<=30$ )

Radius8a <-subset(DatasetGood, True_r $>7 \&$ True_r $<=8 \&$ True_Theta $>=0 \&$ True_Theta $<=30$ )

Radius9a $<-$ subset(DatasetGood, True_r $>8 \&$ True_r $<=9$ \& True_Theta $>=0 \&$ True_Theta $<=30$ )

Radius10a <-subset(DatasetGood, True_r $>10 \&$ True_Theta $>=0$ \& True_Theta $<=30$ ) 
Radius $7 b<-$ subset(DatasetGood, True_r $>6 \&$ True_r $<=7 \&$ True_Theta $>=30 \&$ True_Theta $<=60$ ) Radius $4 c<-$ subset(DatasetGood, True_r $>3 \&$ True_r $<=4 \&$ True_Theta $>=60 \&$ True_Theta $<=90$ ) Radius2d <-subset(DatasetGood, True_r $>1 \&$ True_r $<=2 \&$ True_Theta $>=90 \&$ True_Theta $<=120$ ) Radius1e <- subset(DatasetGood, True_r $>.001 \&$ True_r $<=1 \&$ True_Theta $>=120 \&$ True_Theta $<=150)$

Radius5f <-subset(DatasetGood, True_r $>4 \&$ True_r $<=5 \&$ True_Theta $>=150 \&$ True_Theta $<=180$ ) Radius9g <- subset(DatasetGood, True_r $>8 \&$ True_r $<=9 \&$ True_Theta $>=180 \&$ True_Theta $<=210$ ) Radius $4 h<-$ subset(DatasetGood, True_r $>3 \&$ True_r $<=4 \&$ True_Theta $>=210 \&$ True_Theta $<=240$ ) Radius2i <- subset(DatasetGood, True_r $>1 \&$ True_r $<=2 \&$ True_Theta $>=240 \&$ True_Theta $<=270$ ) Radius $2 \mathrm{j}<$-subset(DatasetGood, True_r $>10 \&$ True_Theta $>=270 \&$ True_Theta $<=300$ )

Radius10k <-subset(DatasetGood, True_r $>10 \&$ True_Theta $>=300 \&$ True_Theta $<=330$ )

Radius51 <-subset(DatasetGood, True_r $>4 \&$ True_r $<=5 \&$ True_Theta $>=330 \&$ True_Theta $<=360$ )

\#stat calculations

mean(Radius1e[,26])

$\operatorname{var}($ Radius1e[,26])

$\operatorname{sd}($ Radius1e[,26])

$R$ Script of ANOVA and Tukey Analysis of Radius and Angle Sections

setwd("G:/R_Scripts")

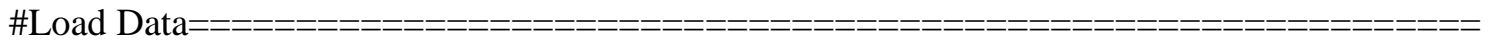

Dataset <- read.csv("G:/R_Scripts/Complete_Data_R.csv")

DatasetGood <- Dataset[which(Dataset[,"Quality"] =='Good'),]

\#\#\#\#Radius ANOVA

radiusANOVA <- read.csv("Radius_ANOVA.csv")

boxplot(radiusANOVA\$Euclidean $\sim$ radiusANOVA $\$$ Radius, notch=TRUE, outline=FALSE, main = "Displacement of Minutiae", ylab="Euclidean (mm)", xlab="Radius")

\#notch places the notches in the sides of the boxes, if the do not overlap the notches of another box then they are likely different means

\#outline=FLASE removes the outlier points from the boxplot

rANOVA <- aov(lm(radiusANOVA $\$$ Euclidean radiusANOVA $\$$ Radius $)$ )

summary(rANOVA)

$\mathrm{x}=$ TukeyHSD(rANOVA)

$\operatorname{plot}(\mathrm{x}$, las $=1)$

\#creates a plot of the Tukey comparisons. Any bar the crosses/contains the line drawn at 0 means the two means are not signigicantly different $=$ they are the same 
$\mathrm{x}[[1]]$

$\mathrm{x} 1=\mathrm{x}[[1]][, 1: 4]$

$\mathrm{x} 2=$ data.frame $(\operatorname{cbind}(\mathrm{x} 1[, 1]$, format $(\mathrm{x} 1[, 2]$, scientific $=\mathrm{TRUE})$, format $(\mathrm{x} 1[, 3]$, scientific $=\mathrm{TRUE})$, format $(\mathrm{x} 1[, 4]$, scientific $=$ TRUE $))$ )

write.csv(x2, "RadiusTukey.csv")

\#\#\#\#angle sections ANOVA

Angle <- read.csv("Angles30.csv")

boxplot(Angle $\$$ Euclidean Angle $\$$ Angle, notch=TRUE, outline=FALSE, main="Displacement of Minutiae", ylab="Euclidean", xlab="Angle Bin")

aANOVA <- aov $(\operatorname{lm}($ Angle\$Euclidean Angle\$Angle $))$

summary(aANOVA)

$\mathrm{y}=$ TukeyHSD(aANOVA)

$\operatorname{plot}(\mathrm{y}$, las $=1)$

$\mathrm{y}[[1]]$

$\mathrm{y} 1=\mathrm{y}[[1]][, 1: 4]$

y2=data.frame (cbind (y1[,1],format(y1[,2], scientific=TRUE), format(y1[,3], scientific=TRUE), format $(\mathrm{y} 1[, 4]$, scientific=TRUE) $))$

write.csv(y2, "Angle30Tukey.csv")

Angle <- read.csv("Angles60.csv")

boxplot(Angle\$Euclidean Angle \$Angle, notch=TRUE, outline=FALSE)

aANOVA <- aov $(\operatorname{lm}($ Angle\$Euclidean Angle\$Angle $))$

summary(aANOVA)

$\mathrm{x}=$ TukeyHSD $($ aANOVA)

$\operatorname{plot}(\mathrm{x}$, las $=1)$

$\mathrm{x}[[1]]$

$\mathrm{x} 1=\mathrm{x}[[1]][, 1: 4]$

$\mathrm{x} 2=$ data.frame $(\operatorname{cbind}(\mathrm{x} 1[, 1]$, format $(\mathrm{x} 1[, 2]$, scientific $=$ TRUE), format $(\mathrm{x} 1[, 3]$, scientific $=$ TRUE), format $(\mathrm{x} 1[, 4]$, scientific $=$ TRUE $))$ )

write.csv(x2, "Angle60Tukey.csv")

Angle <- read.csv("Angles60Offset.csv")

boxplot(Angle\$Euclidean Angle \$Angle, notch=TRUE, outline=FALSE, main="Displacement of Minutiae", ylab="Euclidean", xlab="Angle Bin")

aANOVA $<-\operatorname{aov}(\operatorname{lm}($ Angle\$Euclidean Angle\$Angle $))$

summary(aANOVA) 
$\mathrm{z}=$ TukeyHSD $($ aANOVA)

$\operatorname{plot}(\mathrm{y}, \operatorname{las}=1)$

$\mathrm{z}[[1]]$

$\mathrm{z} 1=\mathrm{z}[[1]][, 1: 4]$

$\mathrm{z} 2=$ data.frame (cbind $(\mathrm{z} 1[, 1]$,format( $\mathrm{z} 1[, 2]$, scientific=TRUE), format(z1[,3], scientific=TRUE),

format $(z 1[, 4]$, scientific $=$ TRUE $))$ )

write.csv(z2, "Angle60OffsetTukey.csv")

Script for Radial, Angular, and Global Euclidean Mean Determination

setwd("G:/R_Scripts")

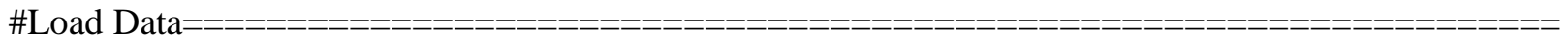

Dataset <- read.csv("Complete_Data_R.csv")

DatasetGood <- Dataset[which(Dataset[,"Quality"] =='Good'), ]

DatasetGood1 <- subset(DatasetGood, True_r > .001)

\#\#\#\#Radii

Radius $1<-$ subset(DatasetGood, True_r $>.001 \&$ True_r $<=1$ )

Radius2 <-subset(DatasetGood, True_r $>1 \&$ True_r $<=2$ )

Radius3 <- subset(DatasetGood, True_r $>2 \&$ True_r $r<=3$ )

Radius4 <-subset(DatasetGood, True_r $>3 \&$ True_r $<=4$ )

Radius 5 <- subset(DatasetGood, True_r $>4$ \& True_r $<=5$ )

Radius6 <-subset(DatasetGood, True_r $>5 \&$ True_r $<=6$ )

Radius7 <- subset(DatasetGood, True_r $>6 \&$ True_r $r=7$ )

Radius $8<$-subset(DatasetGood, True_r $>7 \&$ True_r $<=8$ )

Radius9 <- subset(DatasetGood, True_r $>8 \&$ True_r $<=9$ )

Radius 10<- subset(DatasetGood, True_r $>9$ \& True_r $<=10$ )

Radius $11<-$ subset(DatasetGood, True_r $>10$ )

\#\#\#\#\#Angle Sections

Angle $0<-$ subset (DatasetGood, True_Theta $>=330 \mid$ True_Theta $<30$ )

Angle60 <-subset(DatasetGood, True_Theta > $=30 \&$ True_Theta $<90$ )

Angle120<- subset(DatasetGood, True_Theta >=90 \& True_Theta <150)

Angle $180<$-subset(DatasetGood, True_Theta $>=150 \&$ True_Theta $<=210$ )

Angle $240<-$ subset(DatasetGood, True_Theta $>=210 \&$ True_Theta $<270$ )

Angle300 <-subset(DatasetGood, True_Theta > $=270 \&$ True_Theta <330)

\#\#\#\#Pattern Type

Loops <- DatasetGood1[ which(DatasetGood1[,"Pattern_Type"] == 'Right Slant'),]

Arches <- DatasetGood1[ which(DatasetGood1[,"Pattern_Type"] == 'Plain Arch'), ]

Whorls <- DatasetGood1[ which(DatasetGood1[,"Pattern_Type"] == 'Plain Whorl'),] 
\#\#\#\#-nder

Male <- DatasetGood[ which(DatasetGood[,"Gender"] == 'Male'), ]

Female <- DatasetGood[ which(DatasetGood[,"Gender"] == 'Female'),]

\#\#\#\#\#\#FInger

Index <- DatasetGood[ which(DatasetGood[,"Finger_Number"] == 'Index'),]

Thumb <- DatasetGood[ which(DatasetGood[,"Finger_Number"] == 'Thumb'),]

Middle <- DatasetGood[ which(DatasetGood[,"Finger_Number"] == 'Middle'),]

Ring <- DatasetGood[ which(DatasetGood[,"Finger_Number"] == 'Ring'),]

\#\#\#\#Minutiae Type

EndingRidge <- DatasetGood[ which(DatasetGood[,"Minutiae_Type"] == 'ending ridge'),]

Bifurcation <- DatasetGood[ which(DatasetGood[,"Minutiae_Type"] == 'bifurcation'), ]

\#\#\#\#\#Complete Data Distortions

Right <- DatasetGood1[ which(DatasetGood1[,"Distortion"] == 'Translation Right'),]

Left <- DatasetGood1[ which(DatasetGood1[,"Distortion"] == 'Translation Left'),]

Up <- DatasetGood1[ which(DatasetGood1[,"Distortion"] == 'Translation Up'),]

Down <- DatasetGood1[ which(DatasetGood1[,"Distortion"] == 'Translation Right'), ]

CW <- DatasetGood1[ which(DatasetGood1[,"Distortion"] == 'Torque CW'),]

CCW <- DatasetGood1[ which(DatasetGood1[,"Distortion"] == 'Torque CCW'), ]

Planar <- DatasetGood1[ which(DatasetGood1[,"Distortion"] == 'Planar Glass'),]

\#\#\#\#Arch Distortions

ARight <- Arches[ which(Arches[,"Distortion"] == 'Translation Right'),]

ALeft <- Arches[ which(Arches[,"Distortion"] == 'Translation Left'), ]

AUp <-Arches[ which(Arches[,"Distortion"] == 'Translation Up'), ]

ADown <- Arches[ which(Arches[,"Distortion"] == 'Translation Down'), ]

ACW <- Arches[ which(Arches[,"Distortion"] == 'Torque CW'), ]

ACCW <- Arches[ which(Arches[,"Distortion"] == 'Torque CCW'), ]

APlanar <- Arches[ which(Arches[,"Distortion"] == 'Planar Glass'), ]

\#\#\#\#\#Loops Distortions

LRight <- Loops[ which(Loops[,"Distortion"] == 'Translation Right'),]

LLeft <- Loops[ which(Loops[,"Distortion"] == 'Translation Left'), ]

LUp <- Loops[ which(Loops[,"Distortion"] == 'Translation Up'),]

LDown <- Loops[ which(Loops[,"Distortion"] == 'Translation Down'),]

LCW <- Loops[ which(Loops[,"Distortion"] == 'Torque CW'),]

LCCW <- Loops[ which(Loops[,"Distortion"] == 'Torque CCW'), ]

LPlanar <- Loops[ which(Loops[,"Distortion"] == 'Planar Glass'),]

\#\#\#\#\#Whorl Distortions

WRight <- Whorls[ which(Whorls[,"Distortion"] == 'Translation Right'), ]

WLeft <- Whorls[ which(Whorls[,"Distortion"] == 'Translation Left'),]

WUp <- Whorls[ which(Whorls[,"Distortion"] == 'Translation Up'),]

WDown <- Whorls[ which(Whorls[,"Distortion"] == 'Translation Down'),]

WCW <- Whorls[ which(Whorls[,"Distortion"] == "Torque CW'),]

WCCW <- Whorls[ which(Whorls[,"Distortion"] == 'Torque CCW'), ] 
WPlanar <- Whorls[ which(Whorls[,"Distortion"] == 'Planar Glass'), ]

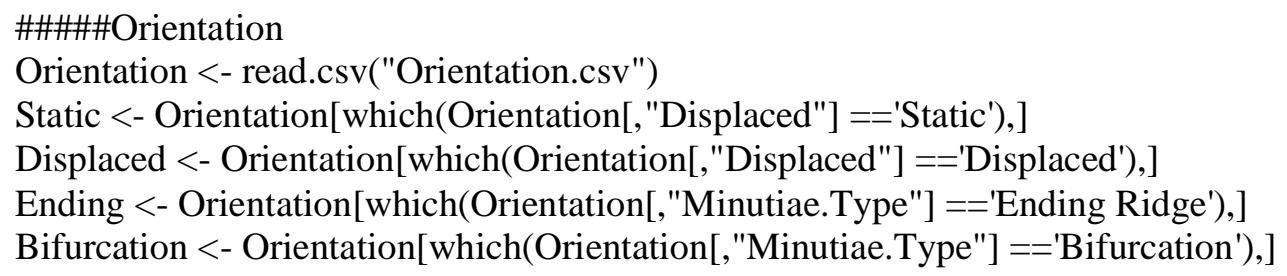

Inked1 =data.frame(cbind(New[,1],format(New[,2]),format(New[,3]))) write.table(Inked1, "InkedRadius.txt", sep="\t", quote = FALSE, row.name=FALSE, col.name=FALSE)

\#COmplete Data Angles

New $=$ matrix $(9999$, nrow $=6$, ncol $=3)$

$\mathrm{k}=1$

for (i in angle)

$\mathrm{k}=\mathrm{k}$ 
testset=subset(Inked, True_Theta $>\mathrm{i} \&$ True_Theta $<=\mathrm{i}+60)$

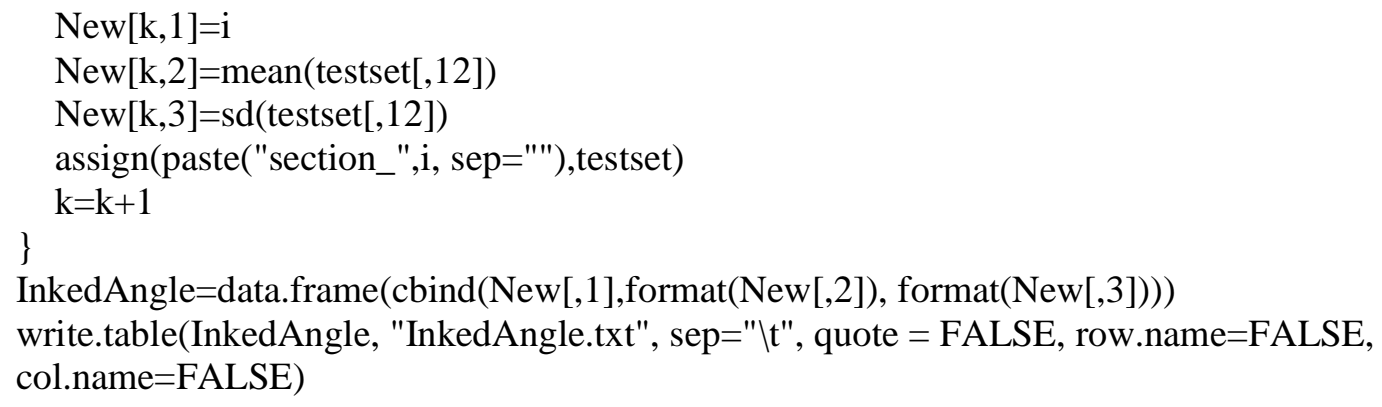




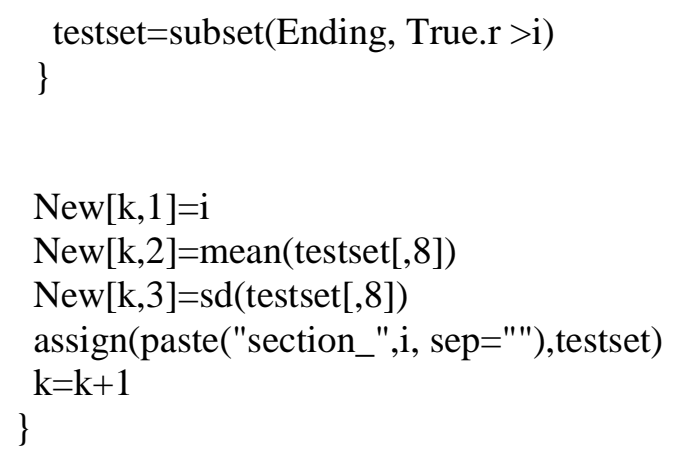

Ending1=data.frame(cbind(New[,1],format(New[,2]),format(New[,3]))) write.table(Ending1, "EndingRadius.txt", sep="ไt", quote = FALSE, row.name=FALSE, col.name=FALSE)

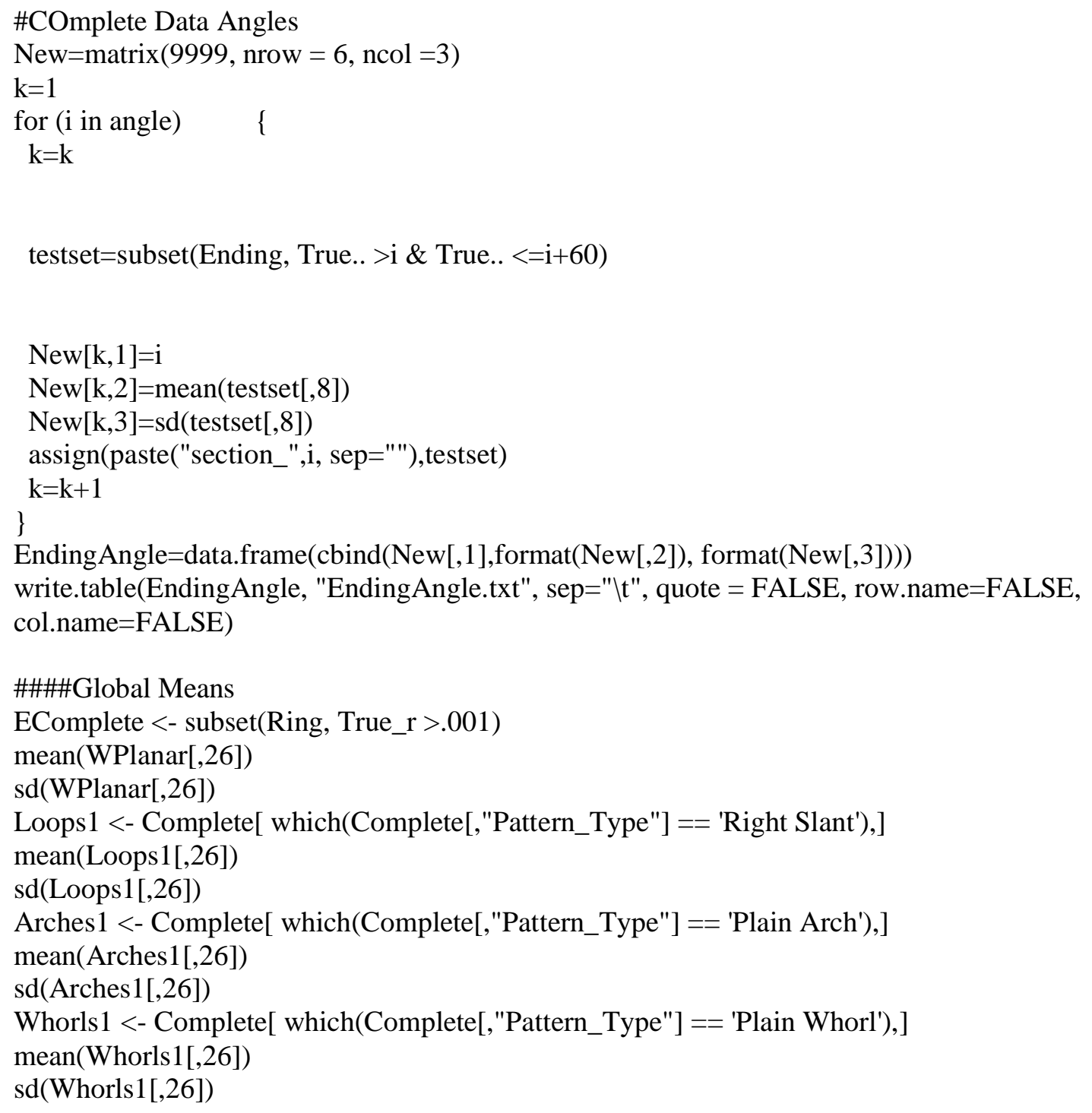


$R$ Script for Calculating Mean Values for Each of the 66 Sections

setwd("G:/R_Scripts")

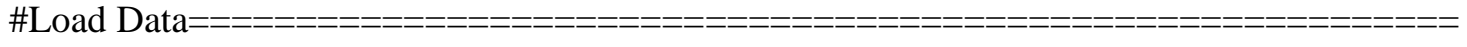

Dataset <- read.csv("G:/R_Scripts/Complete_Data_R.csv")

DatasetGood <- Dataset[which(Dataset[,"Quality"] =='Good'),]

\#contains only the minutiae of quality = good

\#subset based on distortion

Right <- DatasetGood[ which(DatasetGood[,"Distortion"] == 'Translation Right'),]

Left <- DatasetGood[ which(DatasetGood[,"Distortion"] == 'Translation Left'), ]

Up <- DatasetGood[ which(DatasetGood[,"Distortion"] == 'Translation Up'),]

Down <- DatasetGood[ which(DatasetGood[,"Distortion"] == 'Translation Right'),]

CW <- DatasetGood[ which(DatasetGood[,"Distortion"] == 'Torque CW'),]

CCW <- DatasetGood[ which(DatasetGood[,"Distortion"] == 'Torque CCW'), ]

Planar <- DatasetGood[ which(DatasetGood[,"Distortion"] == 'Planar Glass'),]

radius $=\mathrm{c}(.001,1,2,3,4,5,6,7,8,9,10)$

angle $=c(30,90,150,210,270,330)$

$\# \mathrm{i}$ assigned to radius values

$\# \mathrm{j}$ assigned to angle values

section $=\operatorname{rep}(\mathrm{NA}, 11)$

\#\#\#\#\#Section Calculation Loop

New $=$ matrix $(9999$, nrow $=66$, ncol $=4)$

$\mathrm{k}=1$

for ( $\mathrm{i}$ in radius) $\quad\{$

for $(j$ in angle $) \quad\{$

$\mathrm{k}=\mathrm{k}$

if $(\mathrm{i}<10)\{$

testset=subset(DatasetGood, True_r $>$ i \& True_r $<=i+1 \&$ True_Theta $>$ j $\&$ True_Theta $<=j+60$ ) \} else \{ testset=subset(DatasetGood, True_r $>$ i \& True_Theta $>$ j \& True_Theta $<=j+60)$

\}

\#meanval $[\mathrm{i}, \mathrm{j}]=$ mean(testset)

\#sdval $[\mathrm{i}, \mathrm{j}]=\operatorname{sd}($ testset $)$

\#print(mean(testset[,26]))

\#print(sd(testset[,26])) 


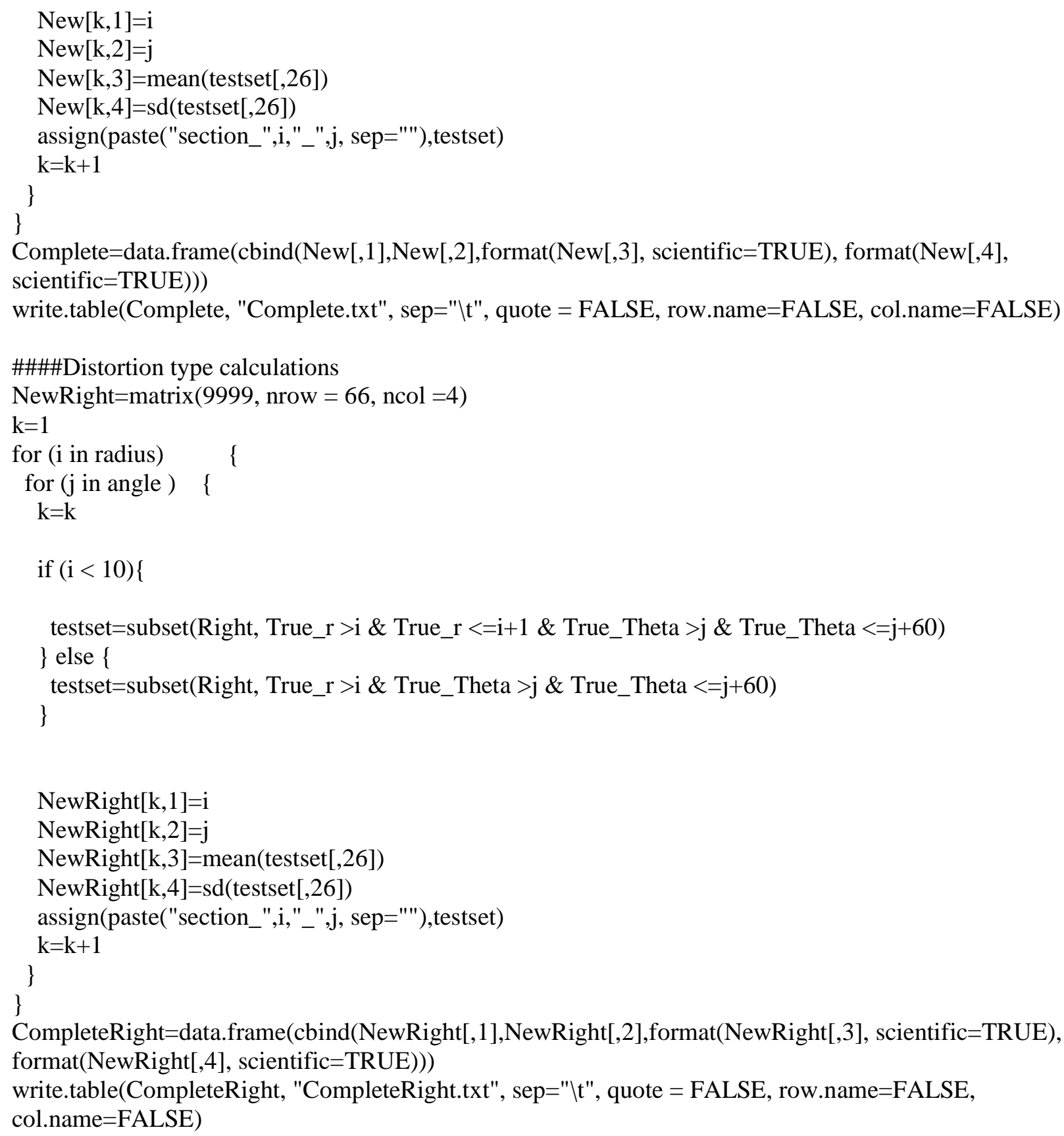

General R Script for the Construction of Variability Ellipse Charts

\#Load Data======================================================

Dataset <- read.csv("G:/R_Scripts/Complete_Data_R.csv") 


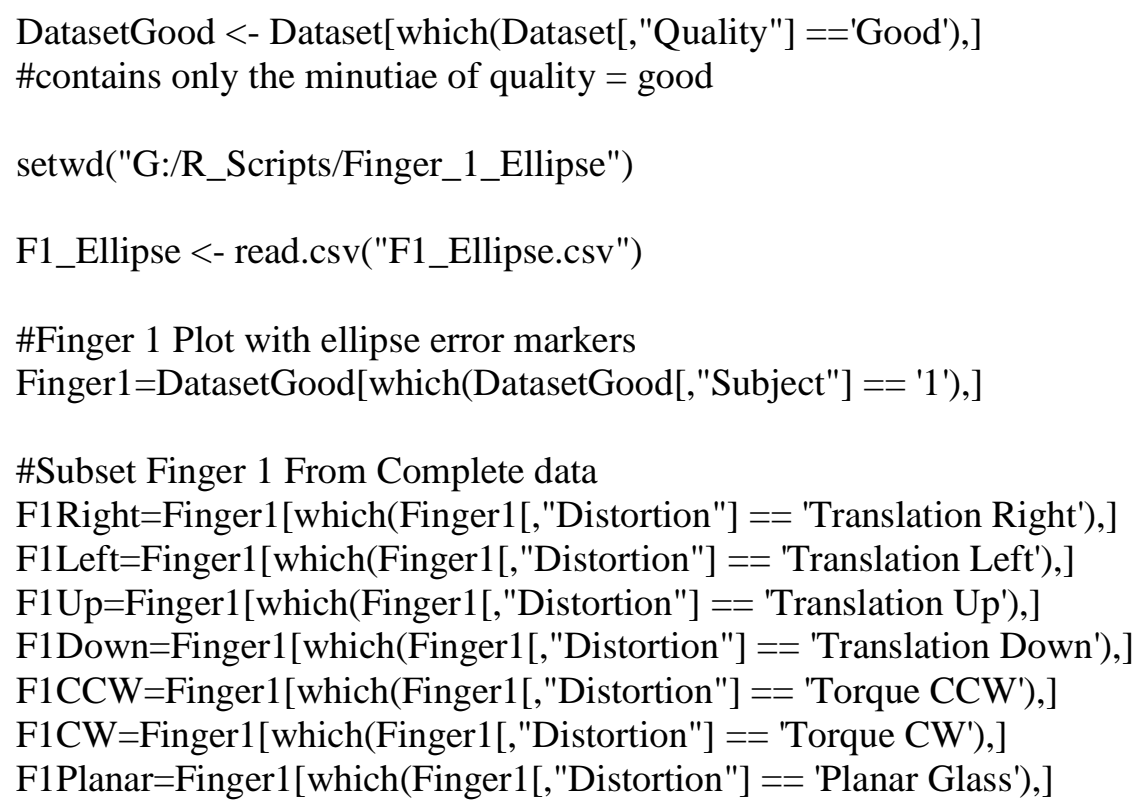




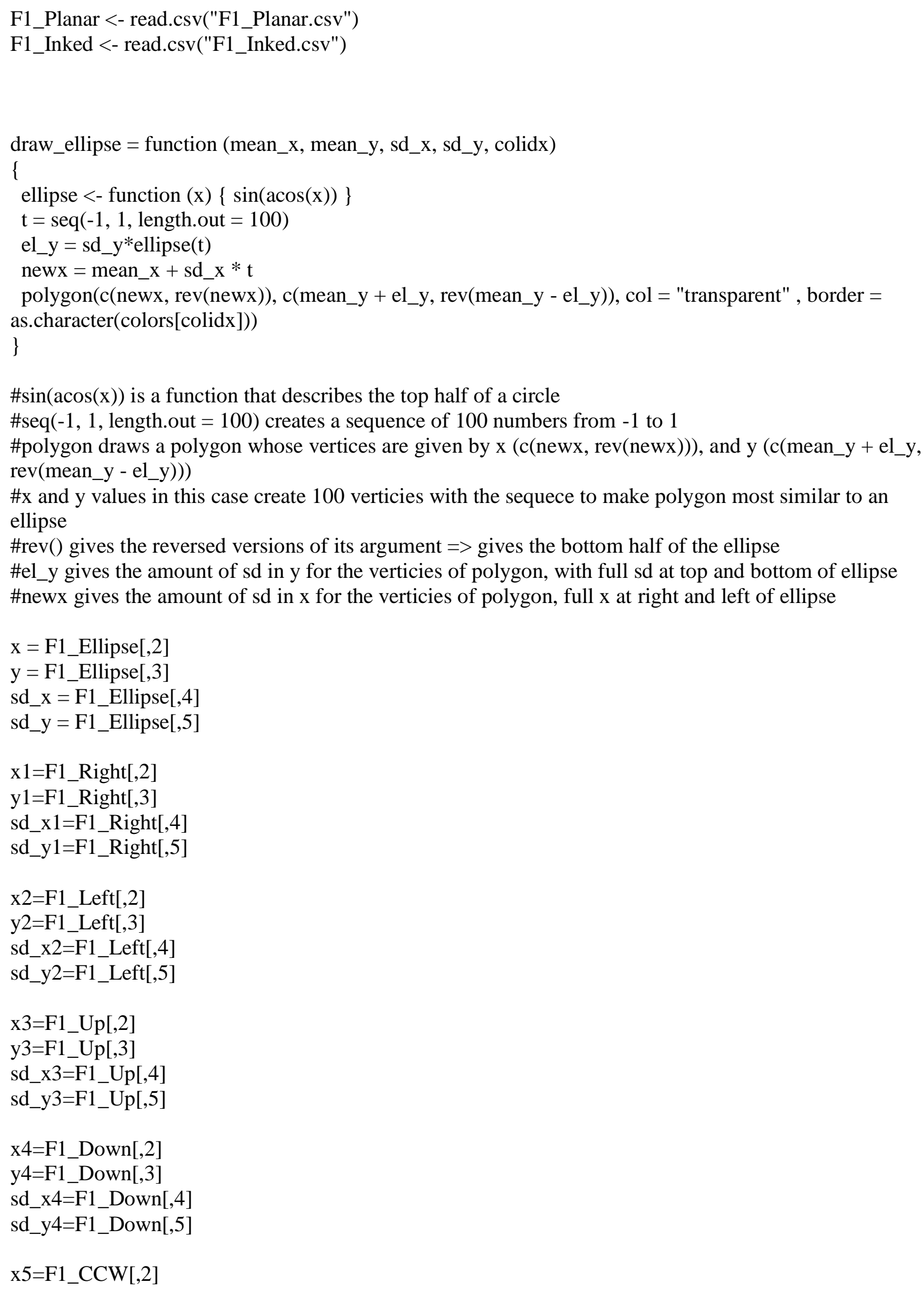




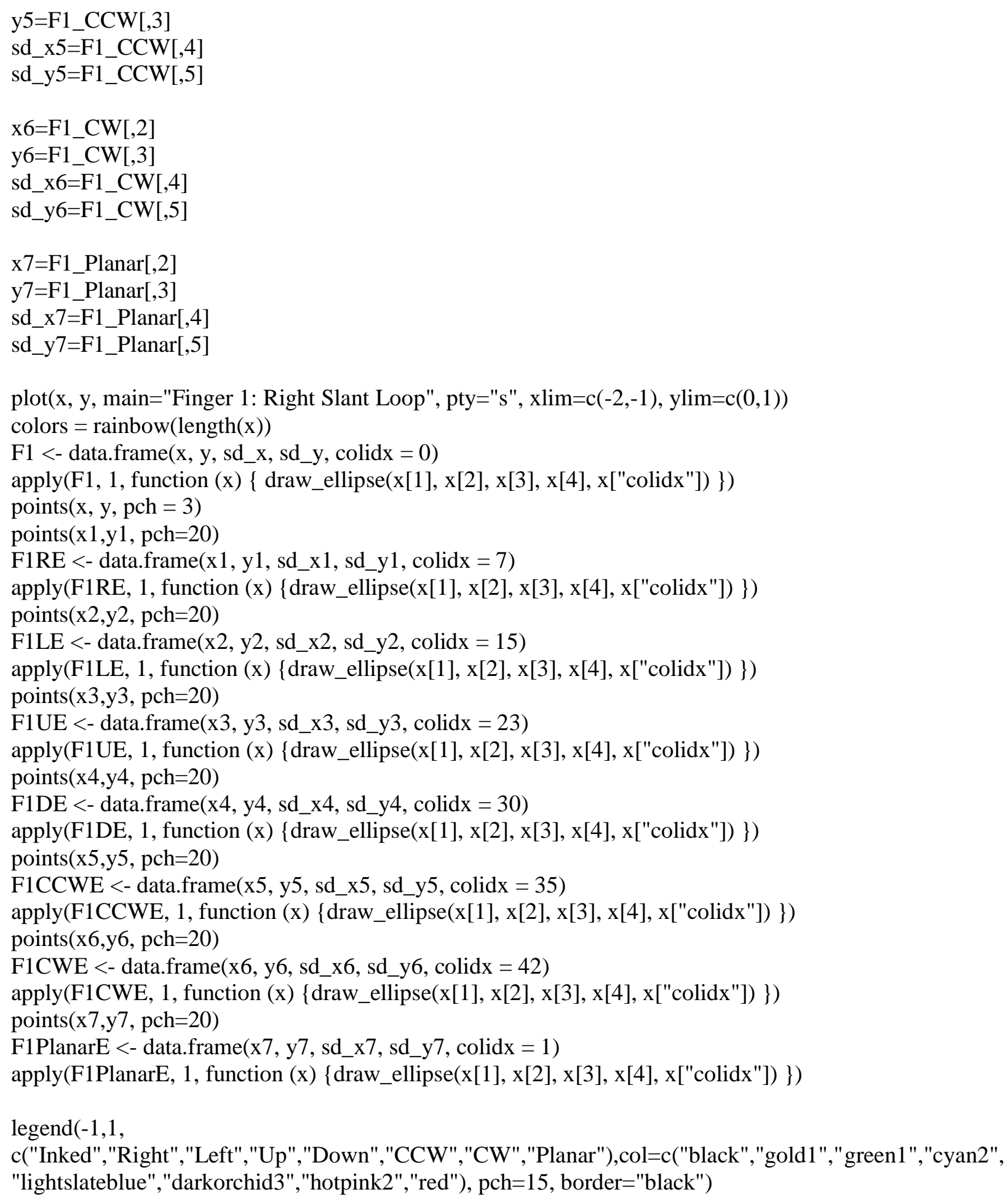


Complete ANOVA and Tukey R Script

setwd("G:/R_Scripts")

\#Load Data====================================================="

Dataset <- read.csv("G:/R_Scripts/Complete_Data_R.csv")

DatasetGood <- Dataset[which(Dataset[,"Quality"] =='Good'),]

\#\#\#\#Radius ANOVA

radiusANOVA <- read.csv("Radius_ANOVA.csv")

boxplot(radiusANOVA $\$$ Euclidean $\sim$ radiusANOVA $\$$ Radius, notch=TRUE, outline=FALSE, main $=$ "Displacement of Minutiae", ylab="Euclidean (mm)", xlab="Radius")

\#notch places the notches in the sides of the boxes, if the do not overlap the notches of another box then they are likely different means

\#outline=FLASE removes the outlier points from the boxplot

rANOVA <- aov(lm(radiusANOVA\$Euclidean radiusANOVA\$Radius)

summary(rANOVA)

$\mathrm{x}=$ TukeyHSD(rANOVA)

$\operatorname{plot}(\mathrm{x}$, las $=1)$

\#creates a plot of the Tukey comparisons. Any bar the crosses/contains the line drawn at 0 means the two means are not signigicantly different $=$ they are the same

$\mathrm{x}[[1]]$

$\mathrm{x} 1=\mathrm{x}[[1]][, 1: 4]$

$\mathrm{x} 2=$ data.frame $(\operatorname{cbind}(\mathrm{x} 1[, 1]$, format $(\mathrm{x} 1[, 2]$, scientific=TRUE), format $(\mathrm{x} 1[, 3]$, scientific=TRUE),

format $(\mathrm{x} 1[, 4]$, scientific=TRUE) $))$

write.csv(x2, "RadiusTukey.csv")

\#\#\#\#angle sections ANOVA

Angle <- read.csv("ANOVA.csv")

boxplot(Angle $\$$ Euclidean $\sim$ Angle $\$$ Angle, notch=TRUE, outline=FALSE, main="Displacement of

Minutiae", xlab="Angle Bin", ylab="Euclidean")

aANOVA $<-\operatorname{aov}(\operatorname{lm}($ Angle $\$$ Euclidean $~$ Angle $\$$ Angle $))$

summary(aANOVA)

$\mathrm{y}=$ TukeyHSD(aANOVA)

$\operatorname{plot}(\mathrm{y}$, las $=1)$

$\mathrm{y}[[1]]$

$\mathrm{y} 1=\mathrm{y}[[1]][, 1: 4]$ 
$\mathrm{y} 2=$ data.frame $(\operatorname{cbind}(\mathrm{y} 1[, 1]$, format $(\mathrm{y} 1[, 2]$, scientific=TRUE), format $(\mathrm{y} 1[, 3]$, scientific=TRUE), format $(\mathrm{y} 1[, 4]$, scientific $=$ TRUE $))$ ) write.csv(y2, "AngleTukey.csv")

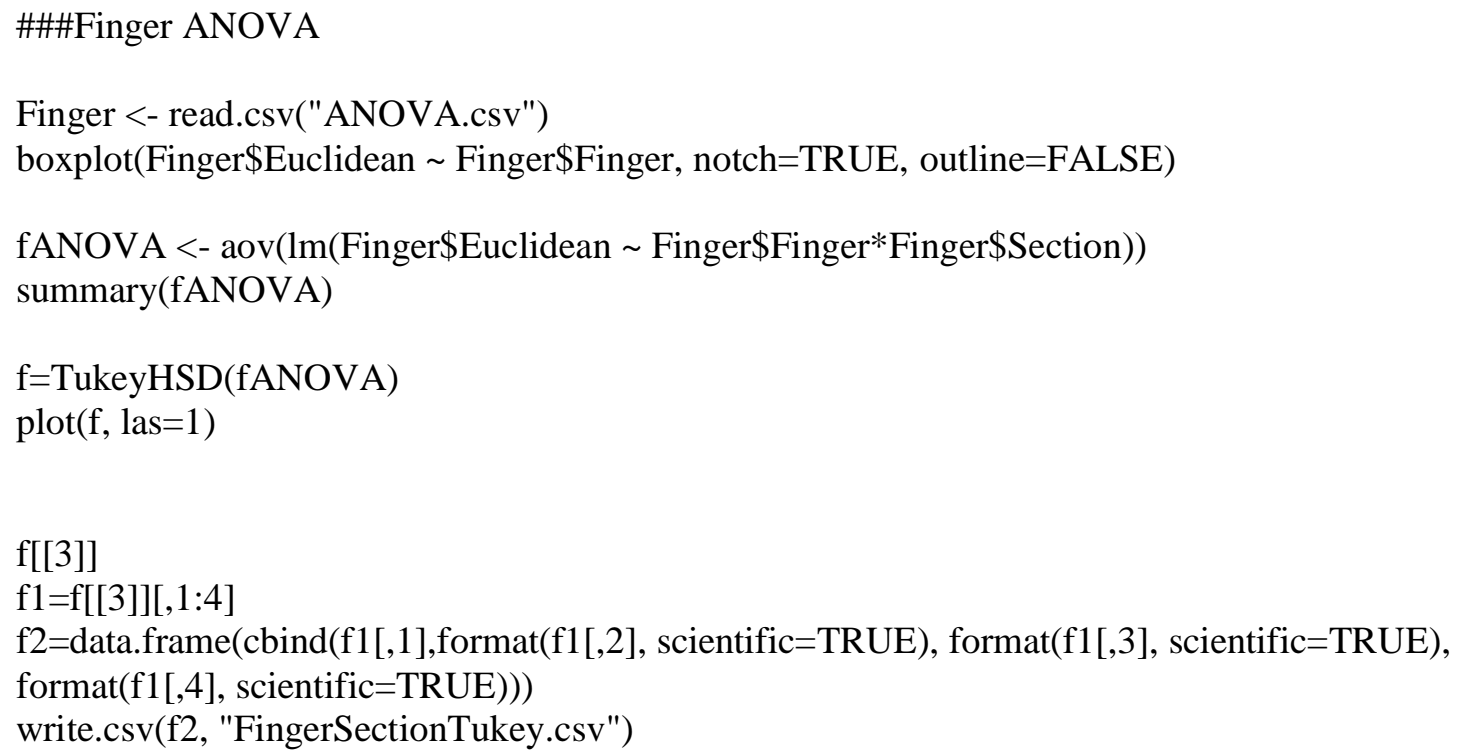

\#\#\#\#ender ANCOVA

Gender <- read.csv("ANOVA.csv")

boxplot(Gender\$Euclidean Gender\$Gender, notch=TRUE, outline=FALSE)

$\mathrm{G}=\operatorname{aov}(\operatorname{lm}($ Gender\$Euclidean $\sim$ Gender\$Gender*Gender\$Radius $))$

summary $(\mathrm{G})$ 
$\mathrm{z}=$ TukeyHSD $(\mathrm{G})$

$\mathrm{z}[[3]]$

$\# \#[[3]]$ targets the third comparison made in the Tukey Test, opposed to [[1]] for first and [[2]] for second

$\mathrm{z} 1=\mathrm{z}[[3]][, 1: 4]$

$\mathrm{z} 2=$ data.frame (cbind $(\mathrm{z} 1[, 1]$,format(z1[,2], scientific=TRUE), format $(\mathrm{z} 1[, 3]$, scientific=TRUE),

format $(z 1[, 4]$, scientific=TRUE) $))$

write.csv(z2, "GenderSectionTukey.csv")

Male <- Gender[which(Gender[,"Gender"] == 'Male'), ]

Female <- Gender[which(Gender[,"Gender"] == 'Female'), ]

qqnorm(Male[,4])

qqline(Male[,4])

\#\#\#\#above vs below core area ANCOVA

$\mathrm{AB}<-$ read.csv("ANOVA.csv")

boxplot $(\mathrm{AB} \$$ Euclidean $\sim \mathrm{AB} \$ \mathrm{AB}$, notch=TRUE, outline=FALSE)

$\mathrm{A}=\operatorname{aov}(\operatorname{lm}(\mathrm{AB} \$$ Euclidean $\sim \mathrm{AB} \$$ Radius*AB $\$ \mathrm{AB}))$

$\operatorname{summary}(\mathrm{A})$

TukeyHSD(A)

\#\#\#\#\#\#Orientation ANOVAS

Orientation <- read.csv("Orientation.csv")

Static <- Orientation[which(Orientation[,"Displaced"] =='Static'),]

Displaced <- Orientation[which(Orientation[,"Displaced"] =='Displaced'),]

Ending <- Orientation[which(Orientation[,"Minutiae.Type"] =-'Ending Ridge'), ]

Bifurcation <- Orientation[which(Orientation[,"Minutiae.Type"] =='Bifurcation'),]

boxplot (Orientation $\$$ Abs $\sim$ Orientation $\$$ Displaced, notch=TRUE, outline=FALSE)

\#notch places the notches in the sides of the boxes, if the do not overlap the notches of another box then they are likely different means

\#outline=FLASE removes the outlier points from the boxplot

\#\#\#\#static vs displaced ANOVA

oANOVA <- aov $(\operatorname{lm}($ Orientation $\$$ Abs Orientation\$Radius $))$

summary(oANOVA)

$\mathrm{o}=$ TukeyHSD(oANOVA)

$\operatorname{plot}(\mathrm{o}$, las $=1)$

\#creates a plot of the Tukey comparisons. Any bar the crosses/contains the line drawn at 0 means the two means are not signigicantly different $=$ they are the same

$\mathrm{o}[[1]]$ 


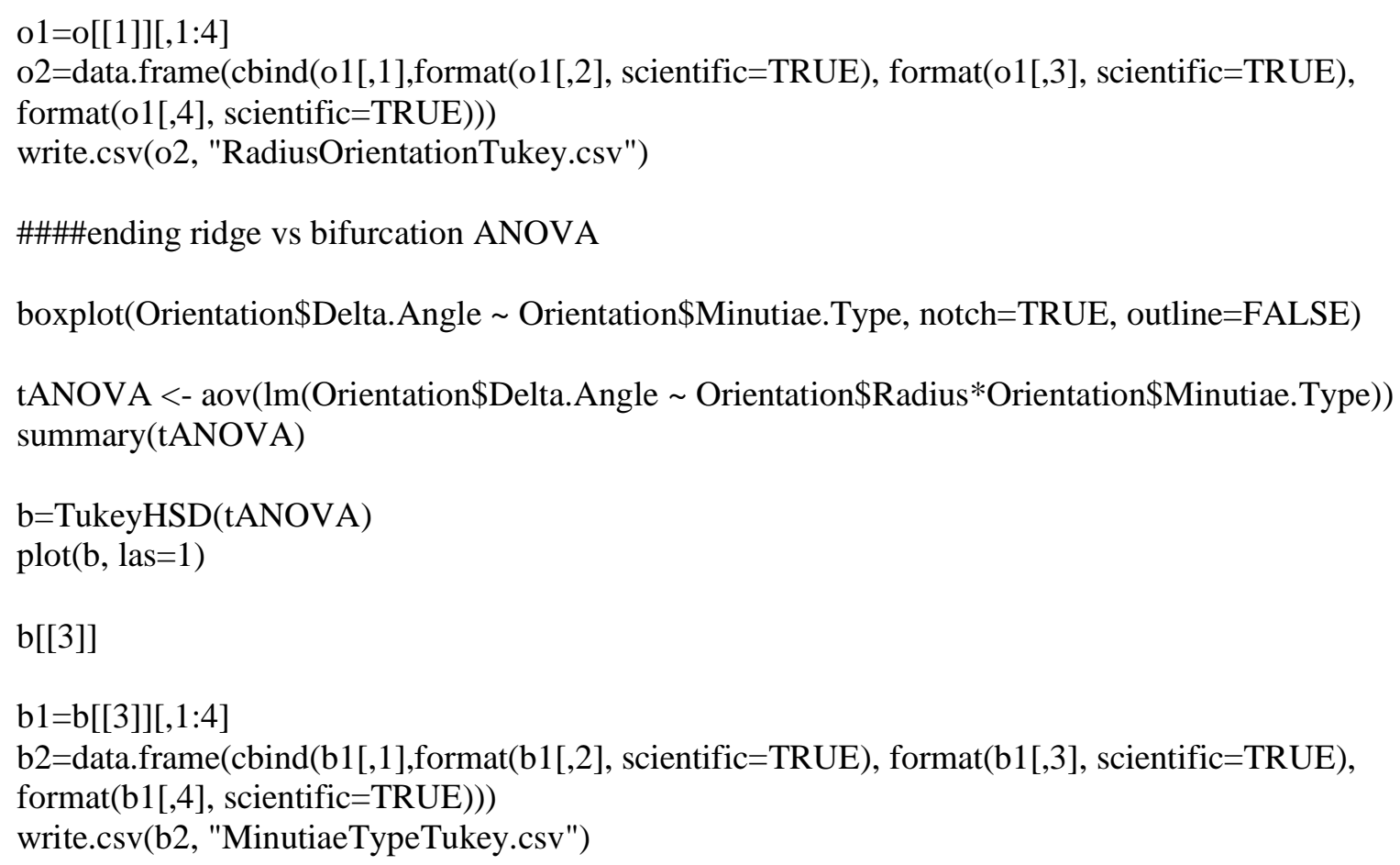

\section{$R$ Script for Polar Plot Grids}

$\operatorname{library}($ plotrix)

$\operatorname{plot}(0,0, p c h=16, p t y=" s ", x \lim =c(-12,12), \operatorname{ylim}=c(-12,12))$

draw.circle $(0,0,1, \mathrm{nv}=100$, border $=$ NULL, $\operatorname{col}=\mathrm{NA}, \mathrm{lty}=1, \mathrm{lwd}=1)$

draw. $\operatorname{circle}(0,0,2, \mathrm{nv}=100$, border $=\mathrm{NULL}, \mathrm{col}=\mathrm{NA}, \mathrm{lty}=1, \mathrm{lwd}=1)$

draw.circle $(0,0,3, \mathrm{nv}=100$, border $=\mathrm{NULL}, \mathrm{col}=\mathrm{NA}, \mathrm{lty}=1, \mathrm{lwd}=1)$

draw $\operatorname{circle}(0,0,4, \mathrm{nv}=100$, border $=\mathrm{NULL}, \mathrm{col}=\mathrm{NA}, \mathrm{lty}=1, \mathrm{lwd}=1)$

draw. $\operatorname{circle}(0,0,5, \mathrm{nv}=100$, border $=\mathrm{NULL}, \mathrm{col}=\mathrm{NA}, 1 \mathrm{ty}=1, \mathrm{lwd}=1)$

draw. circle $(0,0,6, \mathrm{nv}=100$, border $=\mathrm{NULL}, \mathrm{col}=\mathrm{NA}, \mathrm{lty}=1, \mathrm{lwd}=1)$

draw. $\operatorname{circle}(0,0,7, \mathrm{nv}=100$, border $=\mathrm{NULL}, \mathrm{col}=\mathrm{NA}, \mathrm{lty}=1, \mathrm{lwd}=1)$

draw.circle $(0,0,8, \mathrm{nv}=100$, border $=$ NULL, col $=\mathrm{NA}, 1 \mathrm{ty}=1, \mathrm{lwd}=1)$

draw. $\operatorname{circle}(0,0,9, \mathrm{nv}=100$, border $=$ NULL, $\operatorname{col}=\mathrm{NA}, \mathrm{lty}=1, \mathrm{lwd}=1)$

draw.circle $(0,0,10, \mathrm{nv}=100$, border $=$ NULL, col=NA, $1 \mathrm{ty}=1,1 \mathrm{wd}=1)$

draw.circle $(0,0,11, \mathrm{nv}=100$, border $=$ NULL, col=NA, $1 \mathrm{ty}=1,1 \mathrm{wd}=1)$

lines $(\mathrm{c}(0,9.5263), \mathrm{c}(0,5.5))$

lines $(\mathrm{c}(0,0), \mathrm{c}(0,11))$

lines $(\mathrm{c}(0,-9.5263), \mathrm{c}(0,5.5))$

lines $(\mathrm{c}(0,-9.5263), \mathrm{c}(0,-5.5))$

lines $(\mathrm{c}(0,0), \mathrm{c}(0,-11))$

lines $(c(0,9.5263), c(0,-5.5))$

legend(12,12, c("Minimal","Moderate","Significant","Severe"), pch=0, border="black") 